\title{
Causal Order in Parallel and Distributed Simulation Systems
}

\section{Zeng Yi}

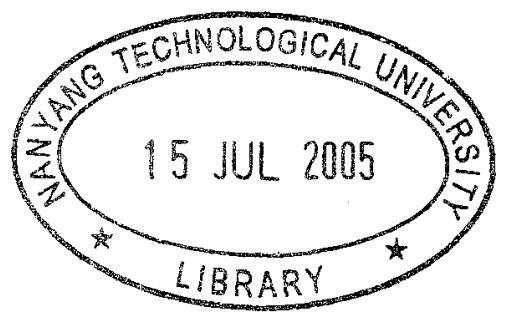

School of Computer Engineering

A thesis submitted to the Nanyang Technological University in fulfilment of the requirement for the Ph.D degree 


\section{Statement of Originality}

I hereby certify that the content of this thesis is the result of work done by myself and has not been submitted for a higher degree to any other University or Institution.

May $/ 27 / 2005$

Date

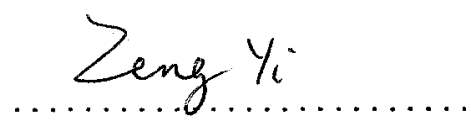

Signature 
To my wife and my dear parents, who always give me love and support. 


\begin{abstract}
The causal order captures the most basic and important event ordering in distributed systems. In our research, it is applied to solve major problems in two categories of parallel and distributed simulation (PADS) applications, i.e., consistency in distributed virtual environments (DVEs) and performance in Time Warp simulation.

In order to achieve more strict consistency in DVEs, inconsistency problems caused by receiving order of concurrent messages are identified. To solve the problems, a causal order based event ordering scheme, the Direct Follow Order (DFO), which enforces uninterrupted delivery of directly coupled causal messages, i.e., direct-follow messages, is proposed. The DFO is proved to be a stricter ordering scheme than the causal order. However, as discovered in our research, the DFO cannot be always ensured. The sufficiency and necessity conditions under which the DFO can be guaranteed on direct-follow messages are derived. Implementation issues of DFO are also discussed.

Performance in Time Warp simulation is greatly determined by the effective advancement of individual logical processes (LPs). Effective advancement can be addressed from various aspects, among which fast cancellation schemes and measures to reduce rollbacks are of our major concerns. These drive our research into two parts: (1)By capturing state dependency and scheduling dependency among events, batch based cancellation scheme is able to carry out rollback optimal cancellation, in which recovery of LPs caused by a straggler message can be done at the cost of at most one rollback; and (2) In view of noteworthy communication latency and possible non-FIFO transmission in distributed simulations, LPs may suffer more rollbacks from their uncontrolled optimistic advancement. To alleviate this problem, the causal order based Time Warp simulation is proposed and relevant techniques making the causal order and the time-stamp order consistent are discussed.
\end{abstract}


Both of the above optimization mechanisms for optimistic PADS are evaluated against traditional Time Warp simulation using popular benchmark models. To be able to conduct fair performance comparison and to provide a high-level abstraction of Time Warp simulation, an extensible framework of Time Warp simulation kernel (TWSK) that is capable of being easily extended for Time Warp executives was also developed. 


\section{Acknowledgement}

I would like to express my deepest gratitude and respect to the following people. Without their help, I could not have done so far.

First and foremost, I would specially thank my supervisor, Dr. Cai Wentong, for his remarkable guidance, heuristic advice and encouragement in my research work. My research work benefits a lot from his enormous supportive efforts, even in writing up of this thesis. Next, I would give my thankfulness to Dr. Stephen John Turner, Dr. Lee Bu Sung and Dr. Zhou Suiping for their patience of listening and discussion with me. Their valuable advices, although critical sometimes, always inspire me. In addition, I also wish to say thanks to Mr. Lu Wei, Mr. Wang Lizhe, Mr. Tang Min, Mr. Zhu Huabing and all others who have supported my research work in one way or another. 


\section{Contents}

$\begin{array}{lll}\text { Abstract } & 1\end{array}$

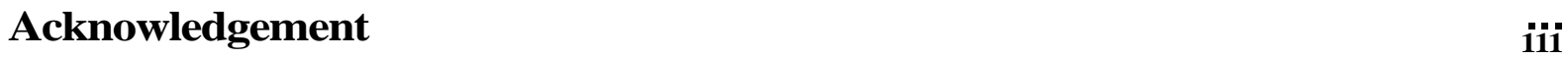

$\begin{array}{lll}\text { Contents } & \text { iv }\end{array}$

$\begin{array}{ll}\text { List of Figures } & \text { ix }\end{array}$

List of Tables $\quad$ xiii

1 Introduction 1

1.1 Overview........................ 1

1.2 Motivations ............................. 4

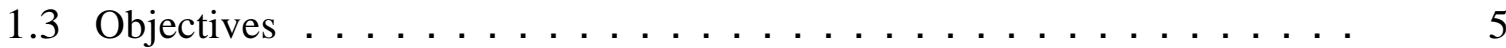

1.4 Approach and Methodology ...................... 7

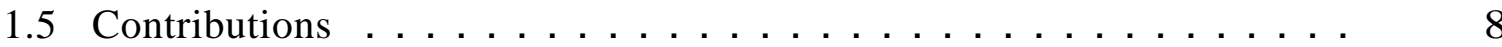

1.6 Synopsis of Thesis. . . . . . . . . . . . . . . . . . 10

2 Literature Review 12 
2.1 The Causal Order . . . . . . . . . . . . . . . . . 12

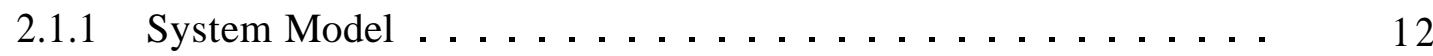

2.1.2 Logical Clocks and Causality $\ldots \ldots \ldots \ldots \ldots \ldots \ldots$

2.1.3 The Causal Order Delivery $\ldots \ldots \ldots \ldots \ldots \ldots$

2.2 Distributed Virtual Environments $\ldots \ldots \ldots \ldots \ldots \ldots$

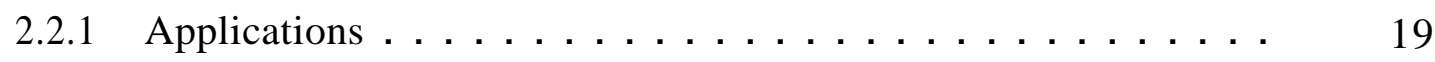

2.2.2 Distributed Systems Support $\ldots \ldots \ldots \ldots \ldots \ldots \ldots \ldots$

2.2 .3 Consistency Models . . . . . . . . . . . . . . . . . . . 24

2.2.4 Consistency Maintenance of Replicated DVEs in the Discrete Domain $\ldots \ldots \ldots \ldots \ldots \ldots \ldots \ldots \ldots \ldots \ldots \ldots$

2.2.5 Consistency Maintenance of Replicated DVEs in the Continuous Domain $\ldots \ldots \ldots \ldots \ldots \ldots \ldots \ldots \ldots \ldots \ldots \ldots$

2.3 Analytic Parallel and Distributed Simulation . . . . . . . . . . 32

2.3.1 Simulation Principles $\ldots \ldots \ldots \ldots \ldots \ldots \ldots \ldots \ldots$

2.3.2 Conservative Synchronization $\ldots \ldots \ldots \ldots \ldots \ldots \ldots$

2.3.3 Optimistic Synchronization $\ldots \ldots \ldots \ldots \ldots \ldots \ldots \ldots$

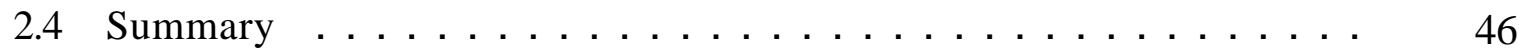

3 Direct-Follow Order Delivery 448

3.1 Introduction and Motivations $\ldots \ldots \ldots \ldots \ldots \ldots \ldots$

3.2 Existing Ordering Schemes $\ldots \ldots \ldots \ldots \ldots \ldots \ldots \ldots$

3.2.1 The Causal Order and Causal Order Delivery $\ldots \ldots \ldots$ 
3.2 .2 Total Order $\ldots \ldots \ldots \ldots \ldots \ldots \ldots \ldots \ldots \ldots \ldots \ldots$

3.2.3 Critical Causality and Causal-Receive Order Delivery . . . . . . 52

3.3 Direct-Follow Relation $\ldots \ldots \ldots \ldots \ldots \ldots \ldots \ldots \ldots$

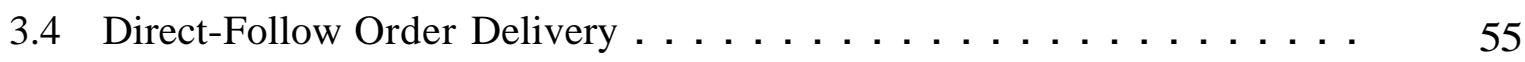

3.5 Resolving Conflicts $\ldots \ldots \ldots \ldots \ldots \ldots \ldots \ldots \ldots$

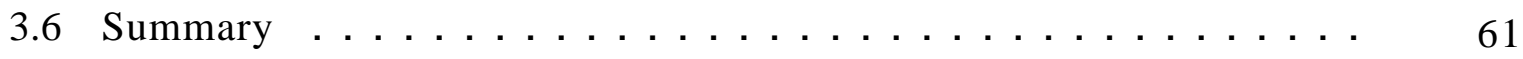

4 TWSK. An Extensible Framework for Time Warp Simulation Kernel 64

4.1 Introduction and Motivations $\ldots \ldots \ldots \ldots \ldots \ldots \ldots \ldots$

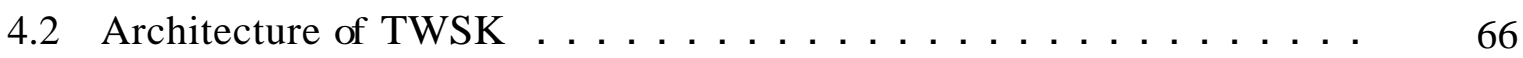

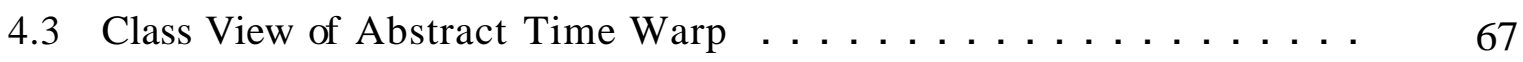

4.3.1 Physical Process $\ldots \ldots \ldots \ldots \ldots \ldots \ldots \ldots$

4.3.2 Logical Process Pool . . . . . . . . . . . . . . 70

4.3.3 Logical Process . . . . . . . . . . . . . . . 70

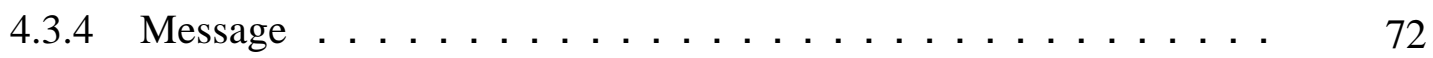

$4.3 .5 \quad$ State $\ldots \ldots \ldots \ldots \ldots \ldots \ldots \ldots \ldots \ldots \ldots \ldots$

4.3.6 Input Queue, Output Queue, State Queue . . . . . . . . 73

4.3.7 Communication Manager and GVT Manager . . . . . . . . 74

4.4 Generalized Mechanisms in Abstract Time Warp . . . . . . . . . . 74

4.4.1 Flow of a Simulation $\ldots \ldots \ldots \ldots \ldots \ldots \ldots$

4.4.2 Running Physical Process $\ldots \ldots \ldots \ldots \ldots \ldots \ldots$

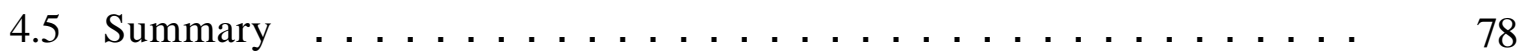


5 Batch Based Cancellation Scheme $\quad 79$

5.1 Introduction . . . . . . . . . . . . . . . . . 79

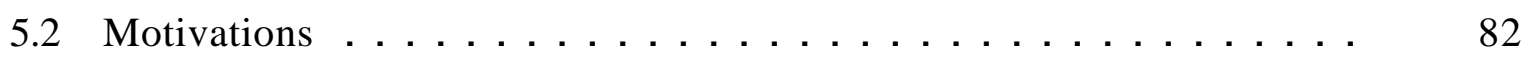

5.3 State Vector . . . . . . . . . . . . . . . 86

5.4 Characterization of Cancelled Events . . . . . . . . . . . . 88

5.5 Batch Based Cancellation Scheme ... . . . . . . . . . . 93

5.5.1 Rollback History $\ldots \ldots \ldots$. . . . . . . . . . . 93

5.5 .2 Implementation . . . . . . . . . . . . . . 95

5.6 Performance Evaluation . . . . . . . . . . . . . . . . . . . . 99

5.6.1 Benchmark Model . . . . . . . . . . . . . . . . . 99

5.6.2 Performance Metrics . . . . . . . . . . . . . . . 100

5.6 .3 Experiments ........................ 102

5.6 .4 Optimizations . . . . . . . . . . . . . . 107

5.7 Summary ............................ 110

6 Causal order based Time Warp 112

6.1 Introduction . . . . . . . . . . . . . . . . . 112

6.2 Motivations ........................... 114

6.3 Effective Path of Advancement . . . . . . . . . . . . 116

6.4 Processing Messages in the Causal Order . . . . . . . . . . . . . . . 117

6.5 Problem of Discrepancies . . . . . . . . . . . . . . . . . 119

6.5.1 Discrepancies between CO and TSO . . . . . . . . . . . 119 
6.5.2 Removing Discrepancies at Sending Side: Reordering . . . . . . . 121

6.5.3 Removing Discrepancies at Receiving Side: Eliminate Reordering 122

6.6 The Causal Order based GVT Approximation . . . . . . . . . . . . 124

6.7 Performance Evaluation . . . . . . . . . . . . . . . 126

6.7.1 Benchmark Model . . . . . . . . . . . . . . 126

6.7.2 Experiments ........................... 128

6.8 Summary . . . . . . . . . . . . . . . . . . . . 135

7 Conclusions and Future Work $\quad 138$

7.1 The Causal Order based Event Ordering in DVEs . . . . . . . . . . 139

7.2 Exploring Causality in Time Warp Simulations . . . . . . . . . . . . 141

Author's Publications

A Sample Code Running on TTW and CTW 


\section{List of Figures}

1.1 Evolution of PADS techniques . . . . . . . . . . . . 3

2.1 A time-space diagram of a distributed computation . . . . . . . . 14

2.2 Data models of DVEs . . . . . . . . . . . . . . . 23

2.3 A scenario of a real-time editing session . . . . . . . . . . . . 27

2.4 Short-term inconsistency and the Local Lag . . . . . . . . . . . . 31

3.1 Inconsistency caused by violation of the causality . . . . . . . . . . 48

3.2 Inconsistency caused by incorrect delivery order of concurrent messages . 49

3.3 Direct-follow messages . . . . . . . . . . . . . 53

3.4 Direct-follow order delivery . . . . . . . . . . . . . 56

3.5 Conflicts in the DFO . . . . . . . . . . . . . . 58

4.1 Two levels of Time Warp simulation . . . . . . . . . . . 64

4.2 An architectural view of TWSK . . . . . . . . . . . . 66

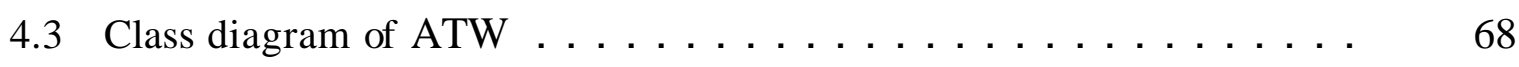

4.4 PhysicalProcess Class and PhysicalProcessInterface Class . . . . . 69

4.5 LogicalProcessPool Class and LogicalProcessPoolInterf ace Class . $\quad 70$ 
4.6 LogicalProcess Class and LogicalProcessInterface Class . . . . . . 71

4.7 Message Class and MessageInterface Class . . . . . . . . . . . . . . 72

4.8 State Class and StateInterface Class . . . . . . . . . . . 73

4.9 InputQueue Class. OutputQueue Class. Statequeue Class and QueueInterface Class . . . . . . . . . . . . . . . . . . . . . 74

4.10 Flow chart of Time Warp simulation . . . . . . . . . . . . 75

4.11 Default implementation of PhysicalProcess : $\operatorname{run}() \ldots \ldots 77$

5.1 Anti-messages chase incorrect computations . . . . . . . . . . 80

5.2 Rollback echoes . . . . . . . . . . . . . . . . . . 81

5.3 Cascading rollbacks . . . . . . . . . . . . . . . 81

5.4 Unnecessary multiple rollbacks . . . . . . . . . . . . . . . 82

5.5 Range based cancellations triggered by a straggler message . . . . . . 85

5.6 Happen-before relation between events . . . . . . . . . . . . . 87

5.7 Propagating and updating rules of state vector at $L P_{i} \ldots \ldots$. . . . . 88

5.8 The rules of event processing at $L P_{i} \ldots \ldots \ldots \ldots \ldots \ldots \ldots$

5.9 The dependence of event e . . . . . . . . . . . . . 92

5.10 Updating rollback history table in FIFO environments . . . . . . . . . . 94

5.11 Processing a scheduled event at $L P_{i} \ldots \ldots \ldots \ldots \ldots \ldots \ldots$

5.12 Scheduling an event at $L P_{i} \ldots \ldots \ldots \ldots \ldots \ldots \ldots$

5.13 Receiving a message from communication channels at $L P_{i} \quad \ldots \ldots 7$

5.14 Rollback to the state prior to executing e at $L P_{i} \ldots \ldots$. . . . . . . 98 
5.15 Batch based cancellations triggered by a straggler message $\ldots . . . .999$

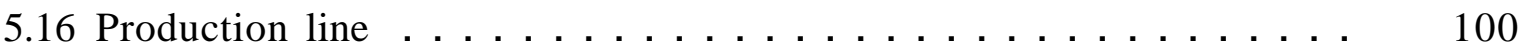

5.17 Efficiency of individual LPs running with fine event granularity _ . . . 104

5.18 Efficiency of individual LPs running with coarse event granularity . . . 104

5.19 Efficiency comparison $\ldots \ldots \ldots \ldots \ldots \ldots$

5.20 Normalized speedup of BTW, BTWopt $\ldots \ldots \ldots \ldots \ldots$

5.21 Production line with extra forward links $\ldots \ldots \ldots \ldots$

6.1 Straggler messages in different environments . . . . . . . . . . 115

6.2 The advancement of LPs . . . . . . . . . . . . . . . 117

6.3 Scheduling an external event by $L P_{i} \ldots \ldots \ldots \ldots \ldots \ldots \ldots \ldots$

6.4 Processing an external message by $L P_{i} \ldots \ldots \ldots \ldots \ldots \ldots \ldots$

6.5 The causal order conflicts with the time-stamp order f . . . . . . 120

6.6 Mattern's GVT approximation . . . . . . . . . . . . . . . . 125

6.7 The causal order based GVT approximation $\ldots \ldots \ldots \ldots \ldots$

6.8 Comparison of Rollback $(L P)$ on cluster (symmetric) _ . . . . . . . . 129

6.9 Comparison of Rollback $(L P)$ in emulated WAN (symmetric) . . . . . 129

6.10 Comparison of Efficiency $(L P)$ (symmetric) . . . . . . . . . 131

6.11 Normalized speedup of CTW (symmetric) $\ldots \ldots \ldots \ldots \ldots$

6.12 Comparison of $\operatorname{Rollback}\left(L P_{i}\right)$ on cluster when GVT=5000 (asymmetric) . 133

6.13 Comparison of Rollback $\left(L P_{i}\right)$ in emulated WAN when GVT=5000 (asym-

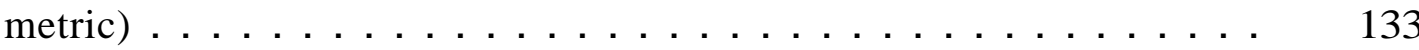


6.14 Comparison of Efficiency worst $(L P)($ asymmetric $) \quad \ldots \ldots \ldots \ldots 134$

6.15 Normalized speedup of CTW (asymmetric) . . . . . . . . 135 


\section{List of Tables}

1.1 Analytic PADS vs. DVEs ..................... 6

2.1 Data models and consistency models in various DVEs . . . . . . . . 25

5.1 Running the simulation with fine event granularity . . . . . . . . 103

5.2 Running the simulation with coarse event granularity . . . . . . . . 103

6.1 Different behaviors between traditional Time Warp and COBTW when handling causally related messages f. . . . . . . . . . . . 123 


\section{Chapter 1}

\section{Introduction}

\subsection{Overview}

The Oxford English Dictionary describes the term "simulation" as:

"The technique of imitating the behavior of some situation or system (economic, mechanical, etc.) by means of an analogous model, situation, or apparatus, either to gain information more conveniently or to train personnel."

Computer simulation represents a broad spectrum of techniques of building a software model of a real or a conceptual system, mimicking its evolvement in designated environments, so that the the simulated system's behavior under specific conditions can be studied.

One classic approach of building computer simulation is referred to as discrete event simulation (DES): The simulated system is expressed in terms of a set of states and events. The evolvement of the system is viewed as the changes of the states, and these changes are discretized at event occurrence instants.

The approach of DES has been adopted for analytic purpose and virtual environment (VE) for many years. Over the past two decades, the rapid expansion of Internet and increasing accessibility to high performance parallel computer has made parallel and 
distributed techniques in DES a hot research field. Tremendous progress in universities and industrial laboratories and the outcome of a wide variety of real-world applications have proved parallel and distributed techniques the most promising solution to strike the performance limitation imposed by the capacity of a single processor.

There are many terms, namely, parallel discrete event simulation (PDES), parallel simulation, distributed simulation or parallel and distributed simulation (PADS), referring to the parallel and/or distributed techniques enabled DES. They generally refer to the same kind of simulation except for their emphasis on different underlying hardware platforms. Parallel simulation is concerned with the execution of simulation programs on tightly coupled multiprocessor computing platforms (e.g., shared-memory multiprocessors, distributed memory multicomputers or an SIMD machines), while distributed simulation is concerned with the execution of simulations on geographically distributed computers interconnected via a local area and/or wide area network, e.g., Internet.

Historically, analytic purpose simulations (analytic PADS) and distributed environments (DVEs) have originated and evolved from three different communities independently (shown in Figure 1.1[108]): Researches in analytic PADS have mainly advanced in the high performance computing community; whereas researches in DVEs have mainly advanced in defense, gaming and Internet communities. These two kinds of simulations eventually have formed the mainstream of parallel and distributed simulation techniques.

Analytic PADS has been successful in the areas of telecommunications [22, 32, 79], digital logic circuits $[15,72,74,77]$, computer architectures $[39,75,76]$ and transportation systems [147]. It has proven to be a cost- and time-effective way for developing new systems, particularly those that involve a high capital investment, and in testing design changes or policy changes to existing systems.

DVEs, however, make their way to provide multi-player enabled distributed interactive environments. Originated from the work of SIMNET [102] developed by DARPA, 


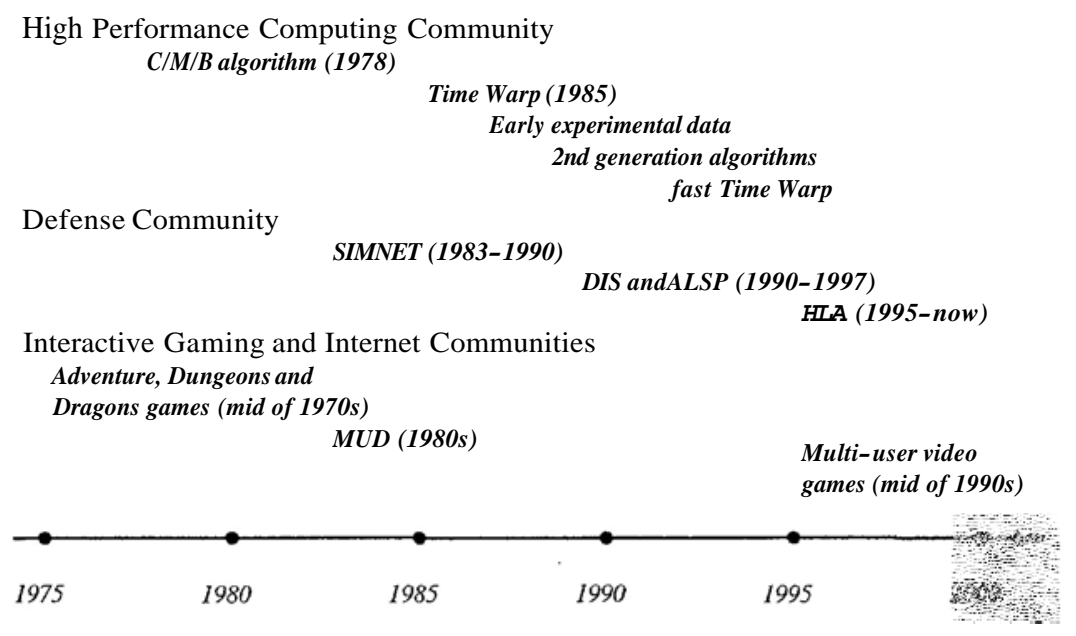

Figure 1.1: Evolution of PADS techniques

standards such as ALSP [148] and DIS [68, 69, 116, 119] and DIS based projects such as NPSNET [90, 92], STOW [37] and PARADISE [67, 128] have been proposed and developed with the aim to architect and build large-scale internetworked simulation environments that would support multi-player interactive 3D-simulations running over a wide-area network and provide low-cost and high-quality battlefield training systems and weapons testing systems. In addition, visual collaborative systems and entertainment systems such as CALVIN [83], DIVE [65],MASSIVE1 [61], MASSIVE2 [62], mWorld [38], Spline [4], REDUCE [133] and GRACE [135] are also developed by researchers.

A major milestone in the evolution of PADS techniques was the standardization of the High Level Architecture (HLA [70]). The HLA is a general-purpose software architecture for simulation reusability and interoperability. From a practical perspective, the HLA defines a standard architecture for all of the modelling and simulation activities in the DoD in the United States. From a technical perspective, it provides a unified architecture for both analytic PADS and DVEs. Prior to the HLA, work in the analytic PADS community and the DVEs community proceeded largely independently of each other. The HLA was a landmark effort in that it began integrating these technologies in a significant way. 


\subsection{Motivations}

Fujirnoto summarized four principal benefits [59] as the reasons to use PADS techniques.

- Reducing execution time (for parallel and distributed simulation). Simulation can be subdivided into computations that run in parallel in multiple processors. The more parallelism explored from the simulation, the less executive time can be achieved.

- Geographical distribution (for distributed simulation). Setup of a simulation on geographically distributed computers enables multiple participants to join from distinct geographical locations. Physically dispersed resources such as specialized equipments, databases can be shared without relocation.

- Integrating simulators that execute on machines from different manufactures (for distributed simulation). Rather than spending efforts to port existing control programs to obtain collaboration, standards for interchanging input/output information and interaction between simulators can be devised to increase reusability of simulators cost-effectively.

- Fault tolerance (for parallel and distributed simulation). Fault tolerance can be achieved by running the simulation on multiple processors. If one processor goes down, it is possible for its backup to carry on the simulation without interruptions.

However, the distributed nature of simulations also gives rise to challenges to PADS techniques. Particularly, different from sequential simulations, a message sent from one processor to another is always experiencing non-zero delay (communication latency). In a parallel computer or a cluster, due to the vicinity of processors and dedicated high speed interconnecting buses/networks, the delay can be as low as a few microseconds to tens of microseconds for a short message containing only a few bytes. While in a 
local area network (LAN) or a wide area network (WAN), the delay can vary from a few milliseconds to seconds.

Despite of the magnitude of communication latency, it is presumable that a global clock or perfectly synchronized local locks are generally not available in a parallel or distributed environment. Thus, simulations have to be synchronized by one means or another.

Over the last two decades, researchers have proposed various synchronization protocols for analytic PADS, which are roughly divided into two categories, namely, conservative synchronization protocols and optimistic synchronization protocols. However, researchers have also shown that, in general, there is no golden rule that either category surpasses the other or which protocol wins over the rest due to a high degree of interweaving of influencing factors. Given the knowledge of a simulation's requirement and communication properties (FIFO, interconnection network topology, abilities to broadcast/multicast), new synchronization protocols or optimizations to the existing ones are still worthy to be studied. Our research work and relevant chapters are briefly summarized in Section 1.5 and Section 1.6. Motivations of each part of the work will be further elaborated at the beginning of the corresponding chapters.

\subsection{Objectives}

Table 1.1 [59] further distinguishes analytic PADS and DVEs from the perspectives of performance and consistency. Their different simulation purposes have resulted in their different requirements for the underlying synchronization protocols.

Analytic PADS typically concerns processing quantitative data of the systems being simulated. For analytic purpose, the simulation is generally running in a non-interrupted way without human's interaction. It is required that, on one hand, the simulation should 
Analytic PADS

Simulation purpose

Human interaction

Performance requirement Consistency requirement quantitative analysis

human is normally an external observer as fast as possible precise simulation and repeatable results
DVEs

create realistic representation of environments

human interacts with environments and controls the embedded entities real-time small inconsistencies are acceptable

Table 1.1: Analytic PADS vs. DVEs

run as fast as possible (optimistic performance) and, on the other hand, the simulation's result must be deterministic and repeatable (strict consistency).

DVEs, however, have the goal to give their users the look and feel of what they could have in the real world. Therefore, the advancement of the simulation is determined by participants' behavior and the simulation should advance in time at approximately the same rate that time advances are perceived by participants in the real world (real-time performance). To achieve this goal, the simulation generally cannot be strictly synchronized. Thus, DVEs have less strict consistency requirement (moderate consistency). During the course of a simulation, inconsistencies are acceptable as long as they are not detrimental to the participants' perceptions. Moreover, the simulation's result is not necessarily required to be repeatable.

In view of these differences, our research sets different objectives for the synchronization protocols for different types of simulations:

- Addressing the consistency issue in DVEs. The level of consistency which could be achieved in DVEs and the characterization of consistency are important research topics. Although there were progresses in recent years, to a large extent the problem of consistency characterization and maintenance still remains unsolved. Only in 
certain areas of DVEs, have significant efforts been made to tackle the problem of consistency.

- Addressing the performance issue of Time Warp simulations. To date, the most elegant approach of optimistic synchronization of analytic PADS is Jefferson's Time Warp and its variants. On one hand, the approach of Time Warp simulation avoids blocking overhead and sensitivity to lookahead, which are regarded as the limitation of conservative synchronization protocols, and exploits more parallelism. On the other hand, it introduces drawbacks known as cascading rollbacks, state saving overhead and demands for larger memory space. Particularly in our research, we aim to improve the performance of Time Warp simulations by the techniques that reduce the number of rollbacks and avoid cascading rollbacks.

\subsection{Approach and Methodology}

The above mentioned research objectives have led our research work to be carried out separately in two directions, i.e., DVEs and Time Warp simulations. However, we address the problems from the same perspective, i.e., event ordering, observing that the study of event ordering is fundamental and critical to most of the distributed systems.

Pioneer work in distributed systems has shown that the happen-before relation, or the causal order (causality) ${ }^{1.1}$, is a fundamental relation universally existing among the events in distributed systems. Mechanisms to capture the causal order and guarantee the delivery of causally related events accordingly (the causal order delivery) have been widely studied as well. Since a simulation, either an analytic PADS or a DVE, is unexceptionally a particular type of distributed systems, the causal order among the events still holds.

In-depth understanding of the causal order and insight of PADS techniques suggests

\footnotetext{
${ }^{1.1}$ We prefer to use the term 'the causal order" or "the causality".
} 
that the causal order is a promising technique to address the consistency and performance issues in parallel and distributed simulations and, thus, forms the basis of our research work. In the study of the consistency issue of DVEs and the performance issue in Time Warp simulations, we spent more efforts on the latter.

As mentioned earlier, the performance of an analytic PADS is prone to be influenced by various factors. In order to minimize possible inaccuracies brought by different running platforms, implementation strategies and adopted communication models in performance comparison, we have made an effort to implement a Time Warp simulation framework on our own.

We first selected WARPED version 1.02, a well-known open-sourced parallel and distributed simulation kernel developed at the University of Cincinnati, as the major reference for our development and carefully studied its implementation details ${ }^{1.2}$. Then, we developed an extensible framework of Time Warp simulation kernel (TWSK) with the hope to enable application level simulations to conduct "fair" performance comparisons. On top of the work of TWSK, we further implemented popular benchmarking simulation models, namely, PHOLD model and manufacturing simulation model, as our benchmarking systems to study and evaluate our proposed Time Warp simulation protocols.

\subsection{Contributions}

The contributions of our work are briefly summarized below:

\section{- DVEs:}

\footnotetext{
${ }^{1.2}$ There are reasons we have not built our simulation framework on top of WARPED. At time we began our development, WARPED was making its important shift, a rewrite, from version 1.02 to a much more complicated version 2.0. Only version 1.02 was ready for public to do further development. However, it lacks object-oriented design and code evolvement over years would have made our development extremely troublesome.
} 


\section{- The direct-follow relation and direct-follow order (DFO) delivery}

scheme. Observing that the causal order is only a partial order, which only achieves consistencies among causally related events, the direct-follow relation was proposed to characterize more semantically close messages, namely, directly coupled causal messages. Delivering messages in the direct-follow order proves to be a stronger ordering mechanism than the causal order and can semantically guarantee higher consistencies in DVEs.

- Time Warp simulation:

\section{- An extensible framework of Time Warp simulation kernel (TWSK).}

TWSK was designed in an object-oriented way with the objectives of extensibility and reusability. Currently, the executives with traditional Time Warp mechanism (TTW), batch based cancellation scheme (BTW) and causal order based Time Warp mechanism (CTW) were implemented in TWSK. They provide identical interfaces to its users, i.e., application level simulations. Thus, the same bennchmarking program can run on top of either executive with minimum changes in its code and "fair" comparisons can be carried out.

\section{- A batch based cancellation scheme in traditional Time Warp sim-}

ulations. To address the pitfalls of rollback echoes, chasing hazards and cascading rollbacks, which can be identified as the major reasons for the inefficiency of the conventional per-event based cancellation scheme, a batch based cancellation scheme was proposed with the hope to do fast cancellation. The proposed scheme proves to be rollback optimal, which means that the recovery of a logical process caused by a straggler message can be done at the cost of at most one rollback. This approach is proved to be significant in removing the above pitfalls. 
- The causal order based Time Warp simulation (COBTW). We stepped further to incorporate the causal order delivery into Time Warp simulations. In a distributed simulation, especially the one where logical processes communicate via non-FIFO channels with noteworthy delay and jitter or where multicast messages are prevailing, the causal order delivery is employed to introduce conservatism among the logical processes to reduce the number of straggler messages in the simulation. Empirically results from the experiments running on a cluster and an emulated WAN confirmed potential performance gain of applying COBTW in certain simulations.

\subsection{Synopsis of Thesis}

The remainder of the thesis is structured as follows:

- Chapter 2 presents a general survey of the causal order and the state-of-the-art PADS techniques. We review consistency issues found in DVEs, performance issues found in Time Warp simulations, as well as simulation benchmarks for performance evaluations.

- Chapter 3 discusses several existing ordering schemes for consistency maintenance, and then describes our proposed direct-follow relation and direct-follow order delivery scheme.

- Chapter 4 elaborates the design objectives, the overall architecture of TWSK. The abstraction of Time Warp mechanism results in an abstract Time Warp executive (ATW), which is elaborated in details. ATW is extended in our implementations of an traditional Time Warp (TTW), a TTW with batch based cancellation (BTW) and a causal order based Time Warp (CTW). 
- Chapter 5 presents the rollback optimal batch based cancellation scheme in traditional Time Warp simulations. The formal proof of the correctness of the scheme is given. The performance evaluation is carried out by running manufacturing simulation over the traditional Time Warp kernel. Further optimizations and variants to the scheme are also discussed.

- Chapter 6 explores the techniques to incorporate the causal order into Time Warp simulations and studies its advantage in reducing straggler messages in distributed and/or multicast enabled simulations. The performance evaluation is done by running PHOLD model over the traditional Time Warp kernel and the causal order based Time Warp kernel.

- Chapter 7 concludes the achievement of our research work and suggests possible areas for future work. 


\section{Chapter 2}

\section{Literature Review}

\subsection{The Causal Order}

\subsubsection{System Model}

The causal order is fundamental to many problems, e.g., consistent global snapshot [1, 3, 23, 98], global predicates [33, 94], distributed termination detection [96] and distributed systems observation [126], in distributed systems. A widely accepted model of distributed systems [126] is given as follows:

Definition 2.1.1 (Distributed System). A distributed system consists of $N$ sequential processes $P_{1}, \ldots, P_{N}$, communicating solely by message-passing. These processes do not share common memory. Their local states are assumed to be physically dispersed. The behavior of each process consists of changing local state, sending and receiving messages. The actions taken by a process are completely determined by an algorithm running locally at the process. It is assumed that a message can be sent to an arbitrary set of destinations through transmission channels in one send action (i.e., multicast is assumed). The transmission channels are assumed reliable, but FIFO is not required (i.e., messages may not arrive at their destinations in the order that they were sent). Messages in transmission may suffer from arbitrary non-zero delays. Furthermore, there is no global clock or 
perfectly synchronized local clocks available.

Definition 2.1.2 (Event). In a distributed system, the actions performed by the local algorithm are called events. By Definition 2.1.1, it suffices to categorize three kinds of events, namely send events, receive events and internal events. They represent the actions of sending messages, receiving messages and the local actions within a process respectively. Let $\mathrm{E}_{\mathrm{i}}$ denote the set of events occurring at $P_{i}$, and let $\mathrm{E}=E_{1} \cup \ldots \cup E_{N}$ denote the set of all events in a distributed system. Since each process is assumed sequential, the events in $E_{i}$ are totally ordered by the sequence of their occurrences and can be indexed. Let $\mathrm{E}_{\mathrm{i}}=\left\{e_{i 1}, e_{i 2}, \ldots, e_{i n}, \ldots\right\}$. This indexed enumeration is referred to as the standard enumeration of $\mathrm{E}_{\dot{r}}$

Definition 2.1.3 (The Causal Order). Given the standard enumeration of $E_{i}$, the causal order $\rightarrow \subseteq E \times E$ is the smallest transitive relation satisfying:

1. If $e_{i m}, e_{i n} \in E_{i}$ and $m<n, e_{i m} \rightarrow e_{i n}$;

2. If $e_{i m} \in E_{i}$ is the send event of a message and $e_{j n} \in E j$ is the corresponding receive event, then $e_{i m} \rightarrow e_{j n}$.

Definition 2.1.3 states that the causal order only exists in some, but not necessarily all, pairs of events in a distributed system. Thus, the causal order is a partial order. For any pair of events which have no causal relation, they are defined to be concurrent as shown below.

Definition 2.1.4 (Concurrency). The concurrency relation $\| \subseteq E \times E$ is defined as: for events $e_{1}$ and $e_{2}, e_{1} \| e_{2}$ iff $\neg\left(e_{1} \rightarrow e_{2}\right)$ and $\neg\left(e_{2} \rightarrow e_{1}\right)$.

The causal order and the concurrency relation among events are illustrated in a socalled time-space diagram in Figure 2.1, in which an event is drawn as a solid dot and a 


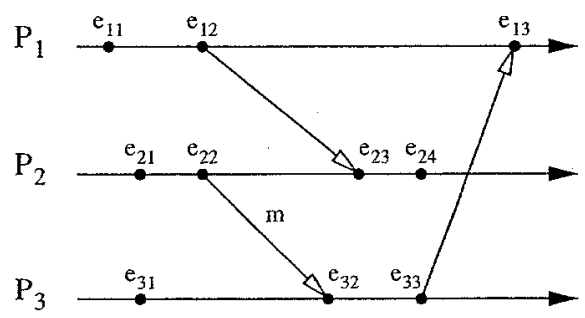

Figure 2.1: A time-space diagram of a distributed computation

message is drawn as a directed arrow. Within each process, events are causally ordered by their indices, e.g., $e_{11} \rightarrow e_{12}$ and $e_{21} \rightarrow e_{24}$. Among the processes, the message passing communication results in several immediate causality, i.e., $e_{12} \rightarrow e_{23}, e_{22} \rightarrow e_{32}$ and $e_{33} \rightarrow e_{13}$. Furthermore, by transitivity, we have $e_{11} \rightarrow e_{24}$ and $e_{21} \rightarrow e_{13}$ as well. There are also concurrent events, for example, $e_{12} \| e_{22}$ and $e_{24} \| e_{33}$, since they cannot be causally ordered in either way.

\subsubsection{Logical Clocks and Causality}

Since there is no perfectly synchronized physical clock available in the system model (Definition 2.1.1), a logical clock is generally used in distributed systems. A logical clock is 'logical"'in the sense that it is based on the order of events instead of physical time.

Let $L C_{i}$ denote the logical clock for process $P_{i}$, which assigns clock value $L C_{i}(e)$ to $e, e \in E_{i}$. Let LC denote the logical clock for the entire system which assigns clock value $\mathrm{LC}(\mathrm{e})$ to e, e $\in E$. $L C(e)=L C_{i}(e), e \in E_{i} \subset E$. Let clock values of all the events in $E$ form a partially ordered set $(\{L C(e) \mid e \in E\},<)$. It is obvious that $L C$ is actually a function with mapping: $E \rightarrow\{L C(e) \mid e \in E\}$.

Regarding a logical clock's ability to express causality, a logical clock can be a weak clock or a strong clock.

Definition 2.1.5 (Weak Clock). An $L C$ is said to be a weak clock or consistent with causality if $\forall e_{1}, e_{2} \in E$ 


$$
e_{1} \rightarrow e_{2} \Rightarrow L C\left(e_{1}\right)<L C\left(e_{2}\right)
$$

Definition 2.1.6 (Strong Clock). An $L G$ is said to be a strong clock or characterizing causality if $\forall e_{1}, e_{2} \in E$

$$
e_{1} \rightarrow e_{2} \Leftrightarrow L C\left(e_{1}\right)<L C\left(e_{2}\right)
$$

Note that even an ideally perfectly synchronized physical clock $P C$ can only be a weak clock because $P C\left(e_{1}\right)<P C\left(e_{2}\right)$ does not imply that $e_{1} \rightarrow e_{2}$.

Weak clocks, e.g., Lamport time [80] and physical clock, are insufficient to characterize causality. Therefore, strong clocks are used in our research to find out the causal relationship among the events.

Causal history $[50,107,113,149]$ is a straightforward scheme that the clock value of event $e$ is by definition a set of events which causally precede $e$.

Definition 2.1.7 (Causal History). Causal history defines a mapping $\mathrm{CH}: \mathrm{E} \rightarrow$ event sets,

1. For the first event $e_{i 1} \in E_{i}$, if it is the corresponding receive event of $e_{j n} \in E_{j}$, $C H\left(e_{i 1}\right)=C H\left(e_{j n}\right) \cup\left\{e_{i 1}\right\}$, else $C H\left(e_{i 1}\right)=\left\{e_{i 1}\right\}$.

2. For other events $e_{i m} \in E_{i}, m>1$, if it is the corresponding receive event of $e_{j n} \in E_{j}$, $C H\left(e_{i m}\right)=C H\left(e_{i(m-1)}\right) \cup C H\left(e_{j n}\right) \cup\left\{e_{i m}\right\}$, else $C H\left(e_{i m}\right)=C H\left(e_{i(m-1)}\right) \cup\left\{e_{i m}\right\} . \sharp$

The binary relation $<$ on $\{C H(e) \mid e \in E\}$ is defined as the subset relation, i.e., for event sets $\mathrm{CH}_{1}, \mathrm{CH}_{2} \in\{\mathrm{CH}(e) \mid e \in E\}, \mathrm{CH}_{1}<\mathrm{CH}_{2}$ means $\mathrm{CH}_{1} \subset \mathrm{CH}_{2}$.

It is easy to prove that causal history is a strong clock. Refer back to Figure 2.1, $C H\left(e_{11}\right)=\left\{e_{11}\right\}, C H\left(e_{12}\right)=\left\{e_{11}, e_{12}\right\}, C H\left(e_{24}\right)=\left\{e_{11}, e_{12}, e_{21}, e_{22}, e_{23}, e_{24}\right\}$ and $C H\left(e_{33}\right)=$ $\left\{e_{21}, e_{22}, e_{31}, e_{32}, e_{33}\right\}$. The causal order among them can be verified by Definition 2.1.7. 
The major problem of causal history is that $C H(e)$ grows unlimitedly as the computation proceeds, which makes it impossible to send this unbounded clock value among the processes. Refinement of causal history forms another kind of clock, vector time [49, $51,97,132]$. Informally, vector time can be regarded as a compressed version of causal history in the sense that it is an N-entry vector whose $k^{t h}, 1 \leq k \leq N$, entry denotes the biggest index of events from $P_{k}$ in causal history.

Definition 2.1.8 (Vector Time). Vector time defines a mapping $V T: E \rightarrow$ vectors,

1. For the first event $e_{i 1} \in E_{i}$, if it is the corresponding receive event of $e_{j n} \in E$, $V T\left(e_{i 1}\right)=V T\left(e_{j n}\right)$ and $V T\left(e_{i 1}\right)[i]=1$, else $V T\left(e_{i 1}\right)[k]=0, k \neq i$ and $V T\left(e_{i 1}\right)[i]=$ 1.

2. For other events $e_{i m} \in E_{i}, m>1$, if it is the corresponding receive event of $e_{j n} \in E_{j}$, $V T\left(e_{i m}\right)=$ Supreme $\left(V T\left(e_{i(m-1)}\right), \operatorname{VT}\left(e_{j n}\right)\right)$ and $V T\left(e_{i m}\right)[i]=V T\left(e_{i(m-1)}\right)[i]+1$, else $V T\left(e_{i m}\right)=V T\left(e_{i(m-1)}\right)$ and $V T\left(e_{i m}\right)[i]=V T\left(e_{i(m-1)}\right)[i]+1$.

The binary relation $<$ on $\{V T(e) \mid e \in E\}$ is defined as: for $V T_{1}, V T_{2} \in\{V T(e) \mid e \in E\}$, $V T_{1}<V T_{2}$ iff $V T_{1}[k] \leq V T_{2}[k], 1 \leq k \leq N$ and $\exists k^{\prime}, V T_{1}\left[k^{\prime}\right]<V T_{2}\left[k^{\prime}\right], 1 \leq k^{\prime} \leq N$. Supreme $\left(V T_{1}, V T_{2}\right)$ constructs a new vector $\left.V T_{0}, V T_{0}[k]=\operatorname{Max}_{\left(V T_{1}\right.}[k], V T_{2}[k]\right), 1 \leq$ $k \leq N$.

Vector time is the most widely used strong clock to capture the causality among event. Its correctness is given by Theorem 2.1.1 [126]. Moreover, if the process where the event occurs is known, Theorem 2.1.1 can be rewritten as Corollary 2.1.1 [126].

Theorem 2.1.1. For two events $e_{l}$ and $e_{2}$, we have

1. $e_{1} \rightarrow e_{2}$ iff $V T\left(e_{1}\right)<V T\left(e_{2}\right)$.

2. $e_{1} \| e_{2}$ iff $\neg\left(V T\left(e_{1}\right)<V T\left(e_{2}\right)\right)$ and $\neg\left(V T\left(e_{2}\right)<V T\left(e_{1}\right)\right)$. 
Corollary 2.1.1. For two events $e_{1} \in E_{i}$ and $e_{2} \in E_{j}, e_{1} \neq e_{2}$, we have

1. $e_{1} \rightarrow e_{2}$ iff $V T\left(e_{1}\right)[i] \leq V T\left(e_{2}\right)[i]$.

2. $e_{1} \| e_{2}$ iff $V T\left(e_{1}\right)[i]>V T\left(e_{2}\right)[i]$ and $V T\left(e_{2}\right)[j]>V T\left(e_{1}\right)[j]$.

For a distributed system which consists of $N$ processes, the size of the vector time of each event is, therefore, $N$. Research efforts have been made to address its size from two aspects. From the aspect of reducing communication cost, it is desirable to reduce the piggybacked information which is sent along with messages. Approach proposed in [127] sends only different entries in vector time compared with the last transmission to the destination and restores vector time at the receiving process. It can largely save communication bandwidth in many cases, however, the transmission channels are required to be FIFO and the restoration of vector time takes $O\left(N^{2}\right)$ space at each process. It is also impossible to determine the causal order between send events only based on the piggybacked information they carry [101]. Further reduction can be found in [54] where the time diagram of distributed computation is regarded as a directed graph and events are treated as its vertices. Since each receive event has only one corresponding send event from another process, $O(1)$ piggybacked information is enough to fully determine the whole graph. This approach saves communication cost to an extreme. But restoration of the vector time of events, by either depth-first retracing [54] or breadth-first retracing [S], comes to another extreme. The overhead of restoration may be too large for on-line computation. Nevertheless, it might be suitable for off-line analysis of causality.

From the aspect of reducing the size of vector time itself, pessimistic result [27] shows that $N$ is the lower bound size to characterize the causality using vector. This is a general result. The size of vector time, however, still can be reduced if some other information, e.g., specific topology of communication, can be employed. By introduction of propagators, size $N$ can be decreased to an arbitrary size between 2 and $N$ [134]. 
plausible clock [142] fixes the size of vector time to a value (smaller than $N$ ) that is independent of the number of processes in the distributed system. However, plausible clock proves to be a weak clock.

\subsubsection{The Causal Order Delivery}

From the above discussion on logical clocks and the causal order, it is apparent that only a strong clock can be used to identify whether or not two events are causally ordered and the lower bound of the space complexity of strong clocks is $O(N)$. To perform the causal order delivery, however, more information are needed.

Definition 2.1.9 (The Causal Order Delivery). For any two messages $m_{1}$ and $m_{2}$ that are sent to the same destination $P_{i}$ if $S_{m_{1}} \rightarrow S_{m_{2}}, m_{1}$ must be delivered to $P_{i}$ before $m_{2}$, i.e., $R_{m_{1}} \rightarrow R_{m_{2}}$ must also be guaranteed at $P_{i}$.

In the above definition, $S_{m}$ and $R_{m}$ denote the send event and receive event of message $m$ respectively.

In the literature, there are many algorithms to implement the causal order delivery. They have different costs for their different assumptions of the underlying communication characteristics.

In broadcast environment, the approach introduced in [12] was firstly adopted in ISIS system [13], which piggybacks whole causal history of event with messages. Although some optimizations effectively reduce the size of causal history, in general, the approach has a very high cost. Later version of ISIS system adopts vector time and the size of piggybacked information was reduced to $O(N)$ [14].

In point-to-point environment, the approach using message-count-vectors [73] has the complexity of $O(N)$ for a single server process. To guarantee all messages to all processes to be delivered in the causal order, the complexity of piggybacked information is $O\left(N^{2}\right)$, 
which is the same as that of matrix time [118] and its variant [125].

By exploiting the topology of the underlying communication network, algorithms proposed in $[121,136,137]$ simplify the causal order delivery and has lower complexity. However, they are limited to certain range of applications. Other algorithms, e.g., the one reported in [104], require additional synchronization activities, thus result in a loss in concurrency.

To be general, algorithms applicable in multicast environment ${ }^{2.1}$ are required. Adaptive causal ordering algorithms $[78,112]$ introduce concepts of the causal barrier (CB), which captures the immediate causal-predecessors of the sending message; and the delivery matrix which stores the process's knowledge of the latest messages delivered to other processes. Compared to matrix time, adaptive causal ordering algorithms significantly reduce the piggybacked information (including the $\mathrm{CB}$ ). As a further optimization, a vector time based algorithm can be found in [19], which further optimized the CB and the delivery matrix at each process $\left(O\left(N^{2}\right)\right.$ ) is replaced by vector time $(O(N))$.

The notions of the causal order and the causal order delivery are fundamental to our research presented in Chapter 3, Chapter 5 and Chapter 6, in which they will be further discussed within different problem domains.

\subsection{Distributed Virtual Environments}

\subsubsection{Applications}

Over the past two decades, the following three fields have become prominent in the development of DVEs [129]:

\footnotetext{
${ }^{2.1}$ Broadcast and point-to-point environments can be regarded as the special case of multicast environments in which the number of destinations of each message is $N$ and 1 respectively.
} 
- Military applications: It has been well realized that virtual environments are of great benefit to military for the purpose of training and weapon testing. With the help of synthetic battle terrain, landscapes and military forces, participants are able to gain battle experiences without dangers to their lives and destructions to natural environment.

The United States Department of Defense developed the first protocol for military simulations, namely, SIMNET [102], in 1980s. By extending SIMNET, Distributed Interactive Simulation (DIS) was issued as IEEE standards [68, 69] and used in military training systems such as NPSNET [90] and PARADISE [67, 128]. The current military research concentrates on the High Level Architecture (HLA [70]), which puts more emphasis on interoperabilities among different types of simulations (both military and non-military applications).

- Collaborative virtual environments (CVEs): CVEs are the result of a convergence of research work in virtual reality (VR) and computer supported cooperative work (CSCW). Different from military purpose DVEs, which aim to build diverse large-scale system and support thousands of participants, CVEs support relatively small number of participants and pay more attention to their collaboration and resource sharing.

DIVE [65] is a fully distributed heterogeneous VR system where users navigate in 3D space and may see, meet and interact with other users and applications in the environment. MASSIVE $[61,62]$ is a VR conferencing system where participants communicate with each other by means of graphics, audio and text. REDUCE [133] and GRACE [ 135] are real-time cooperative editing systems which allow physically dispersed people to collaboratively edit text and graphical documents respectively.

- Multi-player computer games: Multi-player games date back to the adventure 
and multi-user dungeon games (MUD) in 1980's, and nowadays see their success in commercial entertainment markets. However, only until recently have the problems of multi-player games, particularly the ones in distributed environments, started drawing researchers' attention.

Artery [30, 31] aims to simplify the development of interactive network games by providing shared-memory programming interface that built on top of DIS protocol. MiMaze [41] is a distributed (serverless) 3D shoot game that employs unreliable communication system that is based on Real-Time Transport Protocol (RTP) over UDP/IP multicast. Distributed Entertainment Environment (DEE [110]) sets its goal to design and implement a DVE architecture that can support a graphical MUD.

\subsubsection{Distributed Systems Support}

\section{Distribution of Data}

There are three different ways that data are distributed to the sites in a DVE 191,1231 .

- unicast : Unicast is also known as point-to-point communication. The sender sends a message to exactly one recipient.

- multicast: Multicast provides one-to-many and many-to-many delivery services. The sender sends a message to its recipients, an arbitrarily-sized subset of the sites in the DVE, via a single transmission.

- broadcast: Broadcast provides one-to-all delivery service. The sender sends a message that may be received by all the sites in the DVE. 


\section{Communication Models}

The communication models of existing DVEs can be broadly classified to client/server model, peer-to-peer model and hybrzd model [91,123].

- client/server: Client/server model is the simplest model to build the majority of small-scale DVEs. Due to centralized management of information, consistencies are relatively easy to be maintained. Client/server model sees its success in cornmercial DVEs, e.g., commercial network game service providers, where hundreds of clients can be served simultaneously. However, due to the bottleneck at the server, client/server model is not scalable for large-scale DVEs.

- peer-to-peer: Instead of a central server providing services for clients on the network, peer-to-peer model considers all the sites to be equal. Any two sites can share information directly with each other and make their own control decisions without the need or delay of using an intermediate site. This model dramatically increases the difficulties in maintenance of consistency but makes the DVE well scalable for thousands of sites.

- hybrid: Hybrid model merges above described client/server and peer-to-peer models.

\section{Data Models}

A DVE can be viewed as being built upon a collection of objects, e.g., human participants, collaboratively manipulated documents, projectiles, vehicles, environmental elements etc. With the knowledge of the means by which the data are distributed and communication models through which the sites are interconnected, the ways to manage the objects' data in a DVE, also known as data models, can be categorized as shown in Figure 2.2 [95]. 


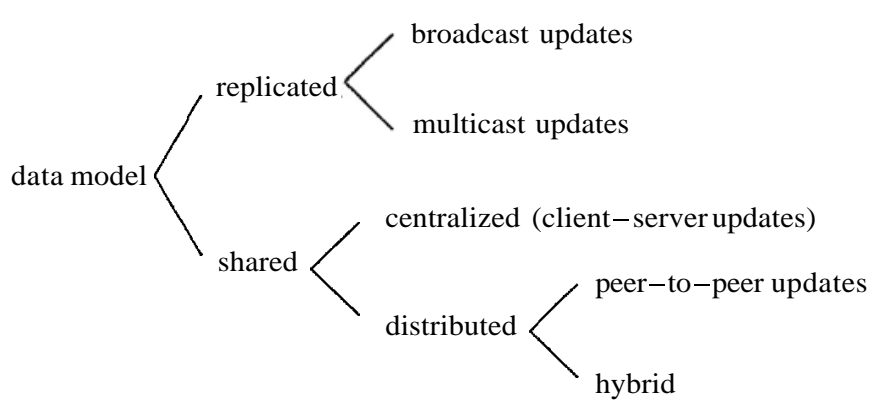

Figure 2.2: Data models of DVEs

The data models for DVEs may be either replicated or shared. In the replicated data model, all sites in the DVE initialize their worlds from a common database and hereafter maintain their own copies with incoming update information from others. Update information are usually broadcast or multicast to sites. In the shared data model, the whole collection of objects' data are stored in a centralized or distributed databases. Each site sends their updated information to or receives their updating information from the database(s). It can be seen that replicated data model and shared data model using distributed databases are potentially more scalable. However, they give rise to more challenges in maintaining consistencies.

A variant of the above data models is the distribution models categorized in [16]:

- active copying: A new object or any change to an existing object at a site are actively distributed among all other sites.

- copying on demand: A new object or any change to an existing object is only distributed to another site if that site expresses its interest in that object.

- migration combined with partial copying: In contrast to above two methods, objects are only distributed but not duplicated. An object may be transferred from one site to another to meet certain criteria such as achieving load balancing and decreasing accessing time for frequently accessed objects. 
As their names imply, copying means that objects are distributed among sites (corresponding to the replicated data model) while migration means that the objects is not duplicated (corresponding to the shared data model).

\subsubsection{Consistency Models}

There are many consistency models for existing distributed systems such as distributed shared memory, distributed file systems and world wide web ( $W W W$ ), which allow distributed users to access shared data that is either cached or replicated at multiple sites. Many of these models abstract the access to the data as read and write operations and require certain ordering of these operations to be ensured at different sites.

Sequential consistency [81] requires that all operations by all sites on the data appear to execute in some serial order where the order of operations of each individual site is preserved. Linearizability [66] requires a stronger serial order that must also respect the time induced order between operations of different sites. Causal consistency [2] resorts to a weaker consistency where all sites are required to see potentially causally related operations in the same order. Several models, namely, release consistency [43] and entry consistency [10] were also proposed to weaken the ordering constraints for operations in distributed shared memory systems, but all of them require additional synchronization operations.

The above mentioned models can also be useful in ensuring consistencies in DVEs, however, timely execution of DVEs is of the same importance. It is known that consistency and timeliness are orthogonal requirements in distributed systems. Timeliness is generally satisfied by sacrificing consistency requirement for less communication overheads and synchronization among sites. Stronger consistency, however, is generally achieved by reducing timeliness so as to provide more synchronization among sites. Any consistency model in DVEs is principally a tradeoff between these two requirements, thus, 
the consistency that the model can provide may have multiple levels.

Recall the data models shown in Figure 2.2, one way to categorize consistency models adopted in the existing DVEs, as summarized in Table 2.1 [95], is according to their abilities to maintain consistent views of the same objects from different sites in a DVE. Weak consistency models, as opposed to strict consistency models, cannot prevent worlds at different sites from becoming inconsistent over time.

\begin{tabular}{llllllll}
\hline & SIMNET & NPSNET & CAVE & DIVE & MASSIVE 2 & mWorld & Spline \\
\hline $\begin{array}{l}\text { data } \\
\text { model }\end{array}$ & repli. & repli. & $\begin{array}{l}\text { shared, } \\
\text { dis. }\end{array}$ & $\begin{array}{l}\text { shared, } \\
\text { dis. }\end{array}$ & repli. & $\begin{array}{l}\text { shared, } \\
\text { dis. }\end{array}$ & $\begin{array}{l}\text { shared, } \\
\text { dis. }\end{array}$ \\
\hline $\begin{array}{l}\text { data } \\
\text { repli. }\end{array}$ & full & full & full & partial & partial & full & full \\
\hline $\begin{array}{l}\text { consis. } \\
\text { model }\end{array}$ & weak & weak & weak & strict & weak & strict & weak \\
\hline
\end{tabular}

repli. $=$ replication, dis. $=$ distributed, consis. $=$ consistency

Table 2.1: Data models and consistency models in various DVEs

By exploring the relation between two elementary factors in DVEs, namely, the evolvement of objects' states and time advancement, consistency models in replicated DVES can be categorized from two separate domains, namely, the discrete domain and the continuous domain [99, 100], and each domain expects different consistency maintenance mechanisms.

\subsubsection{Consistency Maintenance of Replicated DVEs in the Dis- crete Domain}

The key observation in replicated DVEs in the discrete domain is that the objects in a DVE can be modelled as being changing their states discretely and only in response to user initiated operations.

Most applications of CVEs belong to this category. First of all, these applications 
require strong consistency to support collaborations. Furthermore, because timely execution of the operations is less critical, more overheads are acceptable in providing stronger consistency maintenance. Some general consistency maintenance mechanisms, e.g., locking, transaction, turn-based protocols, dependency-detection, reversible execution and master entities, can be applied.

Particularly in the area of real-time collaborative editing systems, Sun, etc. have indepth study in the consistency model and consistency maintenance in shared text editors and drawing tools $[135,136]$.

A collaborative editing system is said to be consistent if the following consistency properties are always maintained:

1. Causality-preservation: For any pair of operations $O_{a}$ and $O_{b}$, if $O_{a} \rightarrow O_{b}$, then $O_{a}$ is executed before $O_{b}$ at all sites.

2. Convergence: When the same set of operations have been executed at all sites, all copies of the shared document are identical.

3. Intention-preservation: For any operation $O$, the effects of executing $O$ at all sites are the same as the intention of $O$, and the effect of executing $\boldsymbol{O}$ does not change the effects of independent operations.

Note that these three consistency properties are independent from each other in the sense that satisfying any one or two of them does not always result in the others. With the help of Figure 2.3, maintenance mechanisms of these properties are described as follows.

1. Achieving causality-preservation: This is actually the problem of the causal order delivery discussed in Section 2.1.3. The assumption of broadcast in editing systems simplifies the algorithm so that vector time is sufficient to deliver causally 


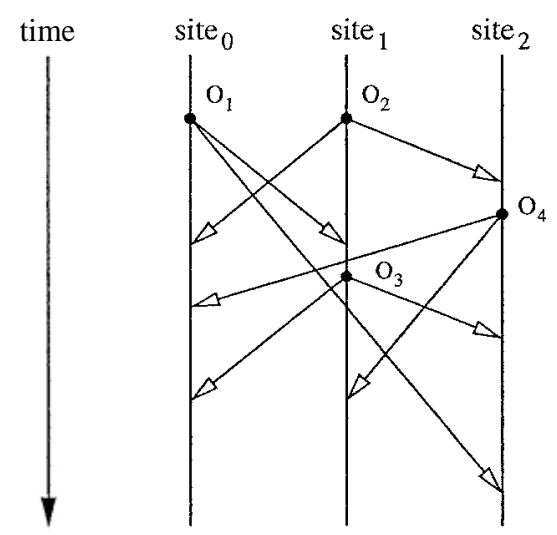

Figure 2.3: A scenario of a real-time editing session

related operations at all sites. As shown in Figure 2.3, $\mathrm{O}_{1} \rightarrow \mathrm{O}_{3}, \mathrm{O}_{2} \rightarrow \mathrm{O}_{3}$ and $\mathrm{O}_{2} \rightarrow \mathrm{O}_{4}$, the delivery order of these three pairs of operations at all sites is ensured that $O_{1}$ before $O_{3}, O_{2}$ before $O_{3}$ and $O_{2}$ before $O_{4}$ respectively.

2. Achieving convergence: Causality-preservation only orders causally related operations. For concurrent ones, e.g., $\mathrm{O}_{1} \| \mathrm{O}_{2}$ and $\mathrm{O}_{3} \| \mathrm{O}_{4}$ in Figure 2.3, they can be artificially ordered by their originating sites' identifiers. This allows total ordering of all operations among all the sites. With the assumption that all operations in editing systems are reversible, convergence is achieved in the sense that the sequence of totally ordered operations, e.g., $\mathrm{O}_{1} \mathrm{O}_{2} \mathrm{O}_{3} \mathrm{O}_{4}$, can be ensured to be identically preserved at all sites by an undo/do/redo scheme.

3. Achieving intention-preservation: This is known much harder than achieving causality and convergence-preservation, because intention-preservation cannot be achieved by simply reordering of operations. As shown in Figure 2.3, for example, the execution of $\mathrm{O}_{4}$ at site $_{0}$ may be different from the intention of generating $\mathrm{O}_{4}$ at site $_{2}$ due to the existence of a concurrent operation, $O_{1}$, which could have brought the document's state at site $e_{0}$ into a different one. By using application specific knowledge in editing systems, exclusion transformation and inclusion transforma- 
tion can be performed over concurrent operations to ensure these operations are generated from the same document state [136].

\subsubsection{Consistency Maintenance of Replicated DVEs in the Con- tinuous Domain}

The key observation in replicated DVEs in the continuous domain, however, is that the objects in a DVE change their states not only in response to user initiated operations but also because of the passing of time. This implies that there is generally no way to identically replicate a continuous object, e.g., a projectile or a moving vehicle, at multiple sites due to communication latency (once a continuous object is replicated at another site, the original one may have evolved to a new state), which gives rise to the major consistency problem in the continuous domain applications and is refer to as time-space consistency.

In addition to the above mentioned time factor, which can be thought of as a physical law, there are also other two major reasons that make consistency maintenance not easy to be tackled. The first reason, most applications in the continuous domain are actually military purpose DVEs and multi-player games. In order to provide participants interactions that are comparable to the real ones, all these applications consider timeliness to be a paramount requirement. Therefore, synchronization overheads involved in consistency maintenance have to be limited or avoided. The second reason, applications in the continuous domain demand much more bandwidth than those in the discrete domain. For example, an object continuously updates its state at one site and broadcast the update packets, fixed at $40 \mathrm{~Kb}$, to other sites. If the broadcast is carried out at frame rate 25 fps, the update of this single object requires $1000 \mathrm{Kbps}$ of network bandwidth. In order to support thousands of objects in large scale DVEs, e.g., NPSNET and STOW, high frequency updates are to be avoided and consistency maintenance mechanisms consuming 
less bandwidth are required.

In summary, consistency maintenance in the continuous domain is generally more difficult than that in the discrete domain. With bandwidth limitation and the promise of timeliness, consistency maintenance mechanisms have to be compromised and adjusted for real application's requirements. Several existing approaches are discussed below. In Chapter 3, our proposed consistency maintenance scheme based on event ordering will be elaborated.

\section{Dead Reckoning}

The objective of using dead reckoning $[18,41,44,68]$ is to minimize network traffic while maintaining a reasonably consistent view of an original object at remote sites. Ever since SIMNET adopted dead reckoning technique $e^{2.2}$, it has proved to be successful in maintaining consistency among a huge number of objects in large scale DVEs.

The most common prediction technique is to use derivative polynomials based on the object's movement equation. For First order derivative polynomials, only the velocity and position of the object are transmitted to remote sites. For second-order derivative polynomials, transmission of the acceleration of the object is also required. A variant approach is the position history-based dead reckoning (PHBDR [128]), which only transmits the object's absolute positions, of which the velocity and acceleration are approximated by recently received updates. There are basically two approaches to determine the rate to transmit updates. Heart-beating [68] simply transmits updates at fixed rate, which normally requires higher network bandwidth for higher fidelity. The other way to determine when to send update is to compare the object's real position with that calculated using the dead reckoning model. The update is sent if the difference is greater than a

\footnotetext{
${ }^{2.2}$ In SIMNET, it is reported that 100,000 objects would require 375 Mbps of network bandwidth to each site [92].
} 
predefined threshold value.

\section{Time-space Inconsistency Metric}

A quantitative analysis of time-space inconsistency caused by communication latency in dead reckoning algorithms can be found in $[152,153]$. Time-space inconsistency $(\Omega)$ between an object's local position and its estimation by a remote site in average dead reckoning update interval $\left(T_{D R}\right)$ is computed as a function of wallclock drift $(\gamma)$, dead reckoning threshold (6), average communication latency $(T d)$, the object's average velocity $(\bar{v})$ and maximum acceleration $\left(|a|_{\max }\right)$ :

$$
\Omega=\bar{v} \cdot \gamma \cdot T_{D R}+\frac{1}{2} \cdot \delta \cdot\left(T_{D R}+T_{d}\right)+|a|_{\max } \cdot T_{D R} \cdot \frac{1}{2} \cdot T_{d}^{2}
$$

\section{Short-term Inconsistency, Response Time and the Local Lag}

Short-term inconsistency in the continuous domain refers to the situation where the state of at least two sites is different from each other because an operation issued at the source site arrives at the other site after the time at which the operation is supposed to be executed due to communication latency. The site receiving a "past" operation is required to repair its state, which usually results in short-term visual artifacts.

The response time of an operation is defined as the wallclock time difference between the operation is issued and executed at the source site. It is clear that low response time (high responsiveness) is ideal for the participant who issues the operation at the source site. However, low response time also more likely causes short-term inconsistencies. As shown in Figure 2.4 (a), assuming that all sites have perfectly synchronized clocks and considering the extreme case, namely, zero response time, because communication latency is nonnegligible, all three sites will experience short-term inconsistencies at time executing any operation $\left(O_{1}\right.$ and $\left.O_{2}\right)$. 


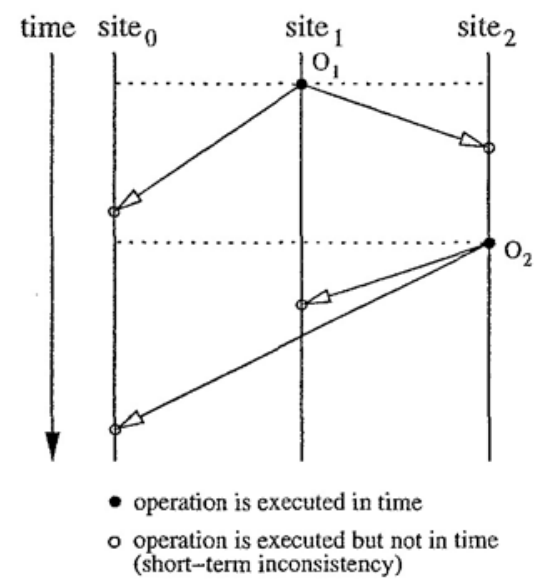

(a)

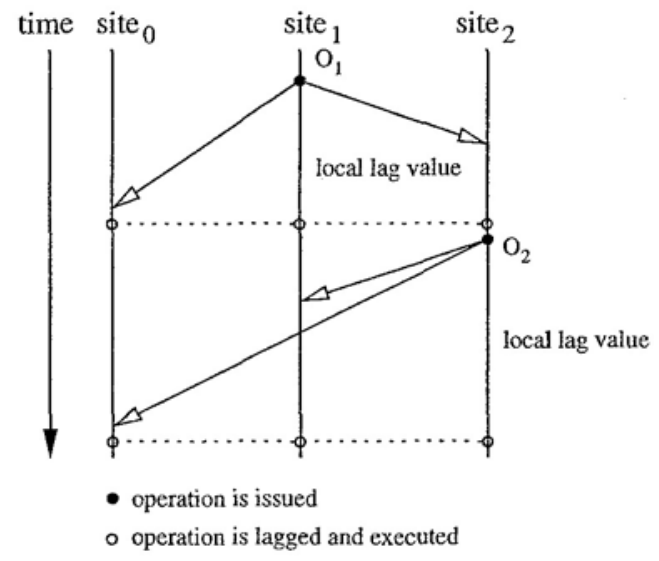

(b)

Figure 2.4: Short-term inconsistency and the Local Lag

To alleviate short-term inconsistencies that would be experienced by participants, the local lug $[34,99,100]$ is based on the following idea: instead of immediately executing an operation issued by the source site, the execution of the operation is intentionally delayed for a certain period of time at the source site, i.e., by increasing response time, to compensate communication latency that the operation is suffered when executing at remote sites (see Figure 2.4 (b)).Bigger value for the local lag is more helpful in removing short-term inconsistencies. However, there is an upper bound to be realistic. It is reported that human reaction time is about $200 \mathrm{~ms}$ [145], which means response time exceeding this threshold would cause significant lag perceivable by participants. Therefore, to determine a value for the local lag, three steps are normally taken: (1) Determine a minimum value for the local lag to prevent majority of short-term inconsistencies, then (2) Determine the highest acceptable response time, and finally (3) Choose a value for the local lag as a compromise. 


\subsection{Analytic Parallel and Distributed Simulation}

\subsubsection{Simulation Principles}

The notions of State, Time and Event

A simulation ${ }^{2.3}$ is a computer program that is used to represent or emulate the behavior of a physical system over time. The physical system may be an existing system, or it may be a conceptual one.

State, time and event are fundamental notions when discussing a simulation. The notion of state in a simulation can be interpreted as two requirements: (1) The simulation needs to provide a computerized representation of the simulated physical system; and (2) The simulation also needs to provide some means of changing this representation in order to mimic the evolvement of the system. Empirically, the representation is through a collection of state variables, which are actually instantiated program variables using particular programming language. The changes to the representation is reflected by updating new values into these state variables. The notion of time has several definitions and should be distinguished in different context. Physical time refers to the time in physical systems; Wallclock time refers to the time during the execution of a simulation; Simulation time, or virtual time, refers to the abstract representation of physical time in simulations, which is formally defined in Definition 2.3.1 [59].

Definition 2.3.1 (Simulation Time). Simulation time is defined as a totally ordered set of values where each value represents an instant of time in the physical system being modelled. Further, for any two values of simulation time $S T_{1}$ and $S T_{2}$ representing physical time $P T_{1}$ and $P T_{2}$ respectively, $\left(S T_{1}-S T_{2}\right)=\lambda \times\left(P T_{1}-P T_{2}\right)$ for some constant $\lambda$. If $S T_{1}<S T_{2}$, then $S T_{1}$ is said to occur before $S T_{2}$, and if $S T_{1}>S T_{2}$, then

\footnotetext{
${ }^{2.3}$ For the simplicity and without explicit statement, the term "simulation" refers to "analytic PADS" , or more specific, "parallel and distributed discrete event simulation" hereafter without any ambiguity in the context.
} 
$S T_{1}$ is said to occur after $S T_{2}$.

Definition 2.3.2 (Simulation Event). A simulation event is defined as a time-stamped action (an action with simulation time) processed by an LP.

Note that the concept of simulation event is similar with the concept of event defined in Definition 2.1.2. The difference comes from different conventions used in distributed system community and simulation community. For example, a simulation having time-

space diagram identically drawn as Figure 2.1 interprets $e_{22}$ and $e_{32}$ as $e_{22}$ schedules $e_{32}$ through message $m$ rather than $e_{22}$ and $e_{32}$ are send event and receive event of message $m$. Nevertheless, the concept of enumeration in Definition 2.1.2 and the theories of the causal order etc. that follow can also be applied to simulation event. For this reason, the term event is used throughout the thesis and its meaning is distinguished from its context.

\section{State Changes and Time Flow Mechanisms}

As discussed above, the evolvement of a physical system is modelled as a collection of state variables that change in simulation time. According to the way the state changes, a simulation can be categorized by continuous model and discrete model. In a continuous simulation, the state of the system is typically described as a set of differential equations and viewed as changing continuously over simulation time. While in a discrete simulation, the state of the system only changes at discrete points over simulation time.

A discrete simulation can be further categorized into time-stepped model and eventdriven model according to the way the time advances. In a time-stepped simulation, simulation time is subdivided into a sequence of time steps of constant size $\Delta$, and the simulation only advances from one time step to the next. While in an event-driven simulation, the simulation advances by continuously processing time-stamped events and 
scheduling new events in the future. It is well known that these two models can be transformed to each other. However, discrete event-driven simulation is generally more natural and efficient in modelling physical systems. Therefore, it receives broad study.

\section{Sequential, Parallel and Distributed Simulation}

A sequential simulation typically uses three data structures: (1) a global clock variable indicating current simulation time, (2) a time ordered event list holding time-stamped events scheduled to occur in the future, and (3) a collection of state variables describing the current state of the simulated physical system. These data structures are generally maintained using a two-layer simulation architecture. At the lower layer, the simulation executive provides application independent support for event list management and simulation time advancement. At the higher layer, the application provides application specific support such as state variables and application code that model the behavior of the physical system. For the seek of correctness, it is required that not only the simulation executive always processes events in ascending time-stamp order, but also the application always schedules events in simulated future, i.e., the time-stamp of a newly scheduled event must be no smaller than current simulation time.

The research of sequential simulation dates back to the 1950s and has been a mature field including numerous areas, e.g., model design and development, programming languages and experimental design [52,82]. However, given the high computational demands of large simulations and availability of high performance multiprocessors, multicomputers and geographically dispersed computers, parallel and distributed Simulation, as defined in Definition 2.3.3, proves to be a promising approach to promote simulation performance and address scalability problems.

Definition 2.3.3 (Parallel and distributed simulation). The physical system is viewed as being modelled by a number of logicalprocesses (LPs), denoted by $L P 1, L P_{2}, \ldots$, 
$L P_{N^{*}}$. These LPs may or may not share memory, however, interactions among LPs are solely carried out by event scheduling using message passing through transmission channels connecting corresponding LPs. Let $m$ and m.e denote the message and its scheduled event respectively. The message is time-stamped with the event's simulation time, i.e., $S T(m)=S T(m . e)$. Each $L P_{i}$ maintains a local virtual time (LVT), denoted by $\operatorname{LVT}\left(L P_{i}\right)$. Once an event $\mathrm{e}$ is processed at $L P_{i}$, the LVT of the LP advances and is updated with $L V T\left(L P_{i}\right)=S T(e)$.

Definition 2.3.4 (Local Causality Constraint). A parallel and distributed simulation as defined in Definition 2.3.3 obeys the local causality constraint if and only if each LP processes events in nondecreasing time-stamp order (TSO).

Note that adherence to the local causality constraint (LCC), as defined in Definition 2.3.4, is crucial to the correctness of parallel and distributed simulation in the sense that it is sufficient for a parallel and distributed simulation to produce exactly the same result as running the simulation in a sequential way. The LCC states that for any two events e and $e^{\prime}$ scheduled at $L P_{i}$, if $S T(e)<S T\left(e^{\prime}\right)$, then e must be processed before $e$ ', because their simulation time implies a causality constraint of $\mathrm{e}^{\prime}$ over $e$, i.e., the processing of e' may depend on the state resulted from the processing of $e$.

To obey the LCC at each LP, synchronization protocols have been broadly categorized into two categories, namely, conservative synchronization and optimistic synchronization. Conservative synchronization, as its name implies, works in a way that it never violates the LCC. For any $L P_{i}$, it does not process an event until it ensures that it is safe to do so, i.e., for any future event $e$, it always holds that $S T(e) \geq L V T\left(L P_{i}\right)$ at time processing that event. Optimistic synchronization, however, allows temporary violations of the LCC to occur to exploit more parallelism among LPs. All these temporary violations must be corrected at runtime using a rollback mechanism. These two categories of synchronization 
protocols have their own assumptions, advantages and limitations, which are discussed in the following sections.

\subsubsection{Conservative Synchronization}

Original works on conservative synchronization can be found in [17, 24], and are often referred to as the Chandy-Misra-Bryant (CMB) protocol. In CMB, it is assumed that (1) The transmission channels are static, reliable and FIFO, and (2) The time-stamps of message sent over a transmission channel is nondecreasing. This implies that, from the point view of each LP, messages coming from any channel will always have nondecreasing time-stamps. To ensure the LCC, each LP needs only extract the message that has the smallest time-stamp among all the incoming channels to process ${ }^{2.4}$. If one of the incoming channels is empty, the LP has to block and wait until there is at least one message coming from that channel.

\section{Deadlock Avoidance}

Unfortunately, this "blocking-until-safe-to-process" strategy has hazard of deadlock. For example, if there exists a waiting cycle where each LP is blocked and waiting for a message coming from its neighbor LP, none of LPs could advance. Intuitively, deadlock may happen more frequently when there are less unprocessed messages compared to the number of transmission channels or the unprocessed message distributed unevenly in the channels.

CMB employs null-message [103], a time-stamped message that schedules no event and is solely used for synchronization purpose, to prevent deadlock from happening. The idea is that after processing any message, including null-message itself, at an LP, a nullmessage is always generated and sent along with all its outgoing channels. This ensures

\footnotetext{
${ }^{2.4}$ Incoming and unprocessed messages are assumed to be queued in channels.
} 
that, at any time a message is processed, at least one LP can have all non-empty incoming channels and, therefore, can continue the simulation. As long as there is no closed cycle of channels along which a message traversing does not increase its time-stamp, the nullmessage based protocol can be guaranteed to avoid deadlock [47].

A sufficient condition to prevent the above mentioned "nonincreasing-time-stampthrough-a-closed-cycle" situation is always to schedule messages, including null-messages, in simulated future, i.e., for any message $m$ sent at time $L V T\left(L P_{i}\right)$ by $L P_{i}$, it holds that $S T(m)>L V T\left(L P_{i}\right)$. The problem of how far in the future a message can be scheduled is generalized as the lookahead as defined in Definition 2.3.5. With knowledge of lookahead, each LP actively or passively sends out a null-message, carrying its current simulation time plus its lookahead, to help the simulation advance without being deadlocked.

Definition 2.3 .5 (Lookahead). If $L P_{i}$ at simulation time $L V T\left(L P_{i}\right)$ can only schedule new messages with timestamps of at least $L V T\left(L P_{i}\right)+L_{i j}$ through the channel to $L P_{j}$, then $L_{i j}$ is referred to as the lookahead for $L P_{i}$ to $L P_{j}$.

The performance of simulation may be severely degraded if a large number of nullmessages are overwhelming in the simulation. Optimization of null-message approach aims to reduce the frequency and amount of null-messages. In the demand-driven protocol [103], an LP blocked by an empty incoming channel actively requests the next message (null-message or otherwise) from the LP at the other side of the channel. This effectively reduces the amount of null-message traffic. The carrier null-message protocol [20] attaches more information, essentially route data, to null-messages. It reduces the null-message traffic by improving ability of lookahead using the route data. Another optimization, as proposed in [114], attempts to reduce the propagation of null-messages by eliminating stale ones. 


\section{Deadlock Detection and Recovery}

Rather than to avoid deadlocks using null-messages, an alternative approach [25] is to allow occurrence of deadlocks but detect them and recover from them. Each detection and recovery cycle involves the following phases: (1)Parallel execution. LPs are running in parallel until a deadlock is found by using any general distributed termination detection algorithm [96] or distributed deadlock detection algorithm [26]; (2) Deadlock resolution. Once a deadlock is found, the smallest time-stamp of the unprocessed message in the simulation is determined and made known to all the LPs. Therefore, LPs waiting on message(s) with this time-stamp can recover from the deadlock.

Compared to the null-message, the major advantage of deadlock detection and recovery approach is that heavy traffic of null-messages is avoided while the lookahead is not required to be non-zero.

\section{Barrier Synchronization}

Instead of recovering from a deadlock only when it occurs, barrier synchronization periodically controls the advancement of all the LPs and determines the set of safe events to be processed next, thus avoids most of deadlocks and increases parallelism in processing events.

A barrier, which can be implemented using global variables in shared-memory multiprocessor or protocol messages in distributed-memory computers [105, 109, 144] respectively, is a construct that defines a condition by which all the LPs should have stopped temporarily. Reaching a barrier means that there is no event being processed, no new event being scheduled and, by using a message counter technique, no transient message (the message that has been sent but has not yet been received) in the simulation, thus the set of safe events to be processed next can be determined. 
Let $x_{0} x_{1} \ldots x_{k}$ denote a $p a t h$ existing from $L P_{x_{0}}$ to $L P_{x_{k}}$ and $d_{x_{0} \ldots x_{k}}$ denote $\sum_{p=0}^{k-1} L_{x_{p} x_{p+1}}$. The distance [5] from $L P_{x_{0}}$ to $L P_{x_{k}}$, denoted as $D_{x_{0} x_{k}}$, is defined as $\underset{\text { all path from } x_{0} \text { to } x_{k}}{\min } d_{\text {path }}$. Let $\operatorname{LBTS}\left(L P_{i}\right)$ be the lower bound on the time-stamp of any message that $L P_{i}$ will receive and $S T_{\text {next }}\left(L P_{i}\right)$ be the smallest time-stamp of unprocessed messages in $L P_{i}^{2.5}$, $\operatorname{LBTS}\left(L P_{i}\right)$ can be computed by

$$
\operatorname{LBTS}\left(L P_{i}\right)=\min _{\text {all } j \neq i}\left(S T_{n e x t}\left(L P_{j}\right)+D_{j i}\right)
$$

Thus all unprocessed messages in $L P_{i}$ with time-stamp less than $\operatorname{LBTS}\left(\operatorname{LP}{ }_{i}\right)$ are safe.

The overhead of the LBTS computation can be reduced by using the bounded lug approach [89]. Given $\operatorname{milk}_{k} L V T\left(L P_{\mathrm{k}}\right)$, representing the smallest time-stamp after a barrier synchronization, and a time window $T_{\text {win }}$, only messages in the near future, i.e., $\left\{m \mid S T(m) \leq \min _{\text {all } k} L V T\left(L P_{k}\right)+T_{\text {win }}\right\}$, are considered in LBTS computation. Since for any $D_{j i}, D_{j i}>T_{w i n}, L P_{j}$ will not schedule any event in the near future of $L P_{i}$, the computation of $\operatorname{LBTS}\left(L P_{i}\right)$ involves less number of LPs.

$$
\operatorname{LBTS}\left(L P_{i}\right)=\min _{\substack{\text { all } j \neq i \\ D_{j i} \leq T_{w i n}}}\left(S T_{n e x t}\left(L P_{j}\right)+D_{j i}\right)
$$

It is observed that performance of conservative simulation, no matter what protocol is used, is heavily depending on the lookahead property exhibited by each LP [56]. The bigger the lookahead, the larger amount of safe messages can be processed in parallel. Even so, the simulation may still advance overly conservatively because of the blacking and safe-to-process rationale to maintain the LCC . On the other hand, the reliance on the lookahead, which is often application dependant and varies with different modelling techniques, ties performance gain with particular simulation model. Any further mod-

\footnotetext{
${ }^{2.5}$ If there is no unprocessed message in $L P_{i}$, then $S T_{n e x t}\left(L P_{i}\right)=\infty$.
} 
ification to the model that results in the change of lookahead may consequently have significant impact on the performance.

\subsubsection{Optimistic Synchronization}

\section{Time Warp Simulation}

Time Warp simulation [71] was pioneering work in optimistic synchronization and remains the most well-known optimistic synchronization protocol nowadays. The basic idea is that, rather than strict adherence to the LCC, messages can be processed in a speculative (as-fast-as-possible) way, avoiding safe event determination and maximizing parallelism.

A rollback mechanism is used for recovering from LCC violations. If a message $m$, from another $L P$, arrives in the past of $L P_{i}$, i.e., $S T(m)<L V T\left(L P_{i}\right)$, the message is named struggler message. The computations speculated during the period of $\left(S T(m) L V T\left(L P_{i}\right)\right]$ at $L P_{i}$ are identified as erroneous ones and need to be rolled back (primary rollback). Furthermore, because it is also likely that $L P_{i}$ has scheduled messages to other LPs during this period, these messages have to be unsent as well to finish the recovery.

The most basic way to cancel (unsend) a positive message is by sending its antimessage, which can be an exact copy of the former except for a flag indicating the difference. Upon receipt of both the positive message and anti-message, the process of annihilation takes place, which removes both messages from the receiving LP and rolls back (secondary rollback) the LP if the positive message has been processed. Other ways of cancelling messages will be thoroughly discussed in Chapter 5 . 


\section{State Checkpointing}

A state of an LP can be thought as a snapshot of the values of the LP's state variables at a certain wallclock time. The rollback mechanism requires the chronical evolvement of an LP's states, received messages and scheduled messages to be queued so that rolling back to any old state and restarting computation from there become possible.

There are two common techniques to save (checkpoint) states, namely, copy state saving and incremental state saving. In copy state saving approach, the states before and after processing an event are wholly copied and checkpointed. In incremental state saving approach, however, only modified state variable(s), as a part of the state, are queued. Incremental state saving typically reduces storage cost, but requires going through rolled back states in a decreasing time-stamp order when recovering an old state. Copy state saving can be done in a less frequent way, namely, infrequent state saving [55, 87], in which the state to be rolled back to does not necessarily exist. The LP probably needs a coast-forward phase, i.e., rolling back to an earlier saved state and reprocessing events afterwards, to reconstruct the state at the rollback point. Less frequent state saving reduces storage cost but increases rollback cost accordingly (coast-forward phases may take longer time). Let interleaving factor $\pi$ be the number of events processed divided by the number of rollbacks, the optimal interleaving factor of infrequent state saving $\pi^{*}$ is bounded by $\sqrt{(\alpha-1) \beta}<\pi^{*}<\sqrt{(2 \alpha+1) \beta}$ [87],where Q! is the average number of rollbacks with no interleaved state saving being applied and,$\beta$ is the ratio of the state saving cost and expected event processing time.

\section{Fossil Collection and GVT Approximation}

As a simulation is advancing, states, incoming messages and sc,,eduled messages queued at each LP may become obsolete, most Time Warp systems carry out the process of fossil collection to reclaim their memory periodically or when the simulation runs out 
of memory. The concept of global virtual time (GVT), as defined in Definition 2.3.6, serves the basis to determine obsolete states and messages in the simulation. Because a $G V T(t)$ represents the minimal simulation time in a global snapshot of the simulation, and because an LP always schedules messages in its future, it is safe to say that no LP will roll back prior to $G V T(t)$. Thus, any state and message having time-stamp smaller than $G V T(t)$ are said being committed and their allocated memory can be reclaimed.

Definition 2.3.6 (GVT). $G V T(t)$ at wall-clock time $t$ is defined as the minimum timestamp among all unprocessed and partially processed messages and anti-messages in execution of a Time Warp simulation at that $t$.

Due to the distributed nature of the simulation, GVT is normally computed approximately. Samadi's algorithm [124] performs centralized GVT approximation, assuming messages are acknowledged. Some other algorithms [86, 141] eliminate message acknowledgment but require reliable message delivery. pGVT algorithm [42] does not assume FIFO channels or reliable message delivery but, still, relies on message acknowledgment. LPs report their local save of GVT information to a central GVT manager initiatively based on their statistics of their historical advancement. The key improvement of pGVT is that LPs' reports of GVT information are adjusted according to their contributions to GVT's advancement. Mattern's algorithm [98]does not rely on central GVT server, FIFO channels or message acknowledgement. A distributed GVT approximation is carried out based on the concepts of global termination [23] and consistent cut.

\section{Variants of Time Warp Simulation}

The basic Time Warp simulation presented above provides unlimited optimism to LPs, thus exploits maximal parallelism in processing events. However, in situations that LPs advance unevenly, e.g., due to different computation power or work load, unlimited optimism may give rise to many performance pitfalls, e.g., cascading rollbacks, rollback echoes 
and unwanted multiple rollbacks ${ }^{2.6}$, which will severely degrade the performance or, even worse, destroy the simulation.

Several variants of the basic Time Warp simulation address these potential performance pitfalls by introducing certain conservativeness into optimistic advancement of LPs. Moving Time Window (MTW) [130]prevents an LP from lagging behind other LPs too far away by defining a window size $\Delta$. LPs perform optimistic computation within the window $[G V T, G V T+\Delta]$ and the window will be moved whenever GVT is recalculated. Bounded Time Warp (BTW) [143] divides the simulation duration time into a number of equal intervals. All events inside the current interval are simulated before moving to the next interval. Local Rollback [40] eliminates secondary rollbacks by not allowing messages to be sent until they are ensured not to be rolled back, i.e., their time-stamps are greater than current GVT. Therefore, anti-messages can be avoided in the local rollback approach and LPs only recover when receiving straggler messages. Breathing Time Buckets (BTB) [131]can be viewed as an extension of the sequential event list mechanism that allows optimistic parallel processing. B TB determines the event horizon, i.e., the smallest time-stamp of any new events that will be generated after GVT, and only allows in-between events to be processed without the possibility of rollback. Probabilistic Rollback [48] intentionally rollbacks an LP to GVT with a user-defined probability $p$ to prevent over optimism. Throttled Time Warp [139] aims to maximize performance gain through dynamic control of Time Warp optimism. Time Warp and conservative synchronization are hierarchically combined in [117] to support large scale simulations, where Time Warp is used in intra-cluster synchronization to exploit parallelism and maintain flexibility and Conservative Time Windows [6] is used in inter-cluster synchronization to prevent cascading rollbacks.

For the same purpose, we proposed a rollback optimal cancellation scheme and a

\footnotetext{
${ }^{2.6}$ These pitfalls will be further discussed in Chapter 5.
} 
causal order based Time Warp scheme to reduce rollbacks in Time Warp simulations. These two approaches are presented in Chapter 5 and Chapter 6 respectively.

\section{Performance Evaluation for Time Warp Simulation}

The principal advantage of Time Warp over conservative synchronization is that Time Warp offers the potential for greater exploitation of parallelism. Time Warp has proved successful in speeding up simulation of communication networks [21, 115], queuing networks [57], and digital logic circuits [15],among many others.

From the perspective of theoretical analysis, earlier performance evaluation was confined to two-processor Time Warp simulation [45,46]. Later work was carried out on arbitrary number of processors [64] and under limited memory constraints [36]. It is shown in [85] that, assuming state saving and rollbacks have zero-cost (which is not realistic) and incorrect computation never rolls back correct computation, the execution time of Time Warp using aggressive cancellation is identical to the critical path through the simulation and lazy cancellation can improve performance further. It is also shown in [88] that there exists simulation models for which Time Warp would outperform the CMB null-message approach by up to a factor of $\mathrm{N}$ using $N$ processors. The reverse, however, is not true. The CMB approach can only outperform Time Warp by a constant factor assuming constant rollback costs. In [138], an analytic model is established for analyzing the performance of Throttled Time Warp. It suggests that a certain controlled degree of LCC violations is desirable in achieving optimal performance.

From the perspective of empirical performance study, it has been realized that actual performance gain of a simulation is closely related to the modelling technique that maximally extracts parallelism for a parallel execution. Several benchmarks have been designed to model various applications for studying protocols and conducting performance evaluations for Time Warp simulation. 
- PHOLD: The parallel hold (PHOLD) [58] is based on the hold model that is widely used to evaluate the performance of sequential event list algorithms. In this model, the number of messages, namely, message population, are initially configured among $N$ LPs. It is assumed that processing of each message only results in scheduling another single message. Therefore, there are constant number of event scheduling threads cycling through out the simulation. At time processing any message, the time-stamp increment is governed by a user-defined stochastic distribution. The newly scheduled message is sent to any of the LPs, including the sender LP itself, with equal probability. Symmetric workload can be simulated by specifying a unique computation grain (coarse grain or fine grain) at each LP, while asymmetric workload can be achieved by varying computation grain at different LPs.

- Super ping: The Super ping model $[9,150]$ consists of $N$ LPs connected in a ring, where each LP has two incoming channels and two outgoing channels connected from/to its neighboring LPs respectively. The model is initiated with a fixed number of messages and their computation grain, then continues with messages circulating in the model.

- Torus network: An $N \times N$ torus network is a two-dimensional $N$-ary hypercube where each node has exact four neighbors. With one-to-one mapping of LPs to CPUs, $N^{2}$ LPs are required in the simulation. After a message is processed at a node (LP), the newly scheduled message has equal probability to be sent along any one of four outgoing channels [140].

- PCS network: In a personal communication service (PCS) [21, 22] simulation, the network is represented by cells and each cell is modelled as an LP. There are basically two types of models, namely, call-initiated and portable-initiated, to characterize the traffic in the network. In the call-initiated model, only the life cycle of each phone 
call is simulated whereas in the portable-initiated model more states of a portable are concerned. Particularly, a busy-lines status prevents a portable from receiving any new incoming call. In both models, initialization of new calls/portables and hand-off of calls/portables are modelled as time-stamped messages with certain distribution. The lack of busy-lines monitoring makes the call-initiated model less accurate. However, for a large number of portables per cell, in which the busy-line effect is small, the call-initiated model is more appropriate for its higher efficiency.

- Manufacturing model: According to this model [84], a manufacturing process is viewed as a sequence of production, assembling and testing stages, in which all the stations (processing station, control station, assembling station and testing station) and all the functional connectors (forker, merger and collector) are modelled as separate LPs. Different components of a product go through all the stages by means of messages scheduling and are finally integrated together. The time-stamp increment is zero at connectors and configurable for stations. Possible backward links exist in the production stage. In case a component is detected defective (with certain probability), it is either returned by a control station to the immediate preceding processing station or returned from the end of the stage to the start for a thorough rework.

\subsection{Summary}

In this chapter, our researc backgrounc is introduced in three different areas and a survey of concepts, techniques and experiment models is carried out accordingly. The theories presented in Section 2.1 serve as the basis for our theoretic study and are intensively used in Chapter 3, Chapter 5 and Chapter 6. Survey of consistency issue in Section 2.2 suggests that maintenance of consistency in DVE is generally challenging and there exist 
various approaches to meet different requirements. In Chapter 3, the requirement for direct follow order will be identified and explored. Survey of the Time Warp simulation in Section 2.3 not only helps us to abstract Time Warp mechanism and develop the extensible framework for Time Warp simulation kernel (elaborated in Chapter 4) for ease of executive/application development, but also motivates us to improve the performance of Time Warp simulations by addressing rollback issue. Further study on rollbacks in Time Warp and our proposed two approaches can be found in Chapter 5 and Chapter 6. 


\section{Chapter 3}

\section{Direct-Follow Order Delivery}

\subsection{Introduction and Motivations}

Applications running on a DVE normally involve a set of communicating sites. According to Definition 2.1.1, these sites are modelled as geographically distributed processes, where messages are used to exchange their states and application related information. However, due to the notable message communication latency (e.g., delay in the Internet environment can be as large as hundreds of milliseconds), messages may arrive at a process out of order and thus cause inconsistencies in the distributed applications.

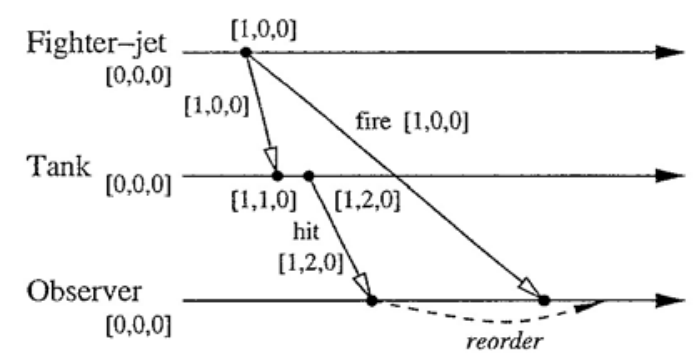

Figure 3.1: Inconsistency caused by violation of the causality

The cause of inconsistency can be seen as the result of processing arrived messages in a wrong order. For example, assume there are three participants in a distributed battlefield application: a fighter-jet, a tank and an observer (see Figure 3.1). These 
three participants are at geographically different locations and communicate by passing messages. Also assume that the fighter-jet fires at the tank at some instant in wall-clock time. The "fire" message is broadcast to both the tank and the observer. To respond to the "fire" message, a "hit" message is then generated by the tank and sent to the observer. Now, due to the transmission delay, it is possible that, as what Figure 3.1 shows, the "hit" message arrives earlier at the observer than the "fire" message. If messages are processed upon arrival, i.e., in receive order, and the interval between the two messages is long enough (e.g., greater than the minimum time that human eyes can perceive their inputs), then the observer may experience an illusion that the tank is destroyed before the fighter-jet actually fires at it!

Study of this problem reveals that the "fire" and "hit" messages are causally related and they need to be processed at the observer's site according to the causal order.

Now let us consider another scenario as shown in Figure 3.2. It is a simple DVE which simulates a traffic control system. There are three participants in this DVE: a vehicle, a traffic light that regulates the vehicle's movement and an observer that observes the scenario. Similar to those in Figure 3.1, these three participants are also geographically dispersed and interact with each other by passing messages.

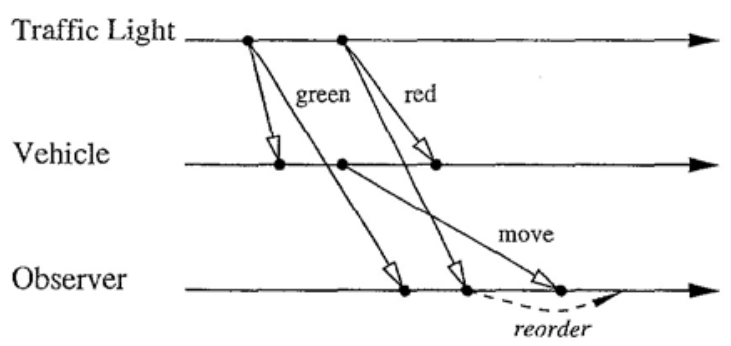

Figure 3.2: Inconsistency caused by incorrect delivery order of concurrent messages

Suppose that the traffic light broadcasts "green" and " red" messages alternately. The vehicle sends either a "move" or a "stop" message to the observer as the response to the received "green" or "red" message. Note that since "red" and "move" are concurrent 
messages, they can be received and processed at the observer's site in any order under the causal order delivery scheme. Due to the transmission delay, as what Figure 3.2 shows, the "red" message probably arrives and is processed at the observer before the vehicle's "move" response to an earlier issued "green" message. Therefore, a kind of inconsistency occurs. The observer will have an illusion that the vehicle tries to move when the traffic light changes from green to red! In fact, in this case, the vehicle strictly obeys the traffic rules and moves only when the traffic light remains green. Obviously, the cause of this inconsistency is the incorrect processing order of certain pair of concurrent messages.

It can be seen from this example that the "green" and "move" messages have a closer relationship than that defined by the "cause-effect" relation. In addition to causal order delivery, receiving of these two messages should not be interrupted by any other “disturbing" messages (e.g., "red" message). Based on this observation, we propose a direct-follow relation to characterize the special relationship between two directly coupled causal messages and a direct-follow order (DFO) delivery scheme to enforce uninterrupted delivery of these messages. The DFO is a stronger ordering mechanism than causal order delivery in the sense that if the former is satisfied, then the latter must also be satisfied. The DFO enforces the delivery order of certain concurrent messages in a way that may sometimes result in conflicts. So, conditions that the delivery order of messages with the direct-follow relation can be ensured are also investigated.

\subsection{Existing Ordering Schemes}

\subsubsection{The Causal Order and Causal Order Delivery}

The inconsistency depicted in Figure 3.1 can be removed by causal delivery of messages. As previously discussed in Section 2.1, a way to characterize the causal order is by using vector time. Applying the rules in Definition 2.1.8, the update of vector time at 
each site is also shown in Figure 3.1. It can be seen that $V T\left(m_{\text {fire }}\right)=[1,0,0]$ and $V T\left(m_{\text {hit }}\right)=[1,2,0]$. Hence it is derivable from Theorem 2.1.1 that $m_{\text {fire }} \rightarrow m_{\text {hit }}$. Both $m_{\text {fire }}$ and $m_{\text {hit }}$ are to be received by the observer. Causal order delivery guarantees that $m_{\text {hit }}$ is processed after $m_{f i r e}$, which is carried out as follow. After $m_{\text {hit }}$ is received by the observer $\left(m_{\text {fire }}\right.$ is still on its way), $V T\left(m_{h i t}\right)$ and $V T\left(P_{\text {observer }}\right)$ are compared. Since the fighter-jet always broadcasts messages to the other two sites, causal order delivery requires that the observer should have processed all the messages from the fighter-jet whose first element is smaller than or equal to $k$ if a message from the tank with its first element of the vector time equal to $k$ is to be processed. This means that processing of $m_{\text {hit }}\left(V T\left(m_{\text {hit }}\right)[0]=1\right)$ is surely withheld until $\operatorname{VT}\left(P_{\text {observer }}\right)[0]$ is updated from 0 to 1 after $m_{\text {fire }}$ has been received and processed.

\subsubsection{Total Order}

It is obvious that the causal order is only a partial order, which captures only messages that have "cause-effect" relations. Causal order delivery places no constraints on delivery order of concurrent messages.

Perhaps the simplest ordering scheme for concurrent messages is the total order [80] proposed by Lamport. A message being sent out is tagged with its sender process's unique identifier. For any pair of concurrent messages, their carried identifers are used to enforce the order ${ }^{3.1}$. However, Lamport's total order is only an artificial and static ordering scheme, thus provides less semantically meaningful ordering among concurrent messages. Total order multicast is widely studied in multicast environments [12, 14, 63], in which local total order and global total order are classified. Local total order only ensures that any two (concurrent) messages having the same multicast group must be processed in the same order by all the processes in that group. Global total order defines

\footnotetext{
${ }^{3.1}$ Note that any pair of messages from the same sender process can never be concurrent, thus concurrent messages cannot carry identical identifiers.
} 
a stronger property that any two (concurrent) messages, as long as they have the same destination processes, must be processed in the same order at these processes.

Refer back to Figure 3.2, if there are multiple observers receiving messages from the traffic light and the vehicle, global total order ensures that the concurrent "move" and "red" messages are processed in the same order, which results in an identical view (no matter correct or incorrect), at all the observers. However, the correct order may not be enforced.

\subsubsection{Critical Causality and Causal-Receive Order Delivery}

A closely-related work to address the inconsistency problem shown in Figure 3.2 is the notion of critical causality (similar to Definition 3.3.1) and causal-receive order delivery proposed in [154]. Instead of providing a delivery scheme stronger than causal order delivery, causal-receive order delivery resorts to a weaker scheme. In addition to processing messages in receive order without any constraints, the only ordering requirement to be fulfilled is the processing order of messages that have critical causal relation ${ }^{3.2}$, e.g., the "green" message and the "move" message. However, as reported, even this ordering requirement is not easy to be ensured. Using the algorithm in [154], in some rare cases, the processing order of critical causal related messages are still possibly violated.

\subsection{Direct-Follow Relation}

The ,Jrmalization hereafter is based on the terms and definitions introduced in Section 2.1. To characterize the directly coupled causal messages, the following relation is defined:

\footnotetext{
${ }^{3.2}$ Note that messages having critical causal relation are generally application dependent. Not all causally related messages are defined as having critical causal relation.
} 
Definition 3.3.1 (direct-follow relation $\stackrel{d}{\Rightarrow}$ ). Given the standard enumerations of $E_{\mathrm{i}}$ and $E_{\mathrm{j}}$, which occur at processes $P_{i}$ and $P_{\mathrm{j}}$ respectively $(i \neq j)$, if a pair of messages $m$ and $m^{\prime}$ meet the following criteria:

1. $S_{m} \in E_{i}$;

2. $R_{m}, S_{m^{\prime}} \in E_{j}$; and

3. $S_{m^{\prime}}$ is the event immediately after $R_{m}$ in $E_{j}$.

a relationship, namely direct-follow, is defined upon $m$ and $m^{\prime}$, denoted as $m \stackrel{\text { d }}{\Rightarrow} m^{\prime} . m$ and $m^{\prime}$ are called a pair of direct-follow messages.

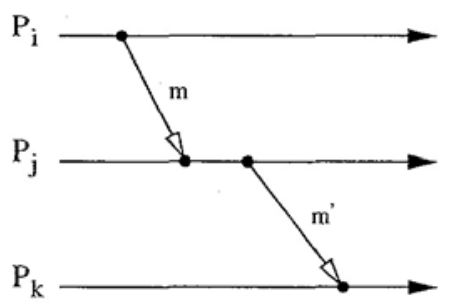

Figure 3.3: Direct-follow messages

Figure 3.3 further illustrates this definition. Note that we do not consider internal events in standard enumerations in this chapter. If $m$ and $m^{\prime}$ are a pair of direct-follow messages, there should not be any send or receive events between $R_{m}$ and $S_{m^{\prime}}$.

Based on Definition 3.3.1, we have the following lemmas which specify the properties of the direct-follow relation and direct-follow messages.

Lemma 3.3.1. The direct follow relation is non-transitive.

PROOF (by contradiction):

Assume $\mathrm{m}_{1} \stackrel{\mathrm{d}}{\Rightarrow} m_{2}$ and $m_{2} \stackrel{\mathrm{d}}{\Rightarrow} m_{3}$. If $\stackrel{\mathrm{d}}{\Rightarrow}$ is transitive, then we have $m_{1} \stackrel{\mathrm{d}}{\Rightarrow} m_{3}$. According to criterion 3 of Definition 3.3.1, $S_{m_{3}}$ should occur immediately after both $R_{m_{1}}$ and $R_{m_{2}}$ 
at a process. This is impossible and thus the assumption that the direct-follow relation is transitive is incorrect.

Lemma 3.3.2. If $m \stackrel{\text { d }}{\Rightarrow} m^{\prime}$, then $S_{m} \rightarrow S_{m^{\prime}}$.

\section{PROOF:}

It is obvious from Definition 3.3.1 that $S_{m} \rightarrow R_{m}$ and $R_{m} \rightarrow S_{m^{\prime}}$ hold. Since $\rightarrow$ is transitive, we have $S_{m} \rightarrow S_{m^{\prime}}$.

For a given event, its local predecessor set consists of events that occur before it and its local successor set consists of events that occur after it.

Definition 3.3.2 (local predecessor set $\mathcal{P}_{l}$ and successor set \&). Given the standard enumeration of $E_{i}=\left\{e_{i 1}, e_{i 2}, \ldots, e_{i n}, \ldots\right\}$ which occurs at process $P_{i}$, the local predecessor set of event $e_{i n}$, denoted as $\mathcal{P}_{l}\left(e_{i n}\right)$, is defined as a subset of $E_{i}$ where $\mathcal{P}_{l}\left(e_{i n}\right)=\left\{e_{i k} \mid k<n\right\}$. The local successor set of event $e_{i n}$, denoted as $\mathcal{S}_{l}\left(e_{i n}\right)$, is defined as a subset of $E_{i}$ where $\mathcal{S}_{l}\left(e_{i n}\right)=\left\{e_{i k} \mid k>\mathbf{n}\right\}$.

Given the above definition, we then have the following property of direct-follow messages:

Lemma 3.3.3. Suppose that $m \stackrel{d}{\Rightarrow} m^{\prime}, S_{m} \in E_{i}, R_{m}$ and $S_{m^{\prime}} \in E_{j}$.

1. $\forall x, x \neq m$, if $S_{x} \in E_{i}$ and $S_{x} \| S_{m^{\prime}}$, then $S_{x} \in \mathcal{S}_{l}\left(S_{m}\right)$.

2. $\forall x, x \neq m^{\prime}$, if $S_{x} \in E_{j}$ and $S_{x} \| S_{m}$, then $S_{x} \in \mathcal{P}_{l}\left(R_{m}\right)$.

PROOF (by contradiction):

1. Because of $x \neq m$ and $S_{x}, S_{m} \in E_{i}$, either $S_{m} \mathcal{E}_{s} \mathcal{B}_{l}\left(S_{m}\right)$ or $S_{m} \in \mathcal{S}_{l}\left(S_{x}\right)$ must hold. Assume $S_{m} \in \mathcal{S}_{l}\left(S_{x}\right)$ holds. From Definition 3.3.2, we have $S_{x} \rightarrow S_{m}$. From Lemma 3.3.2, we thus have $S_{x} \rightarrow S_{m^{\prime}}$. This is contradictory with $S_{x} \| S_{m^{\prime}}$. 
2. Because of $x \neq m$ and $S_{x}, R_{m} \in E_{j}$, either $S_{x} \in \mathcal{P}_{l}\left(R_{m}\right)$ or $R_{m} \in \mathcal{P}_{l}\left(S_{x}\right)$ must hold. Assume $R_{m} \in \mathcal{P}_{l}\left(S_{x}\right)$ holds. From Definition 3.3.2, we have $R_{m} \rightarrow S_{x}$. Because of $S_{m} \rightarrow R_{m}$ (the causal relation), we thus have $S_{m} \rightarrow S_{x}$. This is contradictory with $S_{x} \| S_{m}$

The above lemma states that for a pair of direct-follow messages, $m$ and $m$ ' $\left(S_{m} \in\right.$ $E_{i}, R_{m}$ and $S_{m^{\prime}} \in E_{j}$ ): (1)Any message sent by process $P_{i}$, which is concurrent with $m$ ', must be sent after $m$; and (2) Any message sent by process $P_{j}$, which is concurrent with $m$, must have been sent before $m$ is received.

\subsection{Direct-Follow Order Delivery}

To guarantee uninterrupted delivery of directly coupled messages, the following message delivery order is defined:

Definition 3.4.1 (direct-follow order delivery). Given a pair of direct-follow messages $m \stackrel{\mathrm{d}}{\Rightarrow} m^{\prime}\left(S_{m} \in E_{i}, R_{m}\right.$ and $\left.S_{m^{\prime}} \in E_{j}, i \neq j\right), m$ and $m^{\prime}$ are processed according to the direct-follow order if at any process $P_{k}(k \neq i, k \neq j)$ which receives them, $m$ and $m i$ are processed not only in causal order but also in an uninterrupted manner, i.e., any other message $\mathrm{x}$, which is also sent to $\mathrm{P}_{k}$ and satisfies one of the following conditions, cannot be processed between them:

1. $S_{x} \in E_{i}$ and $S_{x} \| S_{m^{\prime}}$

2. $S_{x} \in E_{j}$ and $S_{x} \| S_{m}$

The philosophy behind direct-follow order (DFO) delivery is further depicted in Figure 3.4, where $x_{1}$ and $x_{2}$ satisfy conditions (1) and (2) respectively as described in Definition 3.4.1. $S_{x_{1}}$ is a local successor of $S_{m}$, thus $x_{1}$ may carry different state information 
of $P_{i}$. If $x_{1}$ is processed by process $P_{k}$ before $m$ ', $P_{k}$ may interpret it as the "cause" of $m$ '. Similarly, $S_{x_{2}}$ is a local predecessor of $S_{m^{\prime}}$. If $x_{2}$ is processed by process $P_{k}$ after $m, P_{k}$ may interpret it as the "effect" of $m$. Thus, to maintain the correct "cause" and "effect" of directly coupled causal messages, $x_{1}$ and $x_{2}$ cannot be processed between $m$ and $m$ at process

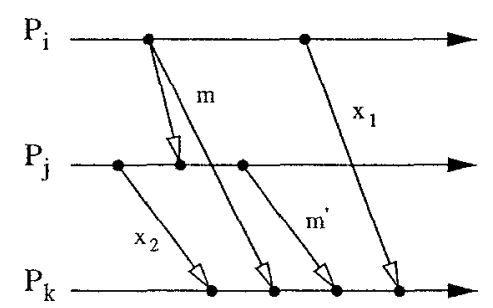

Figure 3.4: Direct-follow order delivery

Note that only concurrent messages originating from the senders of $m$ and $m$ ' are considered as "disturbing" messages, since these are the messages that may cause incorrect interpretation of "cause" and "effect" at a receiving process.

Lemma 3.4.1. Suppose that $m \stackrel{\mathrm{d}}{\Rightarrow} m^{\prime}\left(S_{m} \in E_{i}, R_{m}\right.$ and $\left.S_{m^{\prime}} \in E_{j}\right)$ and that $m$ and $m$ ' are processed according to the DFO. Given a message $x$, at any process $P_{k}(k \neq i, j)$ which receives messages $m, m^{\prime}$ and $x$,

1. If $S_{x} \in \mathcal{S}_{l}\left(S_{m}\right)$, then $R_{m^{\prime}} \rightarrow R_{x}$ must hold at $P_{k}$.

2. If $S_{x} \in \mathcal{P}_{l}\left(S_{m^{\prime}}\right)$, then $R_{x} \rightarrow R_{m}$ must hold at $P_{k}$.

\section{PROOF:}

1. Because of $m \stackrel{\text { d }}{\Rightarrow} m^{\prime}$ and $S_{x} \in \mathcal{S}_{l}\left(S_{m}\right)$, we have either $S_{x} \| S_{m^{\prime}}$ or $S_{m^{\prime}} \rightarrow S_{x}$ $\left(S_{x} \rightarrow S_{m^{\prime}}\right.$ is not possible because $S_{x} \in \mathcal{S}_{l}\left(S_{m}\right)$ and $R_{m}$ is immediately followed by $S_{m^{\prime}}$ at $P_{j}$ ). If $S_{x} \| S_{m^{\prime}}$, since $m$ and $m^{\prime}$ are processed according to the DFO, either $R_{x} \rightarrow R_{m}$ or $R_{m^{\prime}} \rightarrow R_{x}$ must hold (see Definition 3.4.1). $S_{m} \rightarrow S_{x}$ because 
of $S_{x} \in \mathcal{S}_{l}\left(S_{m}\right)$. So, to causally deliver $m$ and $x, R_{m} \rightarrow R_{x}$ must be true and thus we have $R_{m^{\prime}} \rightarrow R_{x}$. If $S_{m^{\prime}} \rightarrow S_{x}$, it is obvious that $R_{m^{\prime}} \rightarrow R_{x}$ must hold for causal order delivery.

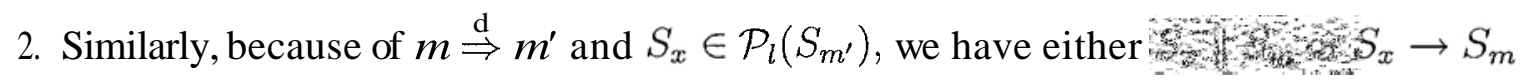
$\left(S_{m} \rightarrow S_{x}\right.$ is not possible because $S_{x} \in \mathcal{P}_{l}\left(S_{m}^{\prime}\right)$ and $R_{m}$ is immediately followed by $S_{m^{\prime}}$ at $P_{j}$ ). If $S_{x} \| S_{m}$, since $m$ and $m^{\prime}$ are processed according to the DFO, either $R_{x} \rightarrow R_{m}$ or $R_{m^{\prime}} \rightarrow R_{x}$ must hold (see Definition 3.4.1). $S_{x} \rightarrow S_{m^{\prime}}$ because of $S_{x} \in \mathcal{P}_{l}\left(S_{m^{\prime}}\right)$. So, to causally deliver $m^{\prime}$ and $x, R_{x} \rightarrow R_{m^{\prime}}$ must be true and thus we have $R_{x} \rightarrow R_{m}$. If $S_{x} \rightarrow S_{m}$, it is obvious that $R_{x} \rightarrow R_{m}$ must hold for causal order dekvery.

The DFO effectively enforces the delivery order of certain concurrent messages. The following lemma further elaborates this property.

Lemma 3.4.2. Suppose that $m \stackrel{\mathrm{d}}{\Rightarrow} m^{\prime}\left(S_{m} \in E_{i}, R_{m}\right.$ and $\left.S_{m^{\prime}} \in E_{j}\right)$ and that $m$ and $m$ are delivered according to the DFO. Given a message $x$, at any process $P_{k}(k \neq i, j)$ which receives messages $m, m^{\prime}$ and $x$,

1. If $S_{x} \in E_{i}$ and $S_{x} \| S_{m^{\prime}}$, then $R_{m^{\prime}} \rightarrow R_{x}$ must hold at $P_{k}$.

2. If $S_{x} \in E_{j}$ and $S_{x} \| S_{m}$, then $R_{x} \rightarrow R_{m}$ must hold at $P_{k}$.

\section{PROOF:}

It is obvious from lemmas 3.3.3 and 3.4.1.

\subsection{Resolving Conflicts}

It should be noticed that direct-follow messages may not always be able to be processed according to the DFO. The causal order delivery is strictly preserved in a distributed 
system for any pair of messages which are causally related. In addition, as indicated in Lemma 3.4.2, the DFO introduces constraints on the delivery order of concurrent messages. This may in turn lead to conflicts in message dependency.

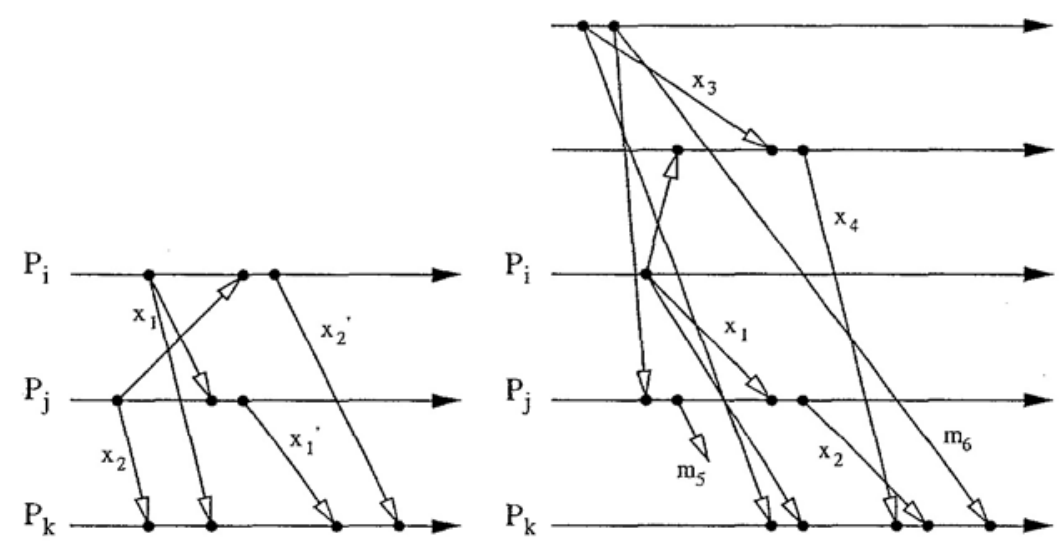

(a)

(b)

Figure 3.5: Conflicts in the DFO

Two examples are illustrated in Figure 3.5. In Figure 3.5(a), $x_{1} \stackrel{d}{\Rightarrow} x_{1}{ }^{\prime}$ and $x_{2} \stackrel{d}{\Rightarrow} x_{2}{ }^{\prime}$. However, the DFO delivery of the two pairs of direct-follow messages, $x_{1}$ and $x_{1}{ }^{\prime}$ and $x_{2}$ and $x_{2}{ }^{\prime}$, cannot be both guaranteed, because the former requires $R_{x_{2}} \rightarrow R_{x_{1}}$ at $P_{k}$ while the latter requires $R_{x_{1}} \rightarrow R_{x_{2}}$ at $P_{k}$. This problem is solved if one of the pairs is not delivered according to the DFO.

Figure 3.5(b) presents a more complex situation. Suppose that $x_{1} \stackrel{d}{\Rightarrow} x_{2}$ and $x_{3} \stackrel{d}{\Rightarrow} x_{4}$. Without message $m_{5}$, a valid message processing order, i.e., $\left\{R_{x_{3}}, R_{x_{1}}, R_{x_{4}}, R_{x_{2}}, R_{m_{6}}\right\}$, exists at $P_{k}$, which not only preserves causal order delivery, but also guarantees that the two pairs of direct-follow messages are both processed according to the DFO. However with $m_{5}$, such a valid message processing order does not exist any more. On one hand, the DFO delivery of $x_{1}$ and $x_{2}$ requires that $R_{m_{5}} \rightarrow R_{x_{1}}$ The causal order delivery also requires that $R_{m_{6}} \rightarrow R_{m_{5}}$ and $R_{x_{1}} \rightarrow R_{x_{4}}$. So, we have $R_{m_{6}} \rightarrow R_{x_{4}}$. On the other hand, the DFO delivery of $x_{3}$ and $x_{4}$ requires that $R_{x_{4}} \rightarrow R_{m_{6}}$. This problem is due to the existence of $m_{5}$, which adds an extra causal relation and prevents the DFO delivery of 
both pairs of the direct-follow messages. Similarly, it is solved if one of the pairs is not delivered according to the DFO.

Further study reveals that both the above situations are caused by the conflicts in message dependency. In general, the causal dependency between messages is characterized by the causal relation (see Definition 2.1.3 and Definition 2.1.9). However, if two concurrent messages must be processed according to a certain order at a process, an additional message dependency is required over and above the causal dependency. ${ }^{3.3}$ As stated in Lemma 3.4.2, the DFO delivery of direct-follow messages imposes a delivery order on some concurrent messages and thus introduces those additional dependencies.

Definition 3.5.1 (dependency set $D$ ). The dependency set of the message $m, D(m)$, is defined recursively as follows:

1. For any message $x, S_{x} \| S_{m}$, if $x$ is semantically required to be delivered before $m$ at a process, $m$ is said to be dependent on $x$, denoted as $x \in \mathcal{D}(m)$;

2. For any message $x$, if $S_{x} \rightarrow S_{m}, x \in \mathcal{D}(m)$; and

3. If message $m^{\prime} \in \mathcal{D}(m)$, for any message $x \in \mathcal{D}\left(m^{\prime}\right), m$ also depends on $x$ (i.e., $x \in \mathcal{D}(m))$.

The DFO delivery of direct-follow messages can be guaranteed if and only if there does not exist a cyclic message dependency. The theorem is given below.

Theorem 3.5.1. Given a pair of direct-follow messages $m \stackrel{\mathrm{d}}{\Rightarrow} m^{\prime}\left(S_{m} \in E_{i}, R_{m}\right.$ and $\left.S_{m^{\prime}} \in E_{j}, i \neq j\right)$, they can be processed in the DFO iff:

$$
\text { 1. } \nexists x\left(\left(S_{x} \in E_{i}\right) \wedge\left(S_{x} \| S_{m^{\prime}}\right) \wedge\left(x \in \mathcal{D}\left(m^{\prime}\right)\right)\right) ; \text { and }
$$

\footnotetext{
${ }^{3.3}$ Note that concurrent messages can be processed in any order at a process. There exists a dependency between two concurrent messages only when there is a processing order enforced on the messages at a receiving process.
} 
2. $\nexists x\left(\left(S_{x} \in E_{j}\right) \wedge\left(S_{x} \| S_{m}\right) \wedge(m \in \mathcal{D}(x))\right)$

\section{PROOF}

- We first prove, by contradiction, the necessity (i.e., if $m$ and $m$ ' can be processed according to the DFO, both conditions must be true). Assume one of the condition is not true, e.g., the first condition. So, $\exists x\left(\left(S_{x} \in E_{i}\right) \wedge\left(S_{x} \| S_{m^{\prime}}\right) \wedge\left(x \in \mathcal{D}\left(m^{\prime}\right)\right)\right)$. According to Definition 3.5.1, $x \in \mathcal{D}\left(m^{\prime}\right)$ implies $x$ must be processed before $m$ '. Since $m$ and $m$ can be delivered according to the DFO, from Lemma 3.4.2 we have $R_{m^{\prime}} \rightarrow R_{x}$. Thus, there is a contradiction. A similar argument can be applied to the second condition.

- We then prove, by contradiction again, the sufficiency (i,e., if both conditions are true, $m$ and $m$ must be able to be processed according to the DFO). If $m$ and $m$ ' cannot be processed according to the DFO, there are two cases to consider according to Definition 3.4.1:

- There is a message $x$ which satisfies the condition $\left(S_{x} \in E_{i}\right) \wedge\left(S_{x} \| S_{m^{\prime}}\right)$ and there exists a process $P_{k}$ so that $x$ has to be delivered before $m^{\prime}$ at $P_{k}$. Thus, according to Definition 3.5.1, $x \in \mathcal{D}\left(m^{\prime}\right)$. This contradicts the first condition of the theorem.

- There is a message $x$ which satisfies the condition $\left(S_{x} \in E_{j}\right) \wedge\left(S_{x} \| S_{m}\right)$ and there exists a process $P_{k}$ so that $x$ has to be delivered after $m$ at $P_{k}$. Thus, by Definition 3.5.1, $m \in \mathcal{D}(x)$. This contradicts the second condition of the theorem. 


\subsection{Summary}

The concept of event ordering is fundamental to applications running on a distributed system. Inconsistency problems which may arise in such applications are directly related to the event ordering. So far, the causal order has been widely studied and implemented to provide necessary consistency. However, the order in which concurrent messages are processed, which cannot be guaranteed by the causal order, may still cause semantically inconsistencies.

In general, there is no intrinsic mechanism for ordering concurrent messages. As discussed before, existing total order delivery schemes cannot solve the inconsistency problem illustrated in Figure 3.2. For Lamport's total order, it is impossible to find a unique sequence of process identifiers that satisfies every ordering need of concurrent messages that is required in order to process directly coupled causal messages in an uninterrupted manner. Causal and Totally Ordered Communication Support (CATOCS [14]) extends the causal order to guarantee that concurrent messages will be delivered to all the processes receiving them in the same order, but this order may not be the semantically correct one that we want.

The debate on ordering semantics of CATOCS in the context of multicast protocols drew much attention in the mid '90s. Cheriton and Skeen [28] criticize the applicability of CATOCS for several classes of distributed applications. Birman [11] responds by describing a significant range of applications where ordered communication provides tangible benefits such as fault tolerance and a simplified programming model. However, the criticism that the causal order cannot enforce some necessary semantic ordering constraints remains.

In our work, we proposed a new relation, i.e., direct-follow relation, to characterize directly coupled causal messages and a corresponding message delivery scheme, i.e., the 
DFO delivery. The DFO delivery effectively enforces the delivery order of certain concurrent messages and thus can be used to eliminate inconsistencies caused by these messages. However, messages with the direct-follow relation cannot be always delivered according to the DFO because of the conflicts in message dependency introduced by the DFO. We further explored the properties of the direct-follow relation and gave the conditions under which direct-follow messages can be delivered according to the DFO.

The major concern of the implementation of the DFO is the way to determine the delivery order of concurrent messages. One approach is to use application specific information to detect direct-follow messages. Using the example in Figure 3.4, suppose all pairs of direct-follow messages $m$ and $m^{\prime}$ are pre-defined in the application. The message $m$ can then carry extra information about its direct-follow message $m$ '. When $m$ arrives at $P_{k}$, its delivery is delayed until $m^{\prime}$ arrives. The normal causal information that the causal order delivery algorithm appends to $m^{\prime}$ can then be used to ensure that $m$ is not processed before any message $x$ such that $S_{x} \in \mathcal{P}_{l}\left(R_{m}\right)$ (e.g., $\left.x_{2}\right)$. Similarly, the normal causal information that is appended to any message $x$ such that $S_{x} \in \mathcal{S}_{l}\left(S_{m}\right)$ (e.g., $\left.x_{1}\right)$ can be used to ensure that $x$ is not processed until after both of the directly-coupled messages $m$ and $m^{\prime}$ have been processed.

Note that conflicts can still arise with this algorithm. For example, in Figure 3.5(a), the causality information appended to $x_{1}^{\prime}$ will indicate that $x_{1}$ cannot be delivered before $x_{2}$ and similarly the causality information appended to $x_{2}^{\prime}$ will indicate that $x_{2}$ cannot be delivered before $x_{1}$. However, this conflict can be detected locally at process $P_{k}$, and an application specific decision can be made to resolve the conflict.

The implementation of the DFO for a more general case is more difficult. Again with reference to Figure $3.4, m^{\prime}$ and $x_{1}$ are concurrent messages. Assume that $x_{1}$ arrives at $P_{k}$ first. If $P_{k}$ does not have knowledge on whether or not $P_{j}$ sends message $m^{\prime}$, it cannot decide whether or not to process $x_{1}$.So, for a destination process of a multicast 
Ph.D Thesis

CHAPTER 3.

message, it has to know whether or not it may receive a direct-follow message from any other process in the destination set of the same multicast message. A possible approach is to introduce additional protocol messages among the processes. For the example in Figure 3.4, if $m$ does not result in a direct-follow message at $P_{j}$, this information has to be carried either on message $m$ itself or $P_{j}$ has to send a protocol message to $P_{k}$ after receiving $m$, thus $P_{k}$ can make a decision on whether or not to process message $x_{1}$. 


\section{Chapter 4}

\section{TWSK, An Extensible Framework for Time Warp Simulation Kernel}

\subsection{Introduction and Motivations}

Compared to sequential and conservative simulations, the complex nature, particularly the synchronization semantics, of Time Warp simulation makes its design and implementation much more difficult. To ease the development of various Time Warp simulation applications and to separate simulation logic from the Time Warp mechanisms, a usual approach is to divide Time Warp simulation into two parts as shown in Figure 4.1 [59].

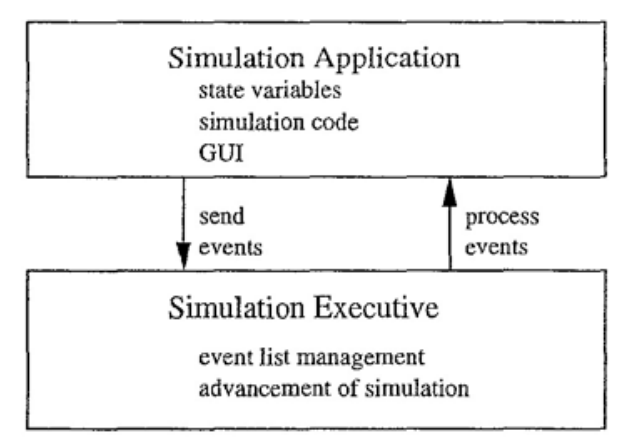

Figure 4.1: Two levels of Time Warp simulation

The lower part is simulation executive, which maintains data structures, e.g., event list and simulation time variable, and realizes Time Warp synchronization semantics, e.g., 
rollbacks and GVT approximation, that are common to and usable for all simulation applications. Commercial vendors often commercializes simulation executives as "general purpose" products that can be used for building up various simulation applications. The upper part is simulation application, which makes use of simulation executive and implements real simulation scenarios to meet particular simulation goals. The implementation of a simulation application normally involves three tasks: choosing a suitable collection of state variables to represent the state of the simulation, coding the simulation behavior and handling users' input/output.

It can be seen that this levelized approach effectively makes the development of these two parts independent from each other. A simulation executive encapsulates Time Warp mechanism well inside and provides necessary interfaces to simulation applications. A simulation application makes use of these interfaces, thus, avoids the involvement of low level details. For example, there are two methods in the provided interface, send () and process (), as shown in Figure 4.1. send() is called when the application is to send a message with the scheduled event to any other LP and process () is called when the application is notified to process a received event.

Our research on Time Warp simulation follows this approach. For the purpose of introducing new mechanisms and conducting performance study of them using various benchmarking models, it is desirable to have multiple simulation executives running with our proposed mechanisms provide identical interfaces to the simulation applications. This motivates us to establish an extensible framework that is easy to be extended to create different simulation executives and directly used by simulation applications. In the following sections, the design rationale and implementation issues of our developed framework, namely, Time Warp simulation kernel (TWSK), will be elaborated further. 


\subsection{Architecture of TWSK}

An architectural view of TWSK is shown in Figure 4.2, in which a simulation executive consists of three layered parts, which are explained below:

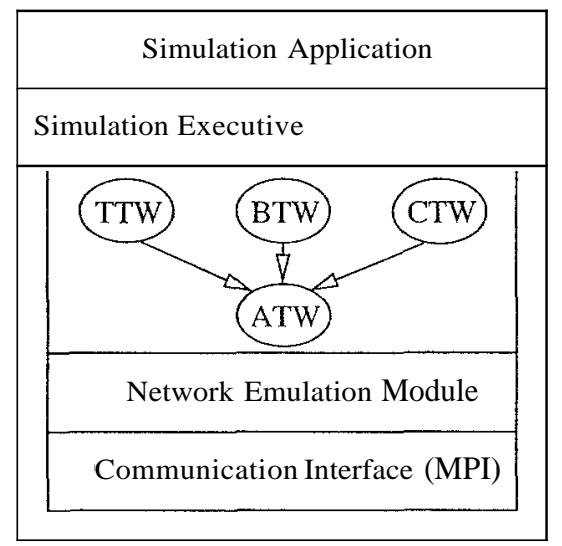

Figure 4.2: An architectural view of FWSK

- Communication Interface: Communication interface provides the functionalities of buffering, sending and receiving messages from the underlying communication system. Currently, we adopt MPICH, a portable message passing interface standard (MPI) [53] implementation, as the basis for the implementation of the interface for several reasons: (1)MPICH provides a free of charge, general purpose message passing library that hides low level detail of implementing communication amongst networked computing nodes; (2) MPICH is portable to a wide range of platforms (including shared-memory machines, loosely-coupled workstation clusters and even individual PCs), thus the usage of MPICH enables TWSK to be running on various environments; and (3) MPICH provides barrier synchronization, which is handy to be used to synchronize the start and stop of a simulation.

- Network Emulation Module: The communication interface built on MPICH can only provide FIFO transfer of messages. Although FIFO channels are normally as- 
sumed by most Time Warp simulations, Time Warp is also workable with non-FIFO channels. In order to conduct the performance evaluation of existing mechanisms and our proposed mechanisms in a WAN environment or using non-FIFO communication channels, an optional network emulation module is introduced into the kernel. This module is tunable by adjusting the rate of out-of-order messages and the delay of messages going through a communication link so that an emulated WAN can be obtained.

- Time Warp Module: On top of the communication interface and the network emulation module, the time warp module forms the major part of a simulation executive. As mention earlier; it is required to have multiple executives running with different synchronization mechanisms. This motivates us to seek an objectoriented approach to design the module. As shown in the figure, we first abstract Time Warp mechanism into a collection of abstract classes, namely, Abstract Time Warp (ATW), and then derive from ATW by overriding necessary functions to obtain three different categories of Time Warp modules. The Traditional Time Warp (TTW) implements a basic Time Warp simulation mechanism with no optimization. The Batch Time Warp (BTW) reuses TTW implementation but replaces the traditional per-event based cancellation mechanism with our proposed batch based cancellation mechanism. The Causal Time Warp (CTW) implements causal order based Time Warp (COBTW). The mechanisms of BTW and CTW will be elaborated in Chapter 5 and Chapter 6 respectively.

\subsection{Class View of Abstract Time Warp}

Introducing ATW is to provide two key features, namely, extensibility and reusability, in the development of Time Warp simulations in order to simplify the development process. 
ATW formalizes the Time Warp mechanisms as an open framework, which consists of a collection of interfaces (having only pure virtual functions) and derivable classes, as shown in Figure 4.3.

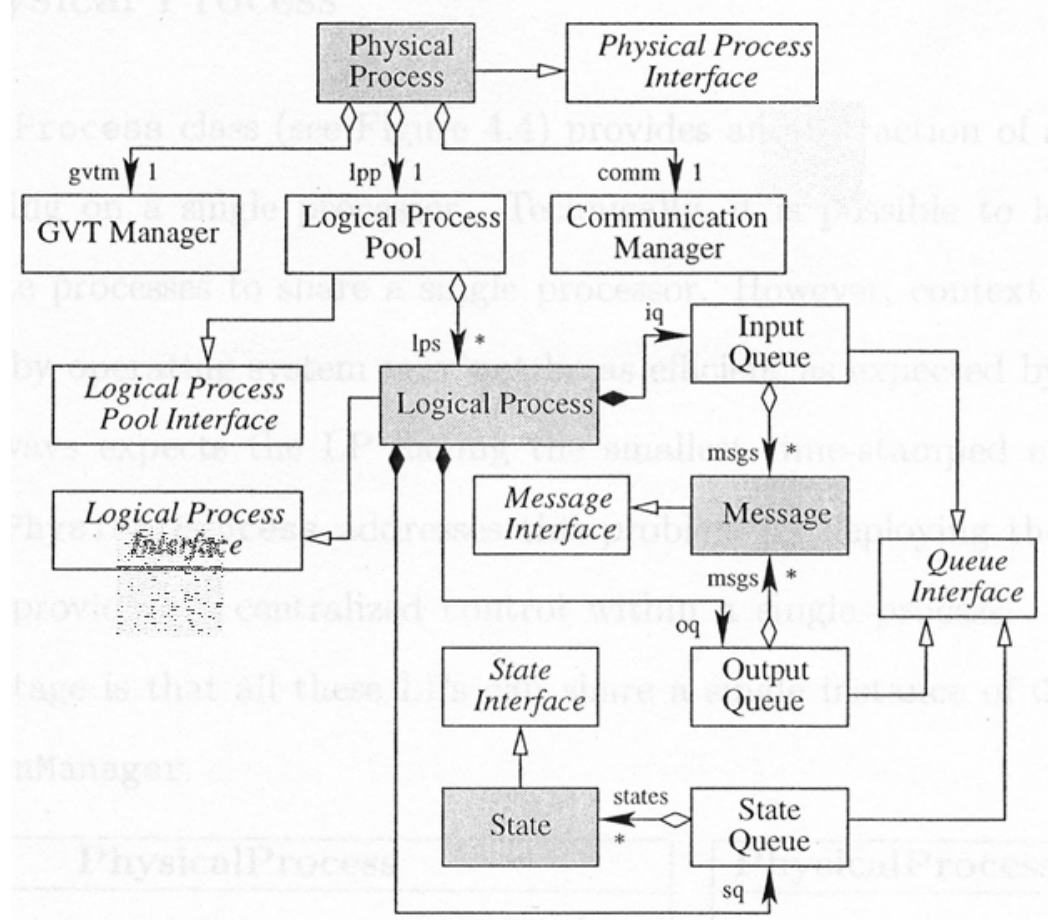

Figure 4.3: Class diagram of ATW

Before going into details of each class, two roles should be distinguished in using ATW. Executive developer who designs a new executive is required to derive all abstract classes ${ }^{4.1}$ with full implementation of most pure functions of corresponding interfaces which are supposed to be the minimal mutable parts of any executive. Since other member functions in ATW, which are supposed to be common to most of executives, are designed to be working with the pure functions in interfaces, they are generally reusable in the derived classes in the new executive. Only a small number of classes (PhysicalProcess, LogicalProcess, Message and State shown in shaded boxes) in an executive are visible to application developers. By design, these classes are required to be further derived by

\footnotetext{
${ }^{4.1}$ The abstract classes are those derived from interface classes (class name shown in italics), e.g., class Physical Process.
} 
application developers, based on the ones provided by executive developers, to incorporate simulation logic.

\subsubsection{Physical Process}

The PhysicalProcess class (see Figure 4.4) provides an abstraction of a simulation executable running on a single processor. Technically, it is possible to let multiple LPs run as separate processes to share a single processor. However, context switch between LPs provided by operating system may not be as efficient as expected by simulations (a simulation always expects the LP having the smallest time-stamped event to be executed next). PhysicalProcess addresses this problem by deploying these LPs into an LP pool and providing a centralized control within a single process. In addition, an obvious advantage is that all these LPs can share a single instance of GVTmanager and CommunicationManager.

\begin{tabular}{|l|}
\hline \multicolumn{1}{|c|}{ PhysicalProcess } \\
\hline int size \\
int ppid \\
vector< pair<int, int> > map \\
GVTManager* gvtm \\
LogicalProcessPool* Ipp \\
CommunicationManager* comm \\
\hline void reg_msg(int type, ALLOCATOR alloc) \\
void reg_lp(LogicalProcess* lp) \\
void reg_all() \\
void run() \\
\hline
\end{tabular}

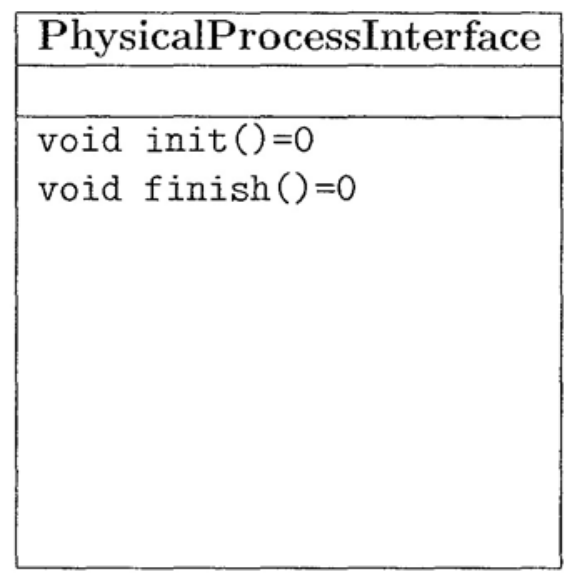

Figure 4.4:PhysicalProcess Class and PhysicalProcessInterface Class

Derived executives or applications generally need to introduce their own messages. The management of these messages is generalized in CommunicationManager and exposed to executive/application developers by reg_msg(). For any message that is going to be transmitted in the simulation, it must be registered with the executive us- 
ing reg_msg () (more details are discussed in Section 4.3.4). reg_lp() and reg_all() are used to assist LP deployment. Each call of reg_lp() registers a local LP with the physical process. Then reg_all () is made to gather a view of the deployment of all the LPs in the simulation. This information is locally stored in map and used by the CommunicationManager throughout the simulation (more details are discussed in Section 4.4.1). The PhysicalProcessInterface class (see Figure 4.4) has two members, init() and finish(), which enable executive/application developers to invoke customized initialization and clean-up code at time the simulation starts and ends.

\subsubsection{Logical Process Pool}

All the LPs managed by an instance of PhysicalProcess class is represented by lps in the LogicalProcessPool class (see Figure 4.5). over() is used to test if all the LPs have finished simulating events. During the simulation, schedule() is called, which scans through lps to find out the LP having the smallest time-stamped event to execute the next. $g_{\mathrm{c}}\left(\mathrm{)}\right.$ does garbage collection by calling each LP's $\mathrm{gc}_{\mathrm{C}}(\mathrm{)}$. Similarly, the LogicalProcessPoolInterface class (see Figure 4.5) has init() and finish(), which allow executive developers' code to be called at time the simulation starts and ends.

\begin{tabular}{|l|}
\hline \multicolumn{1}{|c|}{ LogicalProcess Pool } \\
\hline vector< LogicalProcess* $>$ IPS \\
\hline bool over() \\
LogicalProcess* schedule \\
void gc(SIMTIME st)
\end{tabular}

LogicalProcessPoolInterface
void init ()$=0$
void $\operatorname{inish}()=0$

Figure 4.5: LogicalProcessPool Class and LogicalProcessPoolInterface Class

\subsubsection{Logical Process}

The LogicalProcess class (see Figure 4.6) is essentially an abstract of LP in Time Warp, whose basic elements are expressed as data members: lvt locally stores current simula- 


\begin{tabular}{|l|}
\hline \multicolumn{1}{|c|}{ LogicalProcess } \\
\hline int lpid \\
SIMTIME lvt \\
State* state \\
InputQueue iq \\
OutputQueue oq \\
StateQuene sq \\
\hline bool over() \\
void gc(SIMTIME st) \\
void chkpt_state() \\
void recover_state(SIMTIME st) \\
Message* run() \\
\hline
\end{tabular}

\begin{tabular}{|l|}
\hline \multicolumn{1}{|c|}{ LogicalProcessInterface } \\
\hline void init()=0 \\
void process (Message* msg) $=0$ \\
void finish ()$=0$ \\
void send (Message* msg) $=0$ \\
void recv (Message* msg) $=0$ \\
\\
\\
\end{tabular}

Figure 4.6: LogicalProcess Class and LogicalProcessInterf ace Class

tion time of the LP; state points to an instance of a State derived class that wraps all the user-defined state variables; iq, oq and sq represent the LP's input queue, output queue and state queue respectively. For the purpose of checkpointing the LP's current state and recovering from an old state, chkpt_state() and restore_state() are provided. chkpt_state() pushes the current state at the back of sq. restore_state() searches through sq reversely and recovers the LP from the latest saved state that has the timestamp smaller than the requested value. Note that the choice of frequent state saving or infrequent state saving is determined by the frequency of calling chkpt_state(). By overriding chkpt_state() and restore_state(), it is possible to implement incremental state saving. run( ) rolls back the LP if necessary to guarantee the local causality and retrieves the next unprocessed message for processing. The LogicalProcessInterface class (see Figure 4.6) has five members. send() and recv() must be implemented by executive developers to perform specific operations upon sending and receiving any message. process () is required to be overridden by executive/application developers to supply executive/simulation specific handling as well. process () generalizes the event flow from the executive to the application as shown in Figure 4.1 whereas imposes no limitation on application code. Multiple events can be scheduled within a single run of 
process () and instantaneous response (zero processing time) is allowed. All the scheduled events are to be transferred through the underlying network momentarily by the communication manager as described in Section 4.4.2. An example implementation of process () in PHOLD simulation can be found in Appendix A.

\subsubsection{Message}

\begin{tabular}{|l|}
\hline \multicolumn{1}{|c|}{ Message } \\
\hline int type \\
bool positive \\
SIMTIME st \\
int color \\
int send_lp \\
vector int $>$ recv_lps \\
\hline
\end{tabular}

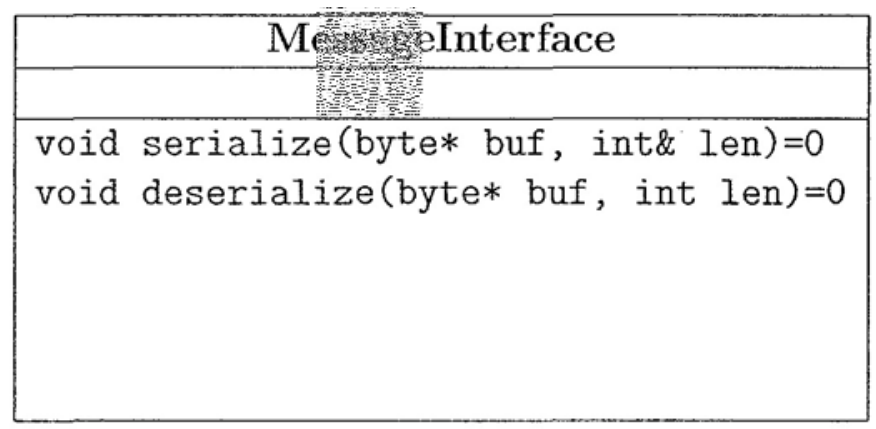

Figure 4.7: Message Class and MessageInterface Class

The Message class (see Figure 4.7) is designed to be the base class of all the messages introduced by executive developers and application developers in simulations. Any derived message has a unique type identifier and is associated with a particular allocator. An allocator is of type ALLOCATOR which is type defined as Message* (ALLOCATOR*) (), i.e., a function allocating storage for the message. After the application developer registers all the user-defined simulation messages with the executive using PhysicalProcess: :reg_msg() and makes the allocators known to the CommunicationManager, a message can be reconstructed from any received byte stream during the simulation. The Message class also includes other attributes that are general for all kinds of messages: positive indicates if the message is a positive one or not; st is the time-stamp of the message; send_lp and recv_lps stores the identifier of the sending LP and receiving LPs (a multicast message may have multiple recipients) respectively; color is needed by Mattern’s GVT algorithm to carry out GVT approximation. In order to transfer messages in the form of byte 
stream across the network, serialize() and deserialize() of the MessageInterface class (see Figure 4.7) must be implemented in any derived message.

\subsubsection{State}

The State class (see Figure 4.8) is used as the base class of the state used in simulations. It is used to wrap all the state variables that are to be checkpointed during the course of a simulation. Besides the time-stamp, st, of the state, iq_pos/oq_pos remembers the position in the input/output queue up to which input/output messages have been processed/sent at time this state was reached. When an old state is recovered due to a rollback, its iq_pos and oq_pos are recovered as well. The knowledge of iq_pos and oq_pos is useful in implementing various cancellation schemes. Using aggressive cancellation scheme, for example, any input message after iq_pos is required to be unprocessed and any output message after oq_pos is required to be cancelled by sending its corresponding anti-message. alloc_state0 of the Statelnterface class (see Figure 4.8) needs to be implemented by application developers in State derived classes so that the executive is able to allocate storage for a user-defined state when manipulating states.

\begin{tabular}{|l|}
\hline \multicolumn{1}{|c|}{ State } \\
\hline SIMTIME st \\
InputQueue: : iterator iq_pos \\
OutputQueue: : iterator oq_pos \\
\hline
\end{tabular}

\begin{tabular}{|c|}
\hline StateInterface \\
\hline State* alloc_state ()$=0$ \\
\\
\hline
\end{tabular}

Figure 4.8: State Class and StateInterface Class

\subsubsection{Input Queue, Output Queue, State Queue}

The input queue, output queue and state queue used in an LP are represented as the InputQueue class, Outputqueue class and Statequeue class (see Figure 4.9) respectively. Similar to most Time Warp implementations, these classes employ a linear data structure 
and list operations to manage input messages, output messages and states. Note that different state saving strategy may have different requirements on garbage collection. Thus, the implementation of $\mathrm{g} \mathrm{c}_{\mathrm{C}}$ ) in each queue is left for executive developers.

\begin{tabular}{|c|c|}
\hline InputQueue & OutputQueue \\
\hline 1 ist< Message* > msgs & \begin{tabular}{c}
$\mid$ ist< Message* > msgs \\
\hline$/ *$ list operations $* /$
\end{tabular} \\
\hline
\end{tabular}

\begin{tabular}{|c|c|}
\hline StateQueue & QueueInterface \\
\hline list< State* > states & \\
\hline$/ *$ list operations $* /$ & void gc(SIMTIME st) $=0$ \\
\hline
\end{tabular}

Figure 4.9: InputQueue Class, OutputQueue Class, StateQueue Class and QueueInterface Class

\subsubsection{Communication Manager and GVT Manager}

Communication support and periodical GVT approximation are necessary for Time Warp simulation. Since they are general to most simulations, ATW has a full implementation of them in classes CommunicationManager and GVTManager respectively. The CommunicationManager class optionally interacts with the Network Emulation Module and send/receive messages to/from the underlying network using MPICH library. The GVTManager class does GVT approximation using Mattern’s algorithm [98]. It is expected that these two classes can be directly reused in any derived executives so that executive developers can concentrate on the implementation of the interfaces presented above.

\subsection{Generalized Mechanisms in Abstract Time Warp}

\subsubsection{Flow of a Simulation}

ATW can be easily extended and configured for new executives. Most common Time Warp mechanisms are generalized in ATW. Understanding how these mechanisms work in 
ATW is important for executive/application developers to carry out their implementation in derived executives and applications. As shown in Figure 4.10, any simulation using this extensible framework has a unique flow.

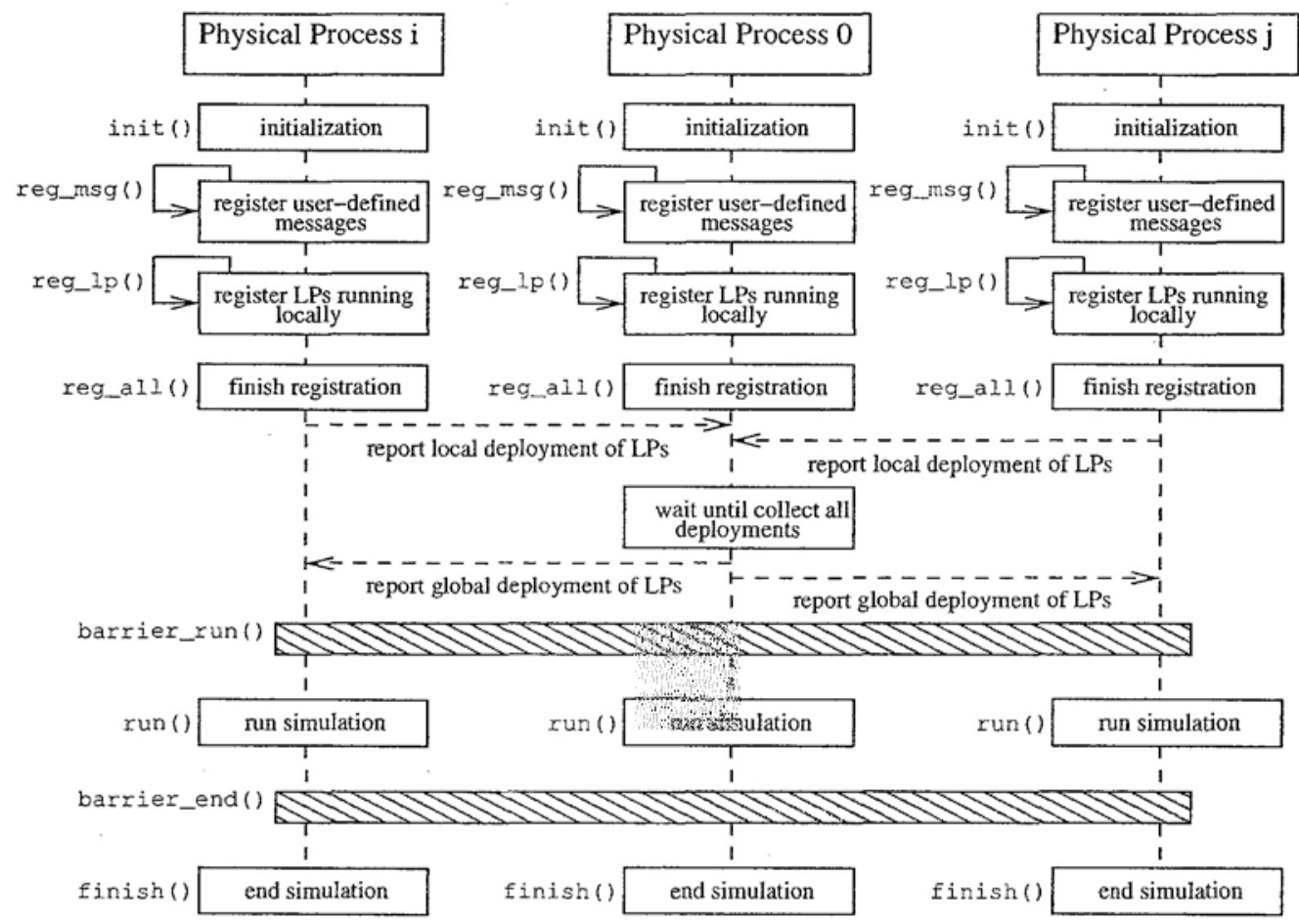

Figure 4.10: Flow chart of Time Warp simulation

The lifetime of a simulation is divided into three stages, which are delimited and coordinated at two different barriers (barrier_run() and barrier_end()) shown in the figure. In the first stage, a simulation starts with a number of physical processes running on an identical number of processors. Then initialization is carried out for the executive and the application by calling their init() function: (1) Initialization of the executive includes setting up the communication manager, the GVT manager and registering the messages used by the executive; and (2) Initialization of the application mainly sets up customized environment for the simulation. Application messages are registered with the executive by calling reg_msg(). Their allocators and serialization/deserialization functions are known by the communication manager so that any message sent to and 
received from the underlying network can be handled correctly. The deployment of LPs has two steps: (1)The view of local deployment is first generated at each physical process. This is done by calling reg_lp() with each LP's identifier that is going to be managed by the physical process. (2) Local deployment ends up with calling reg_all (). The physical process with identifier zero is then selected as the server to receive local deployments from other physical processes and then broadcast them with the generated view of global deployment. A global deployment is a list of pair of identifiers < ppid,lpid $>$ with identifiers of all the physical processes and LPs being included and mapped. Knowing the global deployment, each physical process is then able to send the messages to the right destination. Up to this point, all the LPs are ready and synchronized to run upon reaching the first barrier. The second stage is the major part of the simulation. Although this stage is more executive specific and involves running of application codes, thanks to the abstraction, it is still possible to be generalized using the mechanism discussed in Section 4.4.2. After all the LPs finishes, the simulation is to end. These LPs are synchronized again at the second barrier and then carry out their clean-up respectively.

\subsubsection{Running Physical Process}

The default implementation of PhysicalProcess: :run( ) is shown in Figure 4.11, which demonstrates a generalized control of Time Warp simulation running on each processor.

Right after reaching the first barrier, physical processes are now ready to run and communicate with each other throughout the simulation. Each physical process enters a loop, which loops until all the LPs have finished its simulation. At the beginning of each cycle, an LP is selected from the logical process pool by calling lpp->schedule(). In order to reduce LCC violations, only the LP having an unprocessed message with the smallest time-stamp is selected. Next, lp->run() is called, which basically carries out three tasks: (1) It checks if processing of the first unprocessed message would cause a 
LCC violation and, if so, rolls back the LP to the right previous state; (2) If no rollback is made, current state of the LP is checkpointed; and (3) The first unprocessed message is returned and subsequently passed into lp->process () for further processing. Note that because of the polymorphism, $1 \mathrm{p}->\operatorname{run}()$ and $l p->$ process () actually execute the code in the derived classes. This mechanism significantly unifies the logic in the framework while exposing great extensibility to executives and applications (Key routines that are used to implement $1 \mathrm{p}->$ run( ) and $1 \mathrm{p}->$ process () in BTW and CTW are presented in Section 5.5.2 and Section 6.4 respectively). As a part of the cycle, the communication manager and the GVT manager are also scheduled to run concurrently. The communication manager not only retrieves arrived messages from the underlying network, but also transmits all the messages sent during this cycle. The GVT manager runs without requiring any specific application knowledge. Relevant information such as the time-stamp, the color and the identifiers of the sending LP and the receiving LPs of every message going through the communication manager is collected so that the GVT approximation can proceed transparently. The last step of each cycle is garbage collection, which is carried out using the GVT just obtained.

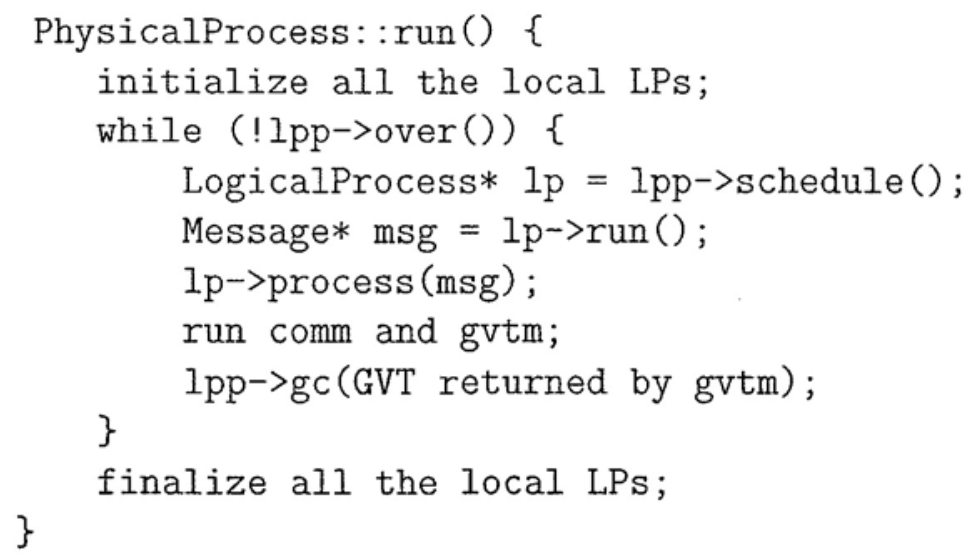

Figure 4.11: Default implementation of PhysicalProcess: :run () . 


\subsection{Summary}

In this chapter, an extensible framework for Time Warp simulation kernel (TWSK) is presented. The introduction of this framework is based on the general two-level approach in developing Time Warp simulations. Such a framework is required because the implementation of a full functional Time Warp executive is non-trivial and a unified interface to the application is desirable for various executives. Our research on Time Warp simulations requires major changes in traditional Time Warp executive and fair comparison between executives using the same benchmark application. This motivates the development of TWSK.

The design of TWSK follows an object-oriented approach and targets to two roles of developers. The Time Warp mechanism is abstracted into a minimum set of classes, namely, ATW, which is highly configurable. ATW is not a real executive, but it provides reusable and necessary facilities (e.g., GVT approximation and communication support) and defines the way, by providing interface, that a real executive should follow. Our implementation of three real executives, i.e., TTW, BTW and CTW, proves that ATW is useful in developing new executives and simulation applications developed using TWSK can run on top of any of these executives with minimal changes (refer to Appendix A for a real example).

Besides various Time Warp algorithms, e.g., state checkpoint algorithms, cancellation algorithms, that are configurable in TWSK, TWSK also supports sequential simulation. This is obtained by using only one physical process and register all the LPs with it. The way that the logical process pool schedules the LPs coincides with that of sequential simulation. Since messages are sent internally in the physical process, the communication manager just loops back any outgoing message. 


\section{Chapter 5}

\section{Batch Based Cancellation Scheme}

\subsection{Introduction}

In traditional Time Warp simulations, the conventional way of cancelling events is solely carried out on a per-event basis. This is carried out in a way that when an LP decides to cancel an external event which was previously scheduled by an outgoing positive message, it proceeds by constructing its counterpart, namely, an anti-message, and sending it to the same destinations. Upon receipt of the positive message and its anti-message, the receiving LP cancels the scheduled event and performs necessary rollbacks if that event has been processed.

The above cancellation scheme empirically proves to be simple and feasible in most Time Warp simulations. But, it still leaves performance concerns behind and has drawn researchers' attention. Given that LPs proceed aggressively in a Time Warp simulation, during the period between processing an incoming positive message and receiving its antimessage, a certain amount of events may have been scheduled by an LP. Without loss of generality, the computation of these events are dependent on the cancelled event, hence need to be rolled back by means of sending further anti-messages. For the cancellations triggered by a straggler message, it is apparent that the cost is mainly dominated by 
the way LPs process and cancel events. In the presence of dynamic CPU workload and varying communication delay in a parallel or a distributed simulation, the cost is hardly predictable.

Several pitfalls in Time Warp simulations which are results of costly cancellations have been investigated by researchers, which are illustrated as follows.

- Chasing Hazard [59]: It takes time to send an anti-message to its destination. If the spreading speed of incorrect computations is faster than that of anti-message, the whole simulation fails. This pitfall is shown in Figure 5.1 $1^{5.1}$. Before $L P_{2}$ receives anti-message $m_{1}^{-}$, it already scheduled an incorrect event by message $m_{2}$ (see Figure 5.1(a)). Upon receipt of $m_{1}^{-}, L P_{2}$ rollbacks and sends $m_{2}$ 's anti-message $m_{2}^{-}$to $L P_{3}$. However, $L P_{3}$ has already processed $m_{2}$ and scheduled other incorrect events (see Figure 5.1(b)).

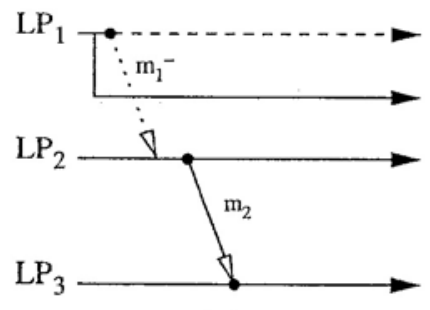

(a)

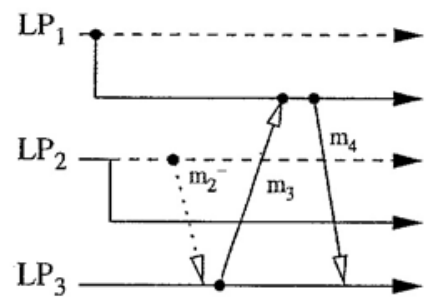

(b)

Figure 5.1: Anti-messages chase incorrect computations

- Rollback echoes [59]:If the time to perform a rollback is long and proportional to the number of events to be rolled back, a pitfall called rollback echoes may happen. As shown in Figure 5.2, assume that rollback of $n$ events takes the same time as processing $2 \times n$ events and time-stamp increment is identical for scheduling any event. Initially, $L P_{2}$ receives straggler message $m_{1}$ from $L P_{1}$ and rolls back two

\footnotetext{
${ }^{5.1}$ To better present the rollback behavior in LPs' advancement, tree diagram is used in Chapter 5 and Chapter 6, in which a new branch ramifies at the moment the LP recovers from a rollback. For more detailed description of the tree diagram, please refer to Section 6.3.
} 
events. Because rollback takes more time, $L P_{1}$ may process four events in the meantime. This increases the likelihood of receiving straggler message, e.g., $m_{2}$, from $L P_{2}$. With our assumption on time-stamp increment, $m_{2}$ probably causes $L P_{1}$ to rollback four events, during which $L P_{2}$ could process yet another eight events. If $L P_{1}$ causes $L P_{2}$ to rollback again by message $m_{3}$, it is likely that eight events have to be rolled back at $L P_{2}$. This echo behavior demonstrates that increasing the cost of rollback could result the computation totally fruitless.

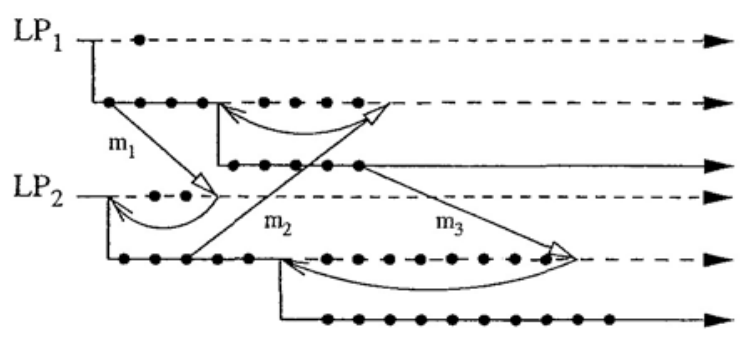

Figure 5.2: Rollback echoes

- Cascading rollbacks [29]: Because the propagation of anti-messages and the spreading of computations are in parallel, the LP which initiated a rollback, either due to receiving a straggler message or an anti-message, may again enter a state that is dependant on an event that it has just rolled back. This is shown in Figure 5.3. While $L P_{1}$ is sending the anti-message $m_{1}^{-}$to $L P_{2}$, message $m_{2}$, which is scheduled by processing $m_{1}$, is on its way to $L P_{1}$. Because $L P$, has no idea that $m_{2}$ is related to $m_{1}$, processing of $m_{2}$ will make $L P_{1}$ suffer from another rollback.

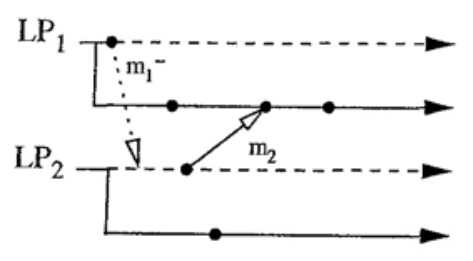

Figure 5.3: Cascading rollbacks 
- Unnecessary multiple rollbacks [29]: Because per-event based cancellation does not concern the relationship of anti-messages that generated in a course of rollback triggered by a straggler message, unnecessary multiple rollbacks may occur at a certain LP. As shown in Figure 5.4, the receipt of a straggler message at $L P_{1}$ results in anti-message $m_{1}^{-}$and $m_{2}^{-}$being sent from $L P_{1}$ to $L P_{2}$ and $L P_{3}$, and $m_{3}^{-}$ being sent from $L P_{2}$ to $L P_{3}$ respectively. Assume that $S T\left(m_{2}\right)<S T\left(m_{3}\right)$ and $m_{2}^{-}$is received before $m_{3}^{-}$at $L P_{3} . L P_{3}$ may suffer from an unnecessary additional rollback caused by $m_{3}^{-}$, since $m_{3}$ may be processed again after a rollback caused by $m_{2}^{-}$.

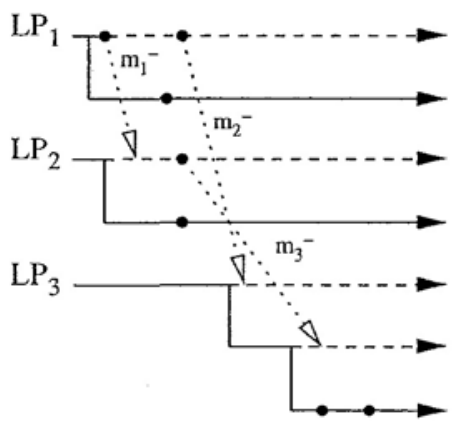

Figure 5.4: Unnecessary multiple rollbacks

\subsection{Motivations}

To address the above mentioned pitfalls, there are many approaches that have been proposed to reduce the cost of cancellations. As opposed to aggressive cancellation, lazy cancellation [60] reduces the number of anti-messages by delaying the sending of anti-messages till reprocessing of the event produces a different positive message. Unfortunately, the performance comparison shows that lazy cancellation can arbitrarily outperform aggressive cancellation and vice versa [120]. Lazy re-evaluation [146] lets an LP directly jump back to the state prior to when the rollback occurred if the straggler message does not affect its state. To avoid chasing hazards, wolf call [93] expedites the 
propagation of the knowledge of cancellations by sending protocol messages with higherpriority over positive messages in their transmission. However, all these approaches do not address the inefficiency of the conventional per-event based cancellation scheme itself.

There are some other cancellation schemes that aim to provide faster and more powerful cancellation by using additional information carried on messages and possible replacement of traditional anti-message that is capable of cancelling multiple messages. They are discussed as below.

- Filter [111]:The mechanism of Filter is essentially based on the following observation: let tuple $<c$, seq, st $>$ denote that no straggler message will be received on channel c with a sequence number greater than seq, and time-stamp smaller than $s t$. For any two tuples $\left\langle c, s e q_{a}, s t_{a}\right\rangle$ and $\left\langle c, s e q_{b}, s t_{b}\right\rangle$, they have a conflict iff $s e q_{i a}<s e q_{i b}$ but $s t_{i a}>s t_{i b}$, which means the first state must have been discarded before the second state is reached. In the filter approach, rollback list and assumption list are constructed as lists of such tuples. Assumption list is maintained by LPs and piggybacked by messages, which represents an accumulated knowledge of tuples from all the channels. Rollback list is only maintained by LPs, which records the tuples identifying all the straggler messages known so far. By comparing the tuples in an LP's rollback list and the assumption list in a received message, the message can be discarded preemptively if a conflict is found. Note that Filter still uses anti-message.

- Dependence List Cancellation [151]: Dependence lists is a tree structure that is built upon the events internal to the same processor on which multiple LPs are running. Each edge in the tree represents a scheduling dependence [59] either internal to the processor or traversing across other processors. For the purpose of capturing the latter, each event generated in the simulation carries a vector, in 
which each entry records the event in the corresponding processor that current event depends on. Apparently, dependence lists can assist fast cancellation of dependent events internal to the processor once an erroneous event is detected. To do so, a traversal is made from the erroneous event down to the leaves of the tree. All the events along the path are to be cancelled. The use of Dependence lists may also stop cascading rollbacks. Upon receipt of an event coming from a different processor, its internally dependent event is obtained from its carried vector. If the latter has already been marked as being cancelled, the former is discarded right away. In order to prevent cascading rollbacks from happening, all the events, being processed and cancelled, in the processor are required to be kept in the dependence lists until fossil collection. Cancellation of events external to the processor still uses anti-message.

- Transitive Dependency Tracking [35]:The number of rollbacks, namely, incarnation number, at each LP is checkpointed as a part of the state. The relationship of messages is captured using a variant of vector time, namely Simulation Vector, in which each entry is extended to a tuple $<i n c$, st $>$. The $i^{t h}$ entry $<i n c$, st $>$ of a simulation vector means that a rollback at $L P_{i}$ restored its incarnation number back to inc at simulation time st. The Simulation Vector locally maintained at each LP is sent with outgoing messages and updated when receiving any message. Thus the dependency on the latest state of any LP is known. Suppose that $L P_{i}$ receives a straggler message, the cancellation is carried out in a block-and-wait manner. The knowledge of $<$ inc, st $>$ of the state recovered at $L P_{i}$ is first broadcast to all the other LPs. Upon receipt of this tuple, the receiving LP identifies and rolls back all dependent states/messages and then replies $L P_{i}$ an acknowledgement. The dependency is evaluated by comparing receiving LP's state/message's $i^{\text {th }}$ entry $<$ inc', st' $>$ with $<i n c$, st $>$. If inc' $>i n c$ or inc' $=i n c \wedge s t^{\prime}>s t$ holds, then 
the state/message is known dependent on the state just having been rolled back at $L P_{i}$. After $L P_{i}$ receives the replies from all the other LPs, it then resumes.

- Range based Cancellation [29]: This approach uses vector time to capture the relation between messages. The combination of simulation time and vector time forms the Total Clock used in the simulation. The updating rules of simulation time and vector time are independent from each other and correspond to those defined in $[29,71,80]$. The cancellation mechanism is shown in Figure 5.5, in which the dashed arrows represent the protocol messages, namely, CANCEL_MESSAGES, that are used to cancel multiple messages. Each CANCEL_MESSAGE carries a range, denoted by $[a, b]_{i}$, which indicates that the set of events that have been cancelled at $L P_{i}$ are those whose $i^{\text {th }}$ component of vector time lies in between $a$ and $b$. Based on a received range, the receiving $L P$ cancels events by checking their vector time. A new range for the cancelled events is then deduced and sent out via new CANCEL_MESSAGES (if the range is not empty).

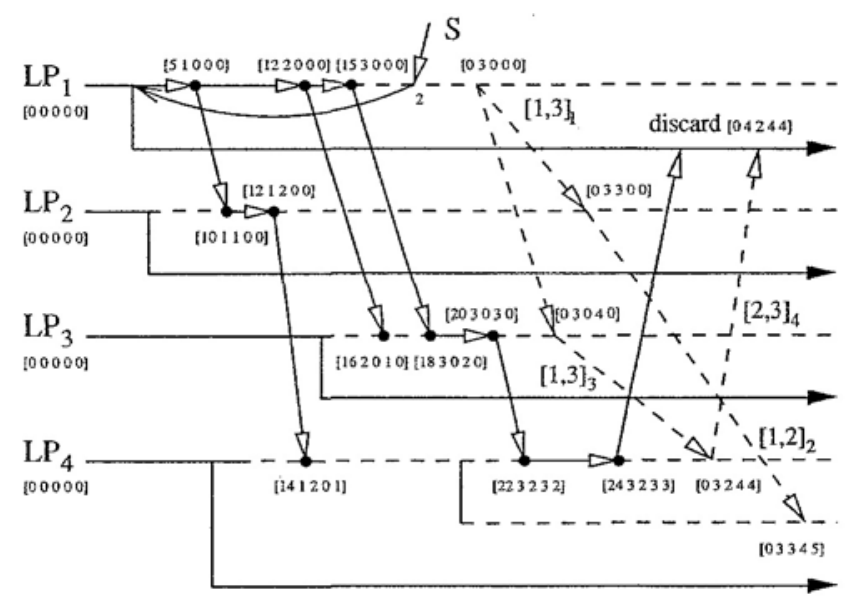

Figure 5.5: Range based cancellations triggered by a straggler message

All these cancellation schemes can reduce cancellation cost and help avoid above mentioned pitfalls. However, they have different abilities to prevent multiple rollbacks 
from happening. We define the term rollback optimal as follows.

Definition 5.2.1 (Rollback Optimal). Regarding the number of rollbacks from which an LP may suffer to thoroughly remove the wrong computations incurred by a certain straggler message, a cancellation scheme is said to be rollback optimal if any affected LP is guaranteed to be recovered at the cost of at most one rollback.

It can be seen that neither filter nor dependence list cancellation nor range based cancellation is rollback optimal. During the course of rollbacks caused by receiving a straggler message, they can only determine incomplete set of events to be eventually cancelled during each rollback. For example for range based cancellation in Figure 5.5, $L P_{4}$ rolls back twice because $\mathrm{e}_{4,14}{ }^{5.2}$ is not able to be rolled back by the first rollback triggered by range $[1,3]_{3}$. Transitive dependency tracking is rollback optimal, however, it requires broadcast of messages and block-and-wait control, which potentially reduces parallelism. Analysis of these mechanisms motivates us to seek a more general rollback optimal cancellation mechanism.

\subsection{State Vector}

In Time Warp simulations, the basic rule is that the cancellation is carried out based on dependence. The dependence of events can be expressed using state dependence or scheduling dependence or a transitive closure of both [59]. For any two processed but not yet rolled back events at the same LP, the later event is defined to be state dependent on the earlier event due to the order of their accesses to the LP's state variables. For an event scheduling another event, the latter is defined to be scheduling dependent on the former. To remove the wrong computations based on an event when it is rolled back, all

\footnotetext{
${ }^{5.2} e_{i, t}$ is different from the notation of event enumeration in Definition 2.1.2. To simplify the represen-
} tation, $e_{i, t}$ denotes the event scheduled at $L P_{i}$ with time-stamp $t$. 
events that depend on it have to be cancelled ${ }^{5.3}$.

The causal order proves to be the most basic but universal partial order relation in distributed systems and vector time proves to be the simplest means to characterize it. However, vector time (see Definition 2.1.8) is not an ideal candidate to capture dependence of events. This is illustrated in Figure 5.6. $L P_{i}$ schedules $e_{j, t_{2}}$ and later on cancels it due to the rollback caused by a straggler message. For events $e^{\prime}$ and $e^{\prime \prime}$ scheduled by $L P_{j}$, where e' is scheduled after $e_{j, t_{2}}$ but before the cancellation and $e^{\prime \prime}$ is scheduled after the cancellation, both $e_{i, t_{1}} \rightarrow e^{\prime}$ and $e_{i, t_{1}} \rightarrow e^{\prime \prime}$ hold. But, in fact, $e^{\prime \prime}$ does not depend on $e_{i, t_{1}}$.

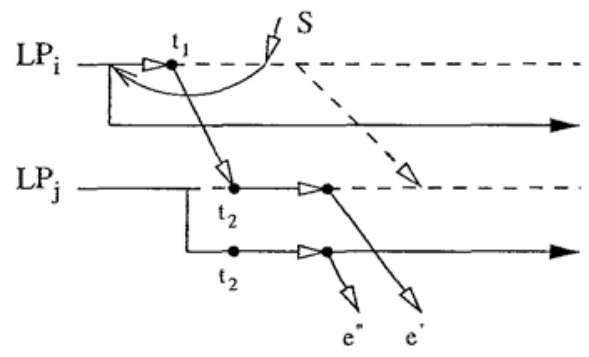

Figure 5.6: Happen-before relation between events

In order to correctly capture the dependency between events, we propose a variant of vector time, namely, state vector (SV). Suppose that every LP changes its state whenever it processes an event. A monotonically increasing scalar, namely, state counter, is introduced into each LP to uniquely identify its state. A state vector is a vector of state counters which is maintained at each LP. For $L P_{i}$ 's state vector, denoted as $S V\left(L P_{i}\right)$, $S V\left(L P_{i}\right)[j], j \neq i$, reflects $L P_{i}$ 's current knowledge of $L P_{j}$ 's latest state counter, while $S V\left(L P_{i}\right)[i]$ is just the placeholder of its own state counter.

The propagating and updating rules of $L P_{i}$ 's state vector are shown in Figure 5.7. It can be seen that the last rule makes state vector different from vector time.

\footnotetext{
${ }^{5.3}$ Note that “cancel” means not only a rollback of a processed event but also a cancellation of an unprocessed event.
} 
1. Let $N$ be the number of LPs in the simulation. Initially, $S V\left(L P_{i}\right)[k]=0,0 \leq i, k<N$.

2. Let $S V_{m}(e)$ be the state vector of $e$ which is piggybacked on its scheduling message $m$ and let $S V(e)$ be the state vector of e at the time $e$ is processed. When $L P_{i}$ processes $e$, these updates are performed: $S V\left(L P_{i}\right)[i]$ first increases by one; then $S V\left(L P_{i}\right)=$ supreme $\left(S V\left(L P_{i}\right), S V_{m}(e)\right)$, where supreme performs componentwise maximum operation; then $S V(e)=S V\left(L P_{i}\right)$.

3. At the time $L P_{i}$ schedules event e, either for itself or for another LP, $S V\left(L P_{i}\right)$ is piggybacked on the scheduling message, i.e., $S V_{m}(e)=$ $S V\left(L P_{i}\right)$.

4. Except for $S V\left(L P_{i}\right)[i]$, other components of $S V$ are state saved. At the time a rollback occurs at $\mathrm{LPi} S V\left(L P_{i}\right)\left[_{i}\right]$ increases by one and other components are restored from the state to which $L P_{i}$ rolls back.

Figure 5.7: Propagating and updating rules of state vector at $L P_{i}$

With the rules in Figure 5.7, $S V\left(L P_{i}\right)[i]$ uniquely identifies an event processed at $L P_{i}$. Given an event e processed by an LP with state vector $S V(e)$, the latest event in $L P_{i}$ on which $e$ depends can, therefore, be determined by $S V(\mathrm{e})[\mathrm{i}]$ (formally stated in

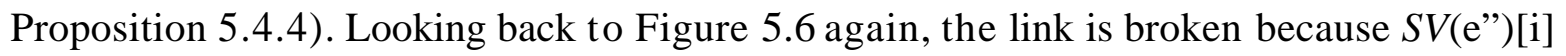
is restored from an earlier state. $S V\left(e^{\prime \prime}\right)[i]<S V\left(e_{i, t_{1}}\right)[i]$ indicates that e" does not depend on $e_{i, t_{1}}$. Other properties of state vector and dependence are exploited in Section 5.4.

\subsection{Characterization of Cancelled Events}

The widely accepted model (see Definition 2.3.3) is used in the proof of the following theorems. A Time Warp simulation is viewed as consisting of $\mathrm{N}$ sequential LPs, communicating solely by exchanging two kinds of basic messages, namely, positive messages and CANCEL_MESSAGES. Each LP changes its state whenever it processes the event 
scheduled by a positive message or performs a rollback. The transmission channels are assumed reliable and FIFO.

Recall Definition 2.3.2, events in Time Warp simulation can be defined similarly as below. Let $E_{i}$ denote the set of events processed at $L P_{i}$, and let $E=\bigcup_{i=1}^{N} E_{i}$ denote the set of all events processed in the simulation. As we assume that each $L P_{i}$ is strictly sequential, we can index the events of $L P_{i}$ in their processing order: $E_{i}=\left\{e_{i 1}, e_{i 2}, \ldots, e_{i n}, \ldots\right\}$. This processing order is referred to as the standard enumeration of Ei. Given Ei, the local successor set of event $e_{i n}$, denoted as $\mathcal{S}_{l}\left(e_{i n}\right)$, represents all the events locally processed after $e_{i n}, \mathcal{S}_{l}\left(e_{i n}\right)=\left\{e_{i k} \mid k>n\right\}$.

Proposition 5.4.1 through Proposition 5.4.5 state the properties of state vector and the dependence of events. They are obvious by applying the rules shown in Figure 5.7 and are listed below without proving.

Proposition 5.4.1. Let $e \Rightarrow e$ ' denote that event $e$ depends on $e$. Dependence is transitive, i.e., for three events $e$, $e^{\star}$ and $e^{»,}$ if $e \Rightarrow e^{\prime}$ and $e^{\prime} \Rightarrow e^{»}$, it also holds that $e \Rightarrow e^{\prime \prime}$

Proposition 5.4.2. $S V\left(L P_{i}\right)[i]$ increases monotonically throughout a simulation.

Proposition 5.4.3. For any event $e_{i k} \in E_{i}, S V\left(e_{i k}\right)[i]=k$.

Proposition 5.4.4. For event $e^{\prime} \in E_{j}$, suppose that $S V\left(e^{\prime}\right)[i]=k$, then $e_{i k} \Rightarrow e^{\prime}$.

Proposition 5.4.5. For events $e_{i k} \in E_{i}$ and $e^{\prime} \in E_{j}$, if $e_{i k} \Rightarrow e^{\prime}$, then $S V\left(e^{\prime}\right)[i]>=k$.

Observing that receiving straggler messages is the root cause of rollbacks in LPs, let $m$, denote a straggler message received at $L P_{\mathrm{O}} \cdot L P_{\mathrm{o}}$ is thus named as rollback originator. During the cancellations triggered by $m_{\mathrm{s}}$ (for the ease of discussion, assume there is no other intervening cancellations triggered by other straggler messages), let $C_{\mathrm{i}}\left(m_{\mathrm{s}}\right)$ denote the set of events cancelled at $L P_{\mathrm{i}}$ and $C\left(m_{s}\right)$ denote all the events cancelled in the 
simulation. Thus, $C\left(m_{s}\right)=\bigcup_{i=1}^{N} C_{i}\left(m_{s}\right)$. Note that for $i \neq o, C_{i}\left(m_{s}\right)$ is essentially the set of events that have dependence on the events in $C_{o}\left(m_{s}\right)$, i.e., $\left\{e^{\prime} \mid e^{\prime} \in E_{i} \wedge \exists e \in\right.$ $\left.C_{o}\left(m_{s}\right), e \Rightarrow e^{\prime}\right\}$.

Although $C\left(m_{s}\right)$ can be expressed in terms of $C_{o}\left(m_{s}\right), C_{o}\left(m_{s}\right)$ or $C\left(m_{s}\right)$ is still unpredictable without any regulation of the advancement of LPs. To be able to identify $C_{o}\left(m_{s}\right)$, which is expressed as arange (shown in Theorem 5.4.2), and furthermore, identify $C\left(m_{s}\right)$ using this range (shown in Theorem 5.4.3), the rules of event processing at $L P_{i}$ are given in Figure 5.8. In the first rule, if event e is processed, it would be rolled back unnecessarily because $e$ ' will be eventually cancelled. In the second and the third rules, event $e$ can be discarded immediately as it is known to be dependent on a cancelled event.

At the time $L P_{i}$ processes event e, where $S V_{m}(e)=\left\{x_{1}, x_{2}, \ldots, x_{N}\right\}$,

1. If a processed (but not yet committed) event $e^{\prime}$ at $L P_{i}$ depends on a cancelled event $e_{j k}, j \neq i, k \leq x_{j}$ on which $e$ does not depend, processing of $e$ is blocked until $\dot{e}$ has been cancelled;

2. If $e_{i x_{i}}$ has been cancelled locally, $e$ is discarded without processing;

3. If e depends on a cancelled event $e_{j k}, j \neq i, k \leq S V\left(L P_{i}\right)[j]$, e is discarded without processing.

Figure 5.8: The rules of event processing at $L P_{i}$

Theorem 5.4.1. Suppose that the rollback originator $L P_{o}$ receives straggler message $m_{s}$ and events $e_{o a}$ and $e_{o b}$ are the earliest and latest events among those being cancelled by LP. It holds that,

$$
C_{o}\left(m_{s}\right)=\left\{e_{o a}\right\} \cup\left(\mathcal{S}_{l}\left(e_{o a}\right)-\mathcal{S}_{l}\left(e_{o b}\right)\right) .
$$

PROOF: 
The event set $\left\{e_{o a}\right\} \cup\left(\mathcal{S}_{l}\left(e_{o a}\right)-\mathcal{S}_{l}\left(e_{o b}\right)\right)$ represents all the events cancelled by $L P_{o}$ upon receipt of straggler message $m_{s}$ The second rule in Figure 5.8 prohibits their effects from further spreading at $L P_{o}$, hence this set essentially equals $C_{o}\left(m_{s}\right)$.

Theorem 5.4.2. The $o^{\text {th }}$ component of the state vector of events in $C_{o}\left(m_{s}\right)$ forms a continuous range $[a, b]_{o}$, that is,

1. $\forall k \in[a, b]_{o}, e_{o k} \in C_{o}\left(m_{s}\right)$;

2. $\forall e_{o k} \in E_{o}$ and $e_{o k} \notin C_{o}\left(m_{s}\right), k \notin[a, b]_{o}$.

\section{PROOF:}

From Proposition 5.4.3, $S V\left(e_{o a}\right)[0]=a$ and $S V\left(e_{o b}\right)[o]=b$. According to Proposition 5.4.2 and Theorem 5.4.1, the correctness is obvious.

Theorem 5.4.2 offers an efficient way to convey the set of events being cancelled at a rollback originator to other LPs (recall the similar way in which CANCEL_MESSAGES work in the range based cancellation scheme). As shown in the next section, our proposed scheme also employs CANCEL_MESSAGES to carry these ranges.

Theorem 5.4.3. Given range $[a, b]_{o}$ representing $C_{o}\left(m_{s}\right), \forall e \in E_{i}, i \neq o$, $e \in C_{i}\left(m_{s}\right) \quad$ iff $\quad S V(e)[o] \in[a, b]_{o}$.

\section{PROOF:}

(1)Necessity. On one hand, for any event $e \in \operatorname{Ci}\left(m\right.$, ), it holds that $\exists k \in[a, b]_{o}, e_{o k} \Rightarrow$ e. Applying Proposition 5.4.5, $S V(e)[o] \geq k \geq a$. On the other hand, it must hold that $S V(e)[o] \leq b$. Otherwise, assume $S V(e)[o]=k^{\prime}>b$, then $e_{o k^{\prime}} \Rightarrow e$ according to Proposition 5.4.4. $e_{o k^{\prime}}$ does not depend on any event in $C_{o}\left(m_{s}\right)$, or it should have been discarded according to the second rule in Figure 5.8. Therefore, e must have dependence on other events as shown in Figure 5.9, where dependence of events is denoted as curved 
arrows. There must be two events $e^{\prime \prime}$ and $e^{\prime}$ which were processed in this order at $L P_{i}$, $i \neq o$ : e depends on $e^{\prime}$ and $e^{\prime \prime}$ depends on an event $e_{o k^{\prime \prime}}$ in $C_{o}\left(m_{s}\right)$ as $e \in C_{i}\left(m_{s}\right)$; is identical to or depends on $e^{\prime}$ and $e^{\prime}$ depends on $e_{o k^{\prime}}$ as the knowledge of $k$ was propagated to $e ; e^{\prime \prime}$ was processed before $e$ ' (otherwise, $e$ " was discarded by $L P_{i}$ according to the third rule in Figure 5.8). This violates the first rule in Figure 5.8 at the time $L P_{i}$ processed $e^{\prime}$ because processing of $e$ should have been blocked until $e^{\prime \prime}$ was cancelled. (2) Sufficiency. Let $S V(e)[o]=k, k \in[a, b]_{o}$. Applying Proposition 5.4.4, $e_{o} k \Rightarrow$ e. Because $e_{o k} \in C_{o}\left(m_{s}\right)$, $e \in C_{i}\left(m_{s}\right)$.

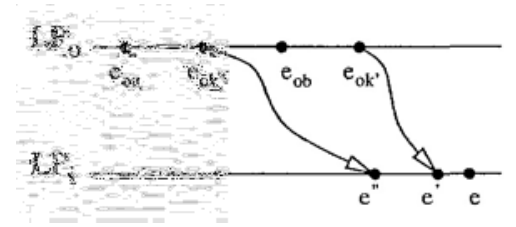

Figure 5.9: The dependence of event e

The rules in Figure 5.8 and Theorem 5.4.1 through Theorem 5.4.3 form the basis for a rollback optimal cancellation scheme. The essential difference from the range based scheme is that instead of continuously constructing ranges along the propagation path of CANCEL-MESSAGES, which could gradually limit their abilities to determine events in $C_{i}\left(m_{s}\right), i \neq o$ (see ranges $[1,3]_{1}$ and $[1,3]_{3}$ in Figure 5.5), a rollback optimal cancellation scheme comes to the conclusion that $L P_{i}$ is able to directly deduce what $C_{i}\left(m_{s}\right)$ is like based on the received $[a, b]_{o}$ from the rollback originator $L P_{o}$. For any processed events in $C_{i}\left(m_{s}\right), L P_{i}$ performs a single rollback to cancel all of them; For any received but not yet processed events or any events arriving in future, $L P_{i}$ simply discards them if they are identified to be in $C_{i}\left(m_{s}\right)$. This ensures that any $L P$ will discard all those events to be cancelled by a straggler message in a batch manner and at the cost of at most one rollback (rollback optimal). 


\subsection{Batch Based Cancellation Scheme}

\subsubsection{Rollback History}

The rules in Figure 5.8 are essential for the correctness of the scheme. Empirically, these rules can be fulfilled by employing the concept of rollback history. We have shown in Theorem 5.4.2 and Theorem 5.4.3 that range $[a, b]_{o}$ identifies all the events in $C\left(m_{s}\right)$. The rollback history of an LP, denoted as $R H(L P)$, is actually defined as a list of those ranges that were locally generated or learnt from other LPs through message passing.

Piggybacking its current rollback history on any event scheduled by an LP makes it possible for other LPs to process the event in the way that satisfies the first rule in Figure 5.8. Particularly, at the time an LP processes event $e$, it first looks into those events that have been processed but not yet committed. If an event that is to be cancelled by a certain range in the rollback history carried by e is found, processing of e is delayed until that event is cancelled.

The greatest challenge to the above approach is the increasing size of rollback histories carried by events, which could incur significant communication cost and storage cost, and render the approach itself empirically useless. Thanks to the FIFO property of communication channels, communication cost can be reduced by sending rollback histories incrementally and reconstructing them at the receiving LPs. To do this, a similar technique to that of compressing vector time described in [127] is employed together with more sophisticated data structures.

$L P_{i}$ locally maintains a rollback history table, denoted as $\operatorname{RHT}\left(L P_{i}\right)$. For $1 \leq j \leq$ $N, j \neq i, R H T\left(L P_{i}\right)[j]$ records $L P_{i}$ 's latest knowledge of $R H\left(L P_{j}\right) . R H T\left(L P_{i}\right)[i]$ is the placeholder of $L P_{i}$, but in addition, each range inside is tagged with a scalar, and denoted as $[a, b]_{o}^{c}$, where $c$ was the current value of $L P_{i}$ 's state counter when the range was recorded. $L P_{i}$ keeps a last scheduling vector, denoted as $L S\left(L p_{i}\right)$, as well. $L S\left(L P_{i}\right)[j]$, 
$1 \leq j \leq N, j \neq i$, tracks the latest value of $L P_{i}$ 's state counter when an event was scheduled at $L P_{j}$.

Let $\Delta R H(e)$ denote the increment of the sender LP's rollback history piggybacked on e, and let $R H(e)$ denote its reconstructed rollback history at the receiving LP. The updating rules of $R H T\left(L P_{i}\right)$ are shown in Figure 5.10.

1. Initially, $\operatorname{RHT}\left(L P_{i}\right)[j], 0 \leq j<N$, is set empty, and $L S\left(L P_{i}\right)[j]=$ $0,0 \leq j<N$.

2. At the time $L P_{i}$ receives event $e$ from $L P_{j}, \Delta R H(e)$ is appended to the end of $R H T\left(L P_{i}\right)[j]$ and then emptied. The reconstructed $R H(e)$ is the current value of $R H T\left(L P_{i}\right)[j]$,represented by a pointer to the current end of the latter.

3. At the time $L P i$ processes an event $e$ from $L P_{j}, \forall[a, b]_{o} \in$ $R H(e),[a, b]_{o}^{S V\left(L P_{i}\right)[i]}$ is merged (without any duplication) into $R H T\left(L P_{i}\right)[i]$, and then $[a, b]_{o}$ is removed from $R H T\left(L P_{i}\right)[j]$.

4. At the time $L P_{i}$ schedules an event e to $L P_{i}, \Delta R H(e)$ is first set to the list of ranges in $\left\{[a, b]_{o} \mid[a, b]_{o}^{c} \in R H T\left(L P_{i}\right)[i] \wedge c>L S\left(L P_{i}\right)[j]\right\}$. $L S\left(L P_{i}\right)[j]$ is then updated with the current $S V\left(L P_{i}\right)[i]$.

Figure 5.10: Updating rollback history table in FIFO environments

Note that in the third rule, removal of ranges from $R H T\left(L P_{i}\right)[j]$ means that it is not an exact reconstructed $R H(e)$ in the strict sense. However, its correctness is obvious.

The rollback history table is also subject to fossil collection. After a GVT calculation cycle, let $x_{j}, 1 \leq j \leq N$, be the maximum state counter fossil collected at $L P_{j}$. Because it holds that there are no events (including transient events) dependent on any cancelled event $e_{j k}, k<x_{j}, \forall[a, b]_{j} \in R H T\left(L P_{i}\right),[a, b]_{j}$ is discarded if $b \leq x_{j}$. 


\subsubsection{Implementation}

We implemented a Time Warp simulation executive using the batch based cancellation scheme, namely, Batch Time Warp (BTW), based on the framework presented in Chapter 4. BTW extends ATW by implementing necessary interfaces. For the seek of clarity, we present at a higher level by selectively elaborating important functional routines instead of presenting individual interfaces. Note that these routines are not assumed one-to-one mapped to the interfaces: implementation of them may involve more than one interfaces.

An LP in BTW routinely maintains two queues, the input queue and the state queue. Because CANCEL_MESSAGES are processed at the time they are received and will not be rolled back for reprocessing, an input queue only queues positive messages. Let $E_{I Q_{i}}$ denote the currently buffered processed events, i.e., neither having been rolled back nor fossil collected, in $L P_{i}$ 's input queue $\left(I Q_{i}\right)$. It is obvious that $E_{I Q_{i}} \subseteq E_{i}$ and empirically only events in $E_{I Q_{i}} \cap C_{i}\left(m_{s}\right)$ are considered to be rolled back by the straggler message $m_{s}$ In order to keep track of the destination LPs of the messages sent in a particular state, a state variable Dest $_{i}$, which is the union of the destinations of all messages sent in this state, is introduced for $L P_{i}$. Once $L P_{i}$ is rolled back, the union of $D e s t_{i}$ stored in rolled back states captures the effected LPs to be informed of the CANCEL_MESSAGE. For this reason, outgoing messages are no longer recorded and the output queue is eliminated from BTW.

Let $\operatorname{Dest}(\mathrm{m})$ denote the set of destination LPs of message $m$. The major routines of the batch based cancellation scheme are presented in Figure 5.11 through Figure 5.14.

Figure 5.11 shows the steps for $L P_{i}$ to process a positive message from its input queue. $L P_{i}$ first checks if it is safe to proceed against Rule 1 in Figure 5.8. If it is safe to do so, $L P_{i}$ first pushes its current state to the back of its state queue (assume that frequent state 


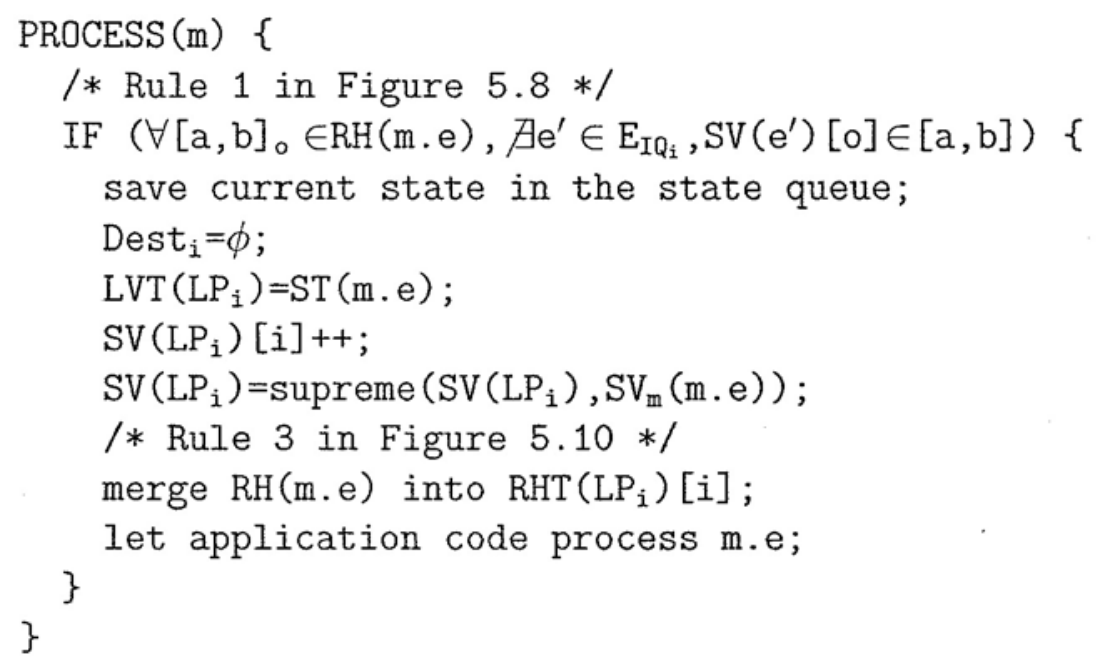

Figure 5.11: Processing a scheduled event at $L P_{i}$

saving is applied). $L P_{i}$ then continues with updating its simulation time, state vector and rollback history table, which are supposed to be internal to the simulation executive. The message is finally handed to the application code for processing, during which new events may be scheduled (Figure 5.12). Since possible receipt of straggler messages has been detected and handled at an earlier stage (see Figure 5.13), the messages to be processed here are already chronologically ordered without further checking for causality violations.

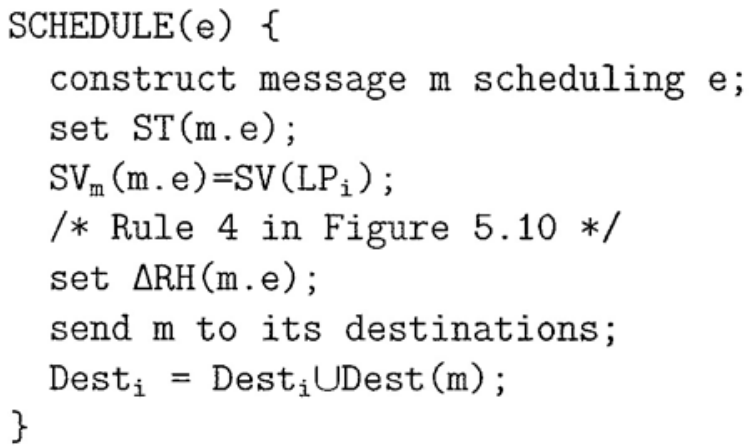

Figure 5.12: Scheduling an event at $L P_{i}$

Figure 5.12 shows necessary steps to be carried out in order to schedule an event. The construction of the message and setting its simulation time and destinations are initiated from the application code. Then, the simulation executive piggybacks required protocol 
information, i.e., current state vector of $L P_{i}$ and the increment of rollback history. For a message having multiple destination LPs, since the increment of rollback history is different for different LPs, the message is actually sent multiple times through pointto-point communication (an emulated multicast of the message): each message carries identical set of destinations, however, the increment of rollback history is calculated separately for its receiving LP. The last step is to record the destination of the message in the state variable Dest $_{i}$. If the current state is rolled back, a CANCEL_MESSAGE will be sent to these destinations.

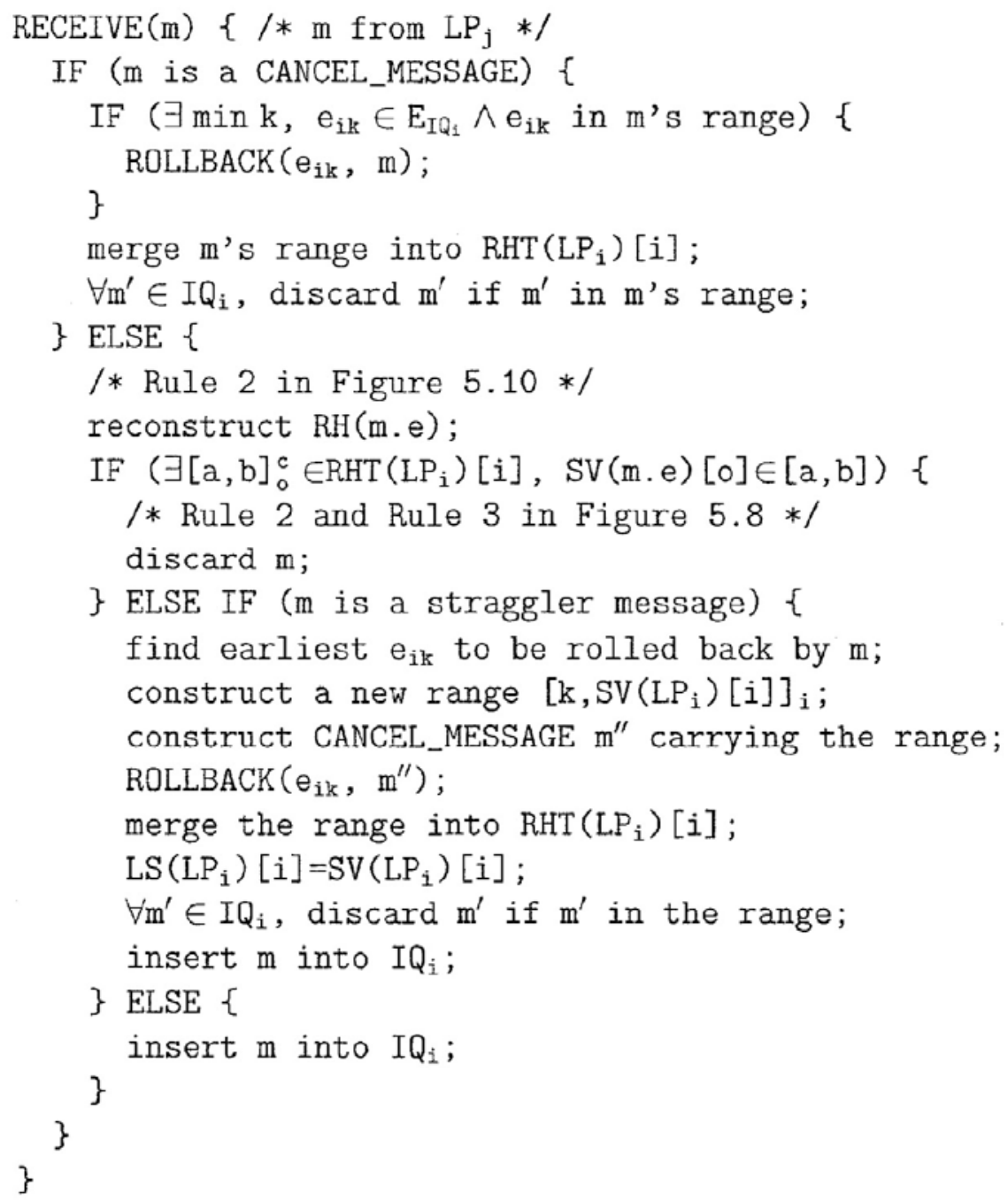

Figure 5.13: Receiving a message from communication channels at $L P_{i}$ 
Figure 5.13 demonstrates the handling of a message when it is received from the communication channel. The handling is internal to the simulation executive and transparent to the application code. Basically there are four different ways according to the criteria the message meets. (1) Message $m$ is a CANCEL_MESSAGE carrying a range. $L P_{i}$ first checks if any processed event falls within the range. If there is, $L P_{i}$ performs a rollback action (see Figure 5.14). Then $L P_{i}$ merges this range into its rollback history to identify any events for cancellation arriving in the future. Finally, $L P_{i}$ looks into its input queue to discard those events that have been received but are now identified to be cancelled. (2) Message $m$ is a positive message but identified as to be cancelled by a received range. It means that $L P_{i}$ is already free from the rolled back state on which $m$ depends. Thus, $m$ is simply discarded. (3) Message $m$ is a straggler message, so $L P_{i}$ becomes a rollback originator. Except for the need to create a new CANCEL_MESSAGE, similar actions to those in (1) are carried out. Finally, message $m$ is inserted into $L P_{i}$ 's input queue and expected to be the next message to be processed. (4) Message $m$ is a positive message identified as neither to be discarded nor a straggler. It is simply inserted into $L P_{i}$ 's input queue for future processing.

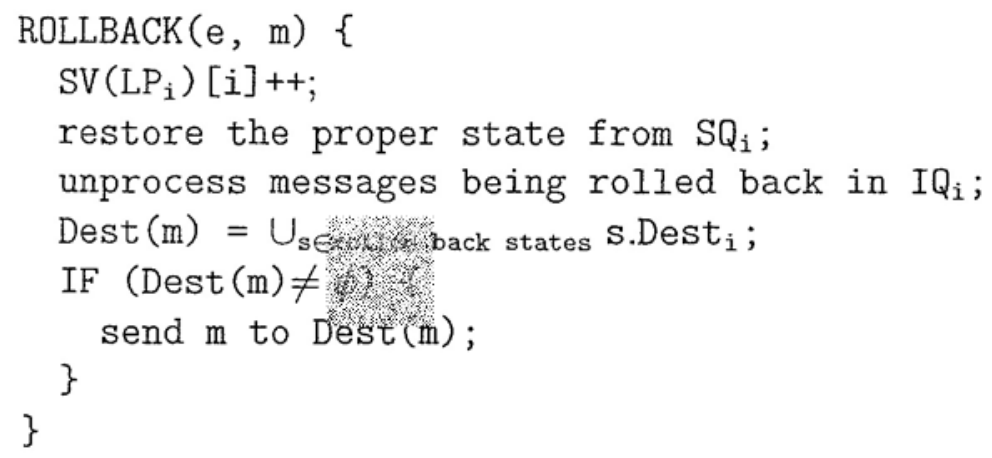

Figure 5.14: Rollback to the state prior to executing e at $L P_{i}$

Figure 5.15 shows a possible scenario applying the batch based cancellation scheme to the scenario in Figure 5.5. Upon receipt of the straggler message at $L P_{1}$, the set of events to be cancelled at $L P_{1}$ is determined as $C_{1}\left(m_{s}\right)=\left\{e_{11}, e_{12}, e_{13}\right\} \quad\left(\right.$ or $\left.\left\{e_{1,5}, e_{1,12}, e_{1,15}\right\}\right)$. 


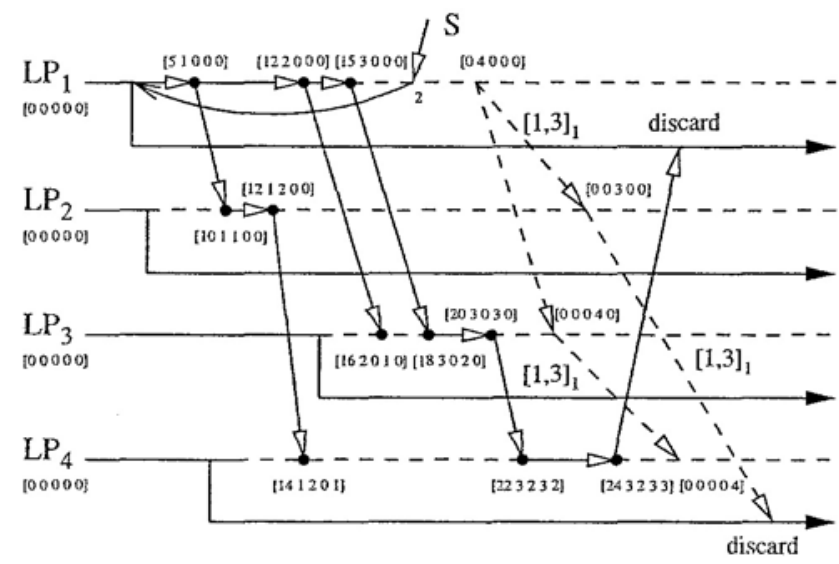

Figure 5.15: Batch based cancellations triggered by a straggler message

Range $[1,3]_{1}$ is sent along with a multicast CANCEL_MESSAGE to $L P_{2}$ and $L P_{3}$. Upon receipt of the range, $C_{2}\left(m_{s}\right)=\left\{e_{21}, e_{22}\right\}$ (or $\left\{e_{2,10}, e_{2,12}\right\}$ ) and $C_{3}\left(m_{s}\right)=\left\{e_{31}, e_{32}, e_{33}\right\}$ (or $\left.\left\{e_{3,16}, e_{3,18}, e_{3,20}\right\}\right)$ are determined respectively and the range is further forwarded to $L P_{4}$ independently by $\mathrm{LP}_{2}$ and $L P_{3}$. The earlier range received by $L P_{4}$ triggers the cancellation of $C_{4}\left(m_{s}\right)=\left\{e_{41}, e_{42}, e_{43}\right\}$ (or $\left\{e_{4,14}, e_{4,22}, e_{4,24}\right\}$ ). The later received one is found to be a duplicate and is discarded.

\subsection{Performance Evaluation}

\subsubsection{Benchmark Model}

A generic manufacturing model [84] (see Section 2.3.3) was used to evaluate the proposed scheme. In order to focus on the demonstration of the advantages of the batch based cancellation scheme in doing fast cancellation and reducing rollbacks, only the part having backward links, i.e., the production line, is used in the benchmark.

A parallel production line continuously produces quality-guaranteed components, which is a constituent part of the final product, through a sequence of processing stations (PS) and control stations (CS) (See Figure 5.16). PS and CS are always paired. A CS is used 
to ensure that no flaw is introduced in the component by its previous PS. If such a flaw is detected, the component is sent to a repair station, (RS) for repairing and then to the PS for reprocessing. In order to better ensure the quality of the component, each component is also tested at the end of its production line after successfully going through each CS. In case of malfunction, the defective component is wholly reworked and sent back to the first PS through an RS. For the sake of clarity, a forker (F) connector is used in production lines to interconnect various stations. A forker routes an input to several output links with configurable probability, which provides a straightforward way to control the reprocessing probability of each component by configuring backward routing probability. Note that there is a simplified forker (without output link) at the end of the production line to represent the endpoint of the simulation (see Figure 5.16 and Figure 5.21).

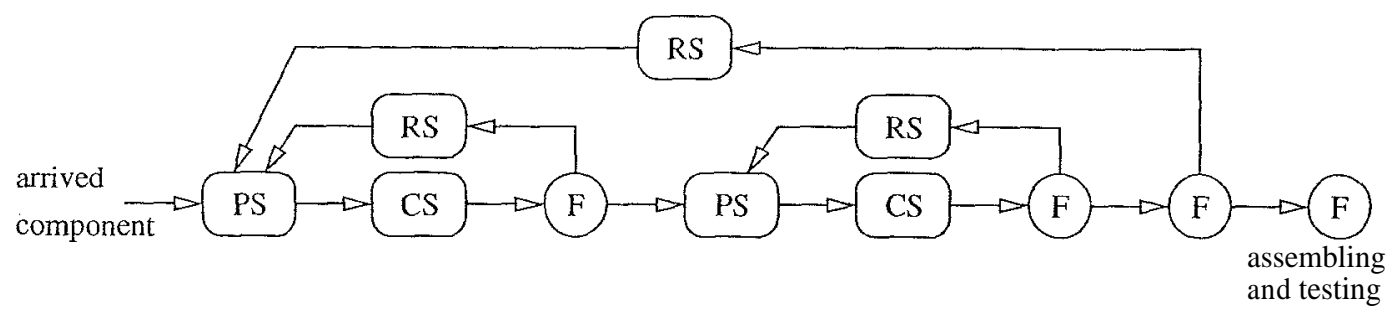

Figure 5.16: Production line

\subsubsection{Performance Metrics}

We define the performance metrics that are used to evaluate the proposed batch based cancellation mechanism over traditional per-event based cancellation scheme. Let $S E$ denote the benchmarked simulation executive. $S E$ can be $T T W, B T W$ or $B T W o p t$, which stand for our implementation of traditional Time Warp simulation executive, batch based Time Warp simulation executive and batch based Time Warp simulation executive with optimization (discussed in Section 5.6.4) respectively. 
- Execution time, denoted as $\operatorname{ExecTime}(S E)$, is defined as the total wall-clock time that the simulation takes to finish using the simulation executive $S E$.

- The total number of simulated events, denoted as TotalEveats(SE),refers to all the events, including committed, rolled back and annihilated/discarded in the simulation.

- The total number of committed events, denoted as CornmittedEvents(SE), counts only committed events in the simulation.

- Efficiency is calculated as below. It reflects the portion of useful computation in the simulation.

$$
\text { Efficiency }(S E)=\frac{\text { CommittedEvent }(S E)}{\text { TotalEvent }(S E)}
$$

- The total number of straggler messages, denoted as Stragglers(SE),counts all the straggler messages received in the simulation.

- The total number of rollbacks, denoted as Rollbacks(SE), is the sum of the times of rollbacks in the simulation.

- The total number of cancel messages, denoted as CancelMessages( $S E)$, is the total number of cancel messages (anti-messages) in the simulation.

- The total number of discarded messages, denoted as DiscardedMessages(SE), is the total number of the positive messages that are detected to be eventually cancelled based on earlier received ranges and, therefore, discarded upon receipt. As discussed before, only BTW and BTWop support the above mentioned functionalities. 
- Normalized speedup measures the speedup of simulating the benchmark model using executive SE over traditional Time Warp simulation executive TTW.

$$
\operatorname{NormalizedSpeedup}(\operatorname{SE}, T T W)=\frac{\operatorname{ExcecTime}(T T W)}{\operatorname{ExecTime}(\operatorname{SE})}
$$

\subsubsection{Experiments}

The experiments were carried out on a Dell 2650 Server, a cluster of 11 nodes (dual 2.6GHz Xeon CPUs, 1GB RAM) interconnected through myrinet and running MPI-GM.

Without loss of generality, the production line for the benchmark has the similar layout as shown in Figure 5.16 and is configured with 4 PSs, 4 CSs, 5 RSs and 6 Forkers. All these 19 units (stations and connectors), i.e., 19 LPs, are one-to-one mapped on 19 CPUS.

Two sets of experiments were carried out with different settings of event granularity and each set reports the comparison between TTW, BTW and BTWopt with different reprocessing probabilities. In each run, 10,000 components are fed to the production line. Table 5.1 and Table 5.2 show the performance data obtained using fine and coarse event granularity respectively. Note that except $\operatorname{ExecTime}(S E)$, all other performance data are the sum of those collected from all the LPs.

In this section, we only conduct comparison between TTW and BTW. The comparison between BTW and BTWopt is given in Section 5.6.4.

Studying the performance data reported from the experiments, we have the following observations.

- For any reprocessing probability, BTW committed similar number of events as TTW did, which meant similar outcome of the simulations regardless underlying simulation executives. The reason for the small difference for a particular reprocessing 


\begin{tabular}{|c|c|c|c|c|c|c|c|c|}
\hline $\begin{array}{l}\text { Simulation } \\
\text { Executive }\end{array}$ & $\begin{array}{l}\text { Reprocessing } \\
\text { Probability }\end{array}$ & $\begin{array}{c}\text { Total } \\
\text { Events }\end{array}$ & $\begin{array}{c}\text { Committed } \\
\text { Events }\end{array}$ & Stragglers & Rollbacks & $\begin{array}{c}\text { Cancel } \\
\text { Messages }\end{array}$ & $\begin{array}{c}\text { Discard } \\
\text { Messages }\end{array}$ & $\begin{array}{c}\text { ExecTime } \\
\text { (ms) }\end{array}$ \\
\hline \multirow{3}{*}{ TTW } & 0.05 & 1745692 & 1441658 & 36605 & 81951 & 219177 & 0 & 26848 \\
\hline & 0.10 & 2293238 & 1488974 & 123637 & 387998 & 566617 & 0 & 36011 \\
\hline & 0.20 & 5362227 & 1581888 & 398195 & 1730366 & 3056360 & 0 & 70473 \\
\hline \multirow{3}{*}{ BTW } & 0.05 & 1661372 & 1438928 & 9715 & 55292 & 52268 & 3080 & 32769 \\
\hline & 0.10 & 1880274 & 1489454 & 20392 & 95284 & 91070 & 6264 & 36024 \\
\hline & 0.20 & 2539777 & 1596774 & 60815 & 241959 & 236787 & 18831 & 54940 \\
\hline \multirow{3}{*}{ BTWopt } & 0.05 & 1591956 & 1439492 & 12495 & 38935 & 63918 & 12264 & 31680 \\
\hline & 0.10 & 1830095 & 1485742 & 18468 & 77077 & 86438 & 24557 & 36077 \\
\hline & 0.20 & 2386219 & 1580156 & 38403 & 152078 & 218062 & 40168 & 50273 \\
\hline
\end{tabular}

Table 5.1: Running the simulation with fine event granularity

\begin{tabular}{|c|c|c|c|c|c|c|c|c|}
\hline $\begin{array}{l}\text { Simulation } \\
\text { Executive }\end{array}$ & $\begin{array}{l}\text { Reprocessing } \\
\text { Probability }\end{array}$ & $\begin{array}{l}\text { Total } \\
\text { Events }\end{array}$ & $\begin{array}{c}\text { Committed } \\
\text { Events }\end{array}$ & Stragglers & Rollbacks & $\begin{array}{c}\text { Cancel } \\
\text { Messages }\end{array}$ & $\begin{array}{c}\text { Discard } \\
\text { Messages }\end{array}$ & $\begin{array}{c}\text { ExecTime } \\
\text { (ms) }\end{array}$ \\
\hline TTW & $\begin{array}{l}0.05 \\
0.10 \\
0.20\end{array}$ & $\begin{array}{l}1682590 \\
2238655 \\
5321969\end{array}$ & $\begin{array}{l}1440540 \\
1488054 \\
1583304\end{array}$ & $\begin{array}{l}33815 \\
117989 \\
384690\end{array}$ & $\begin{array}{l}78025 \\
364046 \\
1672515\end{array}$ & $\begin{array}{l}158680 \\
514483 \\
3015001\end{array}$ & $\begin{array}{l}0 \\
0 \\
0\end{array}$ & $\begin{array}{l}76912 \\
110710 \\
254335\end{array}$ \\
\hline BTW & $\begin{array}{l}0.05 \\
0.10 \\
0.20\end{array}$ & $\begin{array}{l}1613701 \\
1863887 \\
2473967\end{array}$ & $\begin{array}{l}1439684 \\
1488456 \\
1591404\end{array}$ & $\begin{array}{l}7251 \\
25071 \\
56358\end{array}$ & $\begin{array}{l}40506 \\
115671 \\
215067\end{array}$ & $\begin{array}{l}38474 \\
109907 \\
208909\end{array}$ & $\begin{array}{l}2797 \\
5560 \\
14694\end{array}$ & $\begin{array}{l}83279 \\
91924 \\
119431\end{array}$ \\
\hline BTWopt & $\begin{array}{l}0.05 \\
0.10 \\
0.20\end{array}$ & $\begin{array}{l}1585505 \\
1813922 \\
2346895\end{array}$ & $\begin{array}{l}1438976 \\
1488082 \\
1580486\end{array}$ & $\begin{array}{l}10566 \\
18938 \\
35943\end{array}$ & $\begin{array}{l}32786 \\
58764 \\
108008\end{array}$ & $\begin{array}{l}54087 \\
97384 \\
182318\end{array}$ & $\begin{array}{l}11791 \\
21414 \\
38752\end{array}$ & $\begin{array}{l}79065 \\
88411 \\
108328\end{array}$ \\
\hline
\end{tabular}

Table 5.2: Running the simulation with coarse event granularity

probability is because of the variance in random number generation.

- BTW results in a significant reduction of the number of cancel messages (antimessages) in the simulations. This is due to the ability to cancel multiple messages in BTW, as compared with cancelling messages on per-event basis in TTW.

- A smaller number of straggler messages is always achieved in BTW. This can be explained by the rules in Figure 5.8 and Theorem 5.4.3. There are two major reasons for this reduction. One is as shown in Figure 5.16, each PS may receive a repaired component from the corresponding RS, which probably carries smaller time-stamp and causes rollback at the PS. Once a PS rollbacks upon such component, any component to be cancelled and yet to arrive at the PS through the RS will be discarded in the future, avoiding unnecessary processing of possible stragglers. The other reason is that the event processing rules in LPs in BTW introduce more conservatism into the advancement of LPs, in which straggler is less likely to happen. 


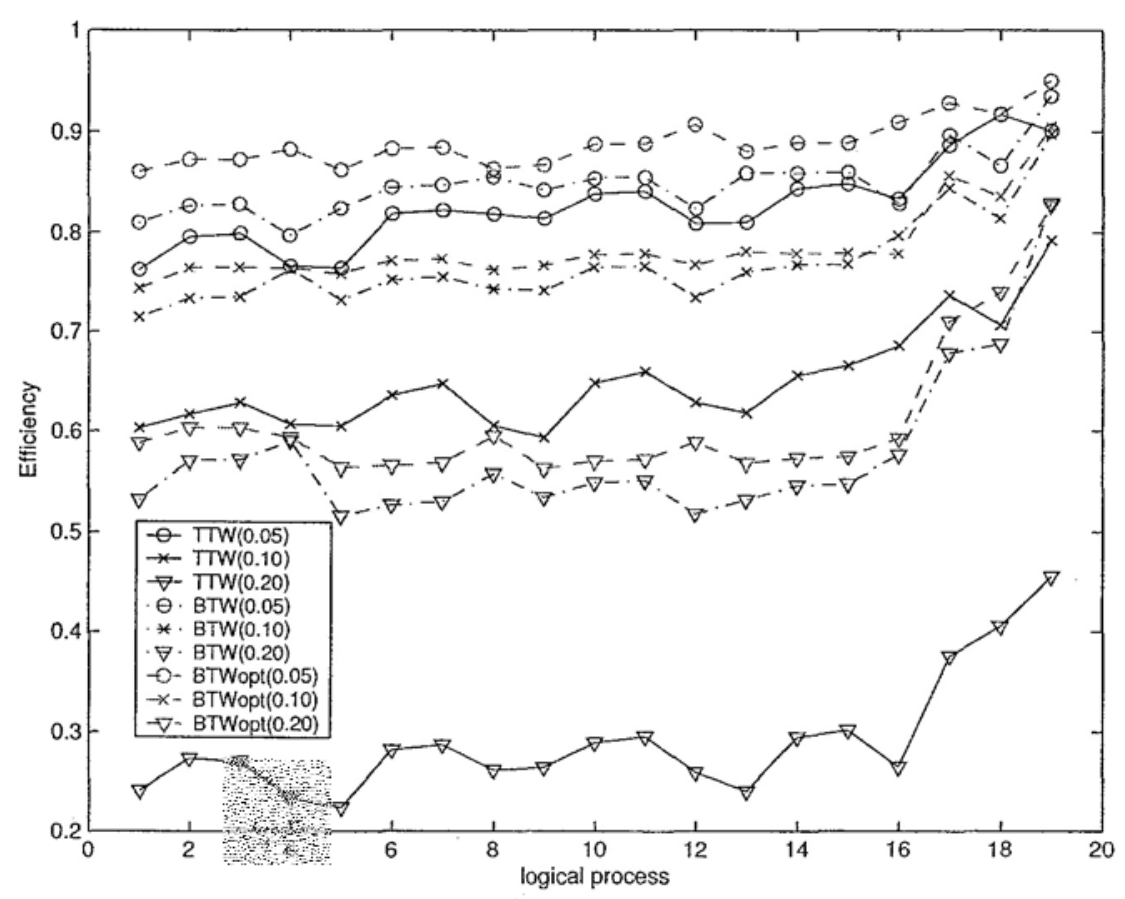

Figure 5.17: Efficiency of individual LPs running with fine event granularity

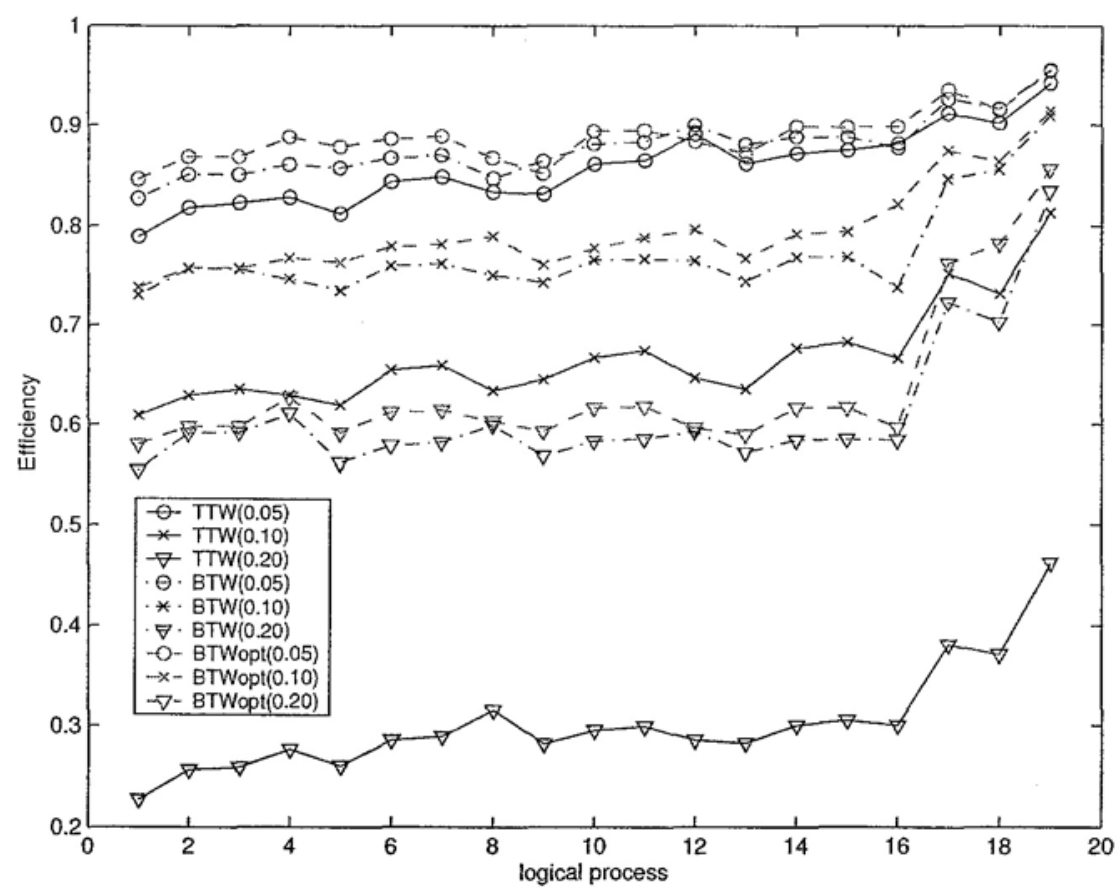

Figure 5.18: Efficiency of individual LPs running with coarse event granularity 
- Less stragglers, less cancel messages and the ability to discard messages that are eventually to be cancelled in the future result in less rollbacks in BTW in all cases. Given similar number of committed events in TTW and BTW, because LPs in BTW process less total number of events, they normally have higher efficiency. Efficiency of individual LPs in TTW and BTW measured from the experiments are shown in Figure 5.17 and Figure 5.18.

- BTW has better efficiency than TTW as shown in Figure 5.19. It can be seen that, using either executive running with either event granularity, efficiency is roughly reversely proportional to reprocessing probability in our experiments. This is due to the fact that the higher number of reprocessed components returned through RSs, the more rollbacks at PSs. This in turn causes more events to be cancelled and reprocessed. It is also obvious that efficiency of TTW degrades much faster than that of BTW as reprocessing probability increases (TTW drops about 65\% while BTW drops about 28\% when reprocessing probability increases from 0.05 to 0.20 .). This illustrates the advantage of BTW over TTW in preventing chasing hazard and cascading rollbacks from happening (chasing hazard and cascading rollbacks at PSs are expected to happen more often with higher reprocessing probability).

Normalized speedup of BTW is shown in Figure 5.20. When the reprocessing probability is as low as 0.05, NormalizedSpeedup $(B T W, T T W)<1$, which means that, in spite of higher efficiency, BTW could perform worse than TTW. This can be explained by the overheads and conservatism introduced by BTW. In TTW, a major factor that affects the performance is the number of rollbacks and reprocessing of events experienced by LPs; whereas in BTW, the batch based cancellation scheme works at the cost of piggybacking more information along with messages and doing more computations at the time of receiving and processing messages (e.g., an LP validates if a message is safe to be processed and updates the message's protocol information). When the reprocessing 


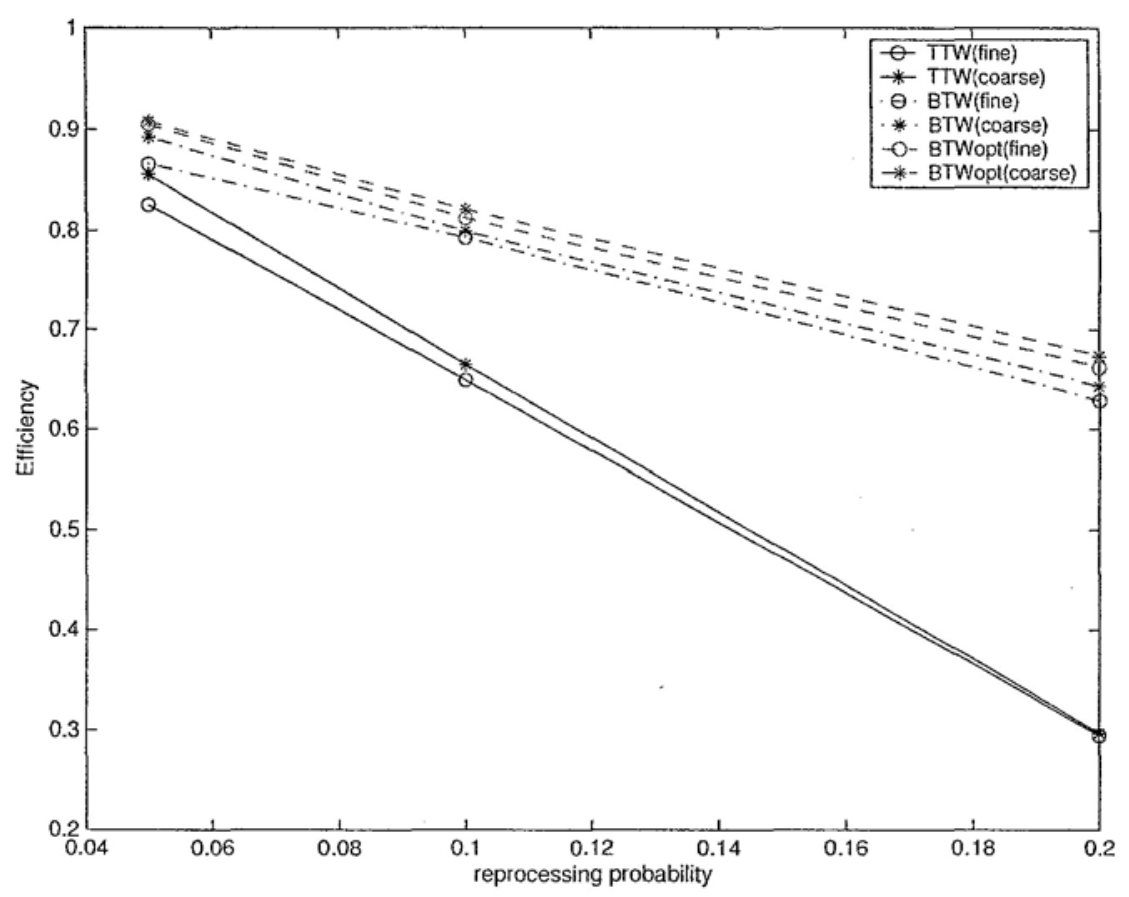

Figure 5.19: Efficiency comparison

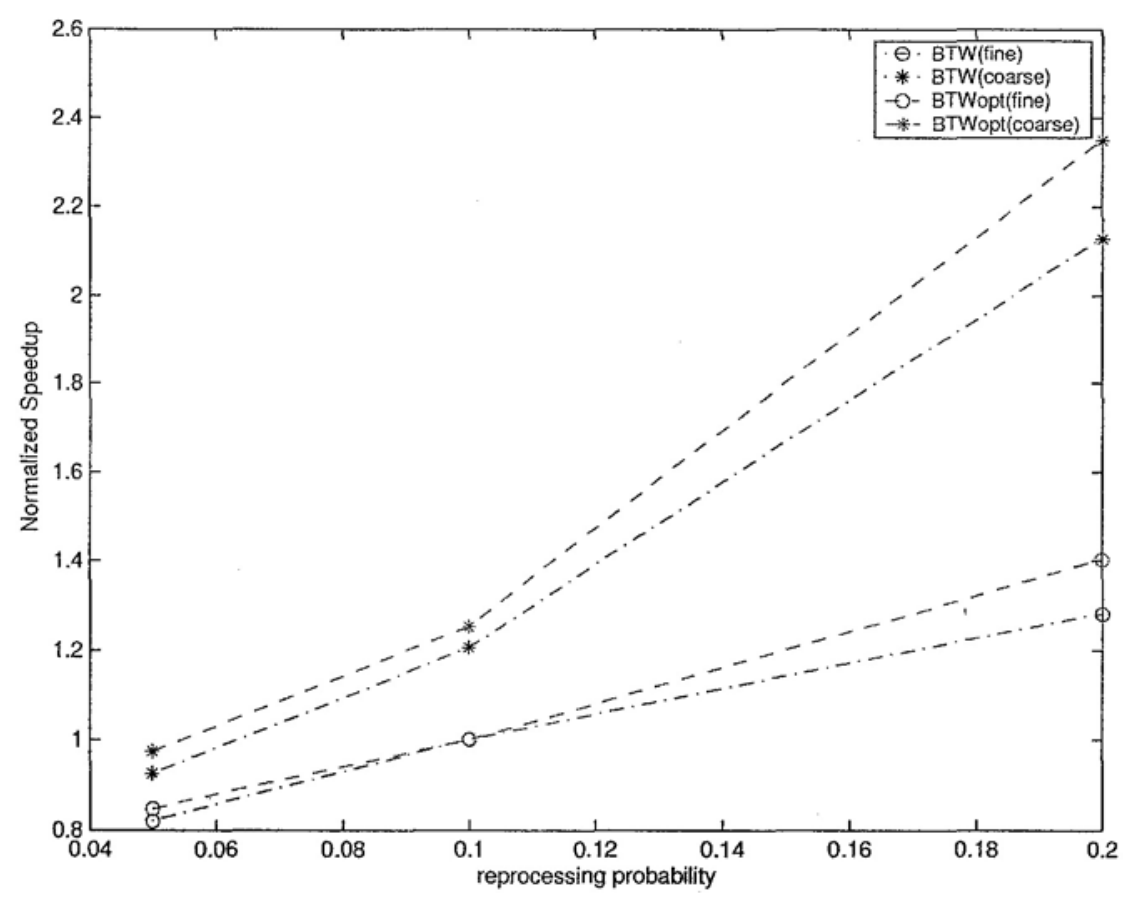

Figure 5.20: Normalized speedup of BTW, BTWopt 
probability is relatively low, TTW is capable of performing better since the likelihood of rollback is small. However, as the reprocessing probability increases, the likelihood of rollback also increases. In this case, the ability to perform the rollback optimal cancellation is crucial to the performance.

It is known that coarse event granularity likely introduces "throttling" effect in a simulation and helps improve its performance. Figure 5.19 shows that this effect is small in our experiments, since efficiency is always similar for a particular reprocessing probability in each executive, regardless event granularity. This shows that batch based cancellation scheme is the major reason for the improvement of performance. However, granularity has effect on normalized speedup. As shown in Figure 5.20, with the same reprocessing probability, better normalized speedup can be obtained in BTW with coarse event granularity.

\subsubsection{Optimizations}

In this section, we discuss a straightforward optimization to the batch based cancellation scheme.

The significance of Theorem 5.4.3 is that the events to be cancelled, either having been scheduled or yet to be scheduled, upon receipt of a straggler message are fully determined by the rollback originator and represented by a range. By expediting the knowledge of this range to other LPs, it is possible to further prevent chasing hazard and, as the result, increase the performance.

This idea is used in the benchmarking model and explained in Figure 5.21. In addition to the communication channels, denoted as solid arrows, in the model, several extra channels, namely, forwarding links, denoted by dashed arrows, are established for the purpose of expediting CANCEL_MESSAGES only (forwarding links are not applicable 


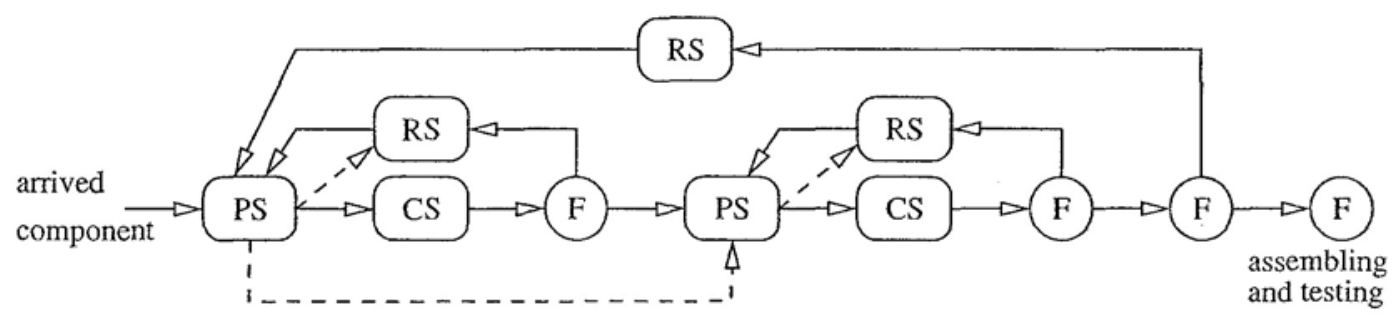

Figure 5.21: Production line with extra forward links

in per-event based cancellation since the dependency on straggler messages cannot be captured using simulation time only). As shown in the figure, a PS sends out a CANCEL_MESSAGE not only to the paired CS, but also to the corresponding RS and the next PS in the simulation path. The advantage of doing so is that the propagation of wrong computations can be stopped at an earlier time without involving more LPs.

For example, suppose at a certain wall-clock time, the first PS receives a repaired component event from an RS and this event causes a rollback. According to the batch based cancellation scheme and the model, a range is immediately generated and propagated to the corresponding CS, RS and the next PS respectively. Without forwarding links, it can be seen that the cancellation can only be done in the regular way: the CANCEL_MESSAGE chases the affected events along with the simulation path until all of them have been eliminated. Although each LP is guaranteed to be rolled back only once, the cancellation might be still inefficient due to the chasing effect. With the help of forwarding links, it is expected that the chasing effect can be reduced by intercepting and discarding most affected events at the RS and the next PS before propagating them further, thus the rest of the LPs in the simulation path are possible to advance more efficiently.

Our benchmarking of the model shown in Figure 5.21 using BTW is referred to as BTWopt, whose performance data have been shown together with those of TTW and BTW in Table 5.1 and Table 5.2. The number of discarded messages reflects the major 
difference of using BTWopt. It is obvious that DiscardedMessage $(T T W)=0$ in all cases, since the cancellation in TTW is done using anti-messages, which only act on their counterparts. DiscardedMessage(BTW) has non zero values, which are the sum of the messages discarded at all the stations. DiscardedMessage(BTWopt) has even greater value, because the forwarding links enable RSs and PSs to discard more affected events. Figure 5.17 through Figure 5.20 show that the increasing number of discarded messages further improves efficiency of processing messages in BTWopt, which accounts for the improvement of speedups over BTW.

It is noted that the introduction of forwarding links into a simulation is very flexible and, with general supportive routines within the simulation executive, can be fully customizable at simulation application level. This flexibility gives simulation developers the freedom to fine tune and optimize performance of a particular real simulation application without any need to tackle underlying executives. One extreme case is to broadcast CANCEL_MESSAGES in the Simulation, i.e., forwarding links are established between any two of LPs. However, it is generally a poor choice given a big number of LPs. One major cost of increasing the number of forwarding links is the potential increase of the number of cancel messages in the simulation (As shown in Table 5.1 and Table 5.2, CancelMessages(BTWopt) is bigger than CancelMessages(BTW)when the reprocessing probability equals to 0.05 .). The other major cost is that, as more ranges are to be checked against, the processing time of individual event also increases, which could degrade the performance. As the rule of the thumb, forwarding links should be considered for the LPs on the critical path of the simulation, which relies upon the characteristics of real simulation applications. 


\subsection{Summary}

In this chapter, a rollback optimal cancellation scheme, the batch based scheme, is presented. Rollback optimal means that any LP is able to recover from the receipt of a straggler message at the cost of at most one rollback. To do this, a state vector is used to capture the dependence of events and a rollback history is utilized to regulate the advancement of LPs and discover at an earlier stage any possible events to be eventually cancelled. We theoretically prove that in the batch based cancellation scheme, the set of events to be cancelled by a straggler message is fully deterministic in terms of the range generated by the rollback originator.

The proposed scheme is feasible in most Time Warp simulations because the scheme only requires additional information piggybacked on messages, additional local structures maintained by LPs and more conservative event processing rules, which are generally adoptable for other Time Warp simulation variants.

From the experiments, we show that the batch based cancellation scheme is able to beat traditional per-event based cancellation scheme in situations where chasing hazards, cascading rollbacks and multiple rollbacks prevail, which meets our research goal to reduce their impact on the performance.

Thanks to the determinism of the events to be cancelled, we also show that, given specific knowledge about the real simulation model, better performance could be further obtained by introducing forwarding links.

The major overheads of the batch based cancellation scheme include the communication cost of the additional state vector and increment of rollback histories carried by positive messages, the storage cost of maintaining rollback history tables and increased computation cost of looking up into and fossil collecting rollback history tables. With the assumption of FIFO channels, the compression approach for the rollback history is 
also applicable to the state vector. Observing that an LP in a simulation normally only communicates with a small number of LPs, the rollback history table at the LP can be maintained within a reasonable amount of space. Currently, the rollback history is simply implemented as a linear list of ranges, and each time an LP receives or processes an event, the list has to be fully scanned. Rollback histories can be organized more efficiently so as to provide more optimized lookup. 


\section{Chapter 6}

\section{Causal order based Time Warp}

\subsection{Introduction}

Analytical parallel and distributed simulation requires that the advancement of each LP ultimately obeys the rule known as the local caudity constraint (LCC) (see Definition 2.3.4), i.e., events are processed in non-decreasing time-stamp order (TSO). To achieve this goal, a considerable number of synchronization algorithms have been proposed and applied, and they roughly fall into two categories, namely, conservative synchronization and optimistic synchronization, which are fully surveyed in Chapter 2. Conservative synchronization strictly prohibits any violations of LCC using well defined protocols $[17,24]$. An LP is allowed to process a certain future event and advance its simulation time only if it is safe to do so. Optimistic synchronization, specifically Time Warp, risks potential violations of LCC by allowing LPs to process future events in an aggressive way. Once a violation really happens, it is detected at runtime and recovery mechanisms are employed accordingly to cancel wrong computations to guarantee LCC.

Through two decades of research on Time Warp simulations, it is known that the optimistic advancement of LPs is one of the keys to improve simulation performance by exploiting more parallelism in event execution. It is also known that over optimistic 
advancement, however, may turn to hazards to the performance. In Chapter 5, we have shown those typical performance hazards such as chasing hazard, rollback echoes, cascading rollbacks and unnecessary multiple rollbacks that directly or indirectly relate to rollbacks in Time Warp simulations.

Limiting optimistic advancement to some degree by introducing certain conservatism into LPs has proved to be a feasible way to reduce possible hazards whereas retain the advantage of Time Warp simulations. Readers may refer back to Chapter 2 for a review of existing approaches.

Although batch based cancellation scheme elaborated in Chapter 5 mainly focuses on the cancellation scheme itself, it indeed introduces conservatism into LPs. Recall the rule of event processing at LPs shown in Figure 5.8, processing of an event at an LP is delayed until the LP is confirmed no longer dependent on a rolled back state which the event is already free from. Thus, LPs can achieve higher event processing efficiency.

If batch based cancellation scheme uses an implicit means to introduce conservatism, causal order based Time Warp elaborated in this chapter can be regarded as an explicit way. The principal observation is that in the circumstances where the violation of the local causality constraint is prone to occurring, e.g., the communication is non-FIFO or multicast is prevailing, delivery of events at LPs can be regulated in the way that avoids LCC violations at the best efforts, namely, capturing the causal relation of events and delivering events in the causal order.

Besides our research, the causal order has been employed for different purposes in Time Warp simulation. For example, in solving the simultaneous events problems [59],the causal order is used to break the tie to prevent potential infinite rollbacks from happening and to guarantee a repeatable simulation sequence [122]. To address inefficiencies in Time Warp’s rollback mechanism, the causal order is used to detect dependencies in cascading and inter-related events and carry out range based cancellation, thereby saving a huge 
amount of time spent in futile computations [29] (see Chapter 5).

Although the causal order can help reduce the chances and the cost of rollbacks, it is only used in [29] to eliminate intermediate causes, namely, rollbacks caused by receipt of anti-messages. The root cause of rollbacks, namely, the chances that straggler messages are received, remains untouched. In the presence of non-FIFO channels or multicast communication among LPs, our experiments show that the chances of receiving straggler messages are even higher. In this chapter, we step further to incorporate the causal order into Time Warp, which forms a new Time Warp simulation paradigm, namely, Causal Order based Time Warp (COBTW). With the adherence to the causal order in COBTW, messages delayed and disordered by communication channels can be fully detected. Based on this knowledge, potential rollbacks caused by out of order processing of causally related messages can be eliminated.

\subsection{Motivations}

In a Time Warp simulation, the processing of messages is always carried out in timestamp order of the messages that have been received but have not been processed. On one hand, this aggressive approach exploits the maximum parallelism among events, i.e., events scheduled by messages are executed with less constraints; on the other hand, it risks additional computational efforts in cancelling wrongly scheduled events, i.e., the so-called over optimism. Once the cancellation becomes overwhelming, the advancement of LPs in the simulation could be heavily hindered. It in turn harms the simulation's performance dramatically.

Figure 6.l(a) shows a scenario in which a straggler message is received through a non-FIFO channel. $L P_{1}$ processes events $e_{1,10}$ and $e_{1,20}$ and schedules events $e_{2,15}$ and $e_{2,30}$ by messages $m_{1}$ and $m_{2}$ respectively. Messages $m_{1}$ and $m_{2}$ are causally related, i.e., 


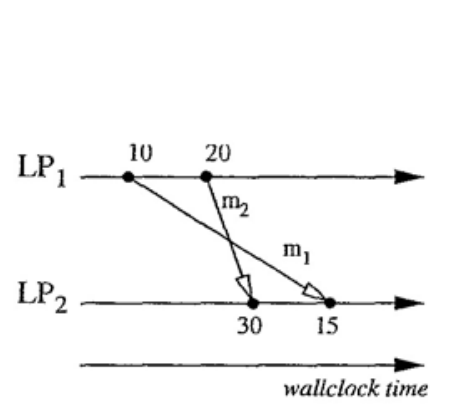

(a) non-FIFO channel

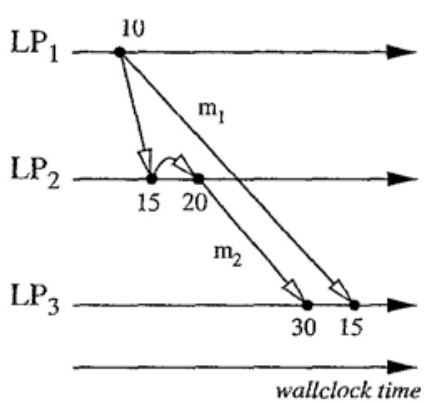

(b) multicast

Figure 6.1: Straggler messages in different environments

$m_{1} \rightarrow m_{2}$, however, they arrive at $L P_{2}$ out of order. When $L P_{2}$ receives $m_{1}$ and processes $e_{2,15}$ scheduled by $m_{1}$, it realizes $m_{1}$ is a straggler message because it has already advanced $\operatorname{LVT}\left(\mathrm{LP}_{2}\right)$ to 30 as the result of processing an earlier received event $e_{2,30}$ scheduled by $m_{2}$. Figure 6.1(b) shows another scenario in which $a$ straggler message is received in a multicast enabled simulation. $L P_{1}$ multicasts a message $m l$ which schedules events $e_{2,15}$ and $e_{3,15}$ at $L P_{2}$ and $L P_{3}$ respectively. Note that it is assumed here that a multicast message always schedules events with identical time-stamps. As a result of processing event $e_{2,15}, L P_{2}$ schedules an internal event $e_{2,20}$ which subsequently schedules another event $e_{3,30}$ and sends it to $L P_{3}$ by message $m_{2}$. It is clear that $m_{1} \rightarrow m_{2}$, but they arrive at $L P_{3}$ out of order due to different channels through which they traverse. Similar to the previous scenario, when $L P_{3}$ receives $m_{1}$ and processes $e_{3,15}$ scheduled by $m_{1}$, it detects $m_{1}$ is a straggler message because it has already advanced its $\operatorname{LVT}\left(L P_{2}\right)$ to 30 as the result of processing $e_{3,30}$ scheduled by $m_{2}$.

Contemporary Time Warp has a straightforward and simple approach in processing incoming messages. It has no preventive measures to detect potential straggler messages as shown in Figure 6.1 to prevent rollbacks from happening. In a distributed simulation, especially the one where LPs communicate via non-FIFO channels with noteworthy delay and jitter or where multicast messages are common, the chances of the above mentioned straggler messages are expected to be high. Once rollbacks occur, due to the complexity 
of the communication pattern, the computational efforts of cancellation are also expected to be significant.

With the help of the causal order, a number of straggler messages could be avoided, thus the number of rollbacks could be reduced. Specifically in Figure 6.1(a)/(b), when message $m_{2}$ is received, $L P_{2} / L P_{3}$ detects that another message, i.e., $m_{1}$ in the figure, that schedules an event with smaller time-stamp is still on its way. Therefore, $m_{2}$ is delayed until $m_{1}$ is received and processed.

Note that COBTW does not change the ultimate processing order of events, namely, the time-stamp order. The causal order can be viewed as away to regulate LPs' optimism by means of checking events' causalities. A violation of the causal order indicates a potential violation of the local causality constraint, during which the specific LP must stop its advancement and wait for the late arrived straggler message(s). Conceptually, there exist discrepancies between the causal order and the time-stamp order. This issue is addressed in Section 6.5.

\subsection{Effective Path of Advancement}

Before introducing the causal order, we first examine the advancement behavior of Time Warp simulations. Diagrammatically, the advancement of LPs can be drawn as a tree diagram [106]. For its importance for understanding the mechanism of COBTW, it is elaborated as follows.

As shown in Figure 6.2, the advancement of each LP in a simulation is viewed as starting a single branch. A new branch ramifies at the point of each rollback. The extent of each branch indicates the distance the LP advances before being rolled back. The black dots denote the events that occur in the simulation. The dashed branches represent the computations which have already been cancelled. The solid branches, with the arrows 
at their right ends, define the effective paths of the advancement (EPA) along which LPs evolve.

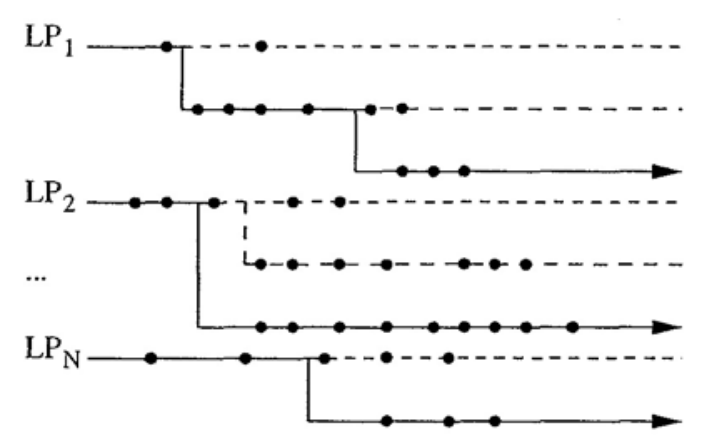

Figure 6.2: The advancement of LPs

It is obvious that, through out the simulation, $L P_{i}$ exactly has one EPA, denoted by $E P A_{i}$. In addition, each LP's EPA is fully undetermined until the simulation terminates due to the dynamical rollback behavior of Time Warp simulations. So, we have the following straightforward observation:

Observation 6.3.1. When a simulation terminates, events on each LP's EPA define its effective computations.

\subsection{Processing Messages in the Causal Order}

To simplify the explanation of the COBTW mechanism, the straightforward implementation [118] of the causal order is adopted and only the handling of external messages, i.e., basic and protocol messages that were not destined to the sender LP itself, is discussed. Internal messages are sent, received and processed in the same way as that in traditional Time Warp.

The clock maintained by $L P_{i}$ is augmented with four components, namely, $L V T\left(L P_{i}\right)$, $V T\left(L P_{i}\right), \operatorname{DELIV}\left(L P_{i}\right)$ and $\operatorname{SENT}\left(L P_{i}\right) . \operatorname{LVT}\left(L P_{i}\right)$ denotes the simulation time of the LP which is the only clock component maintained by traditional Time Warp simulations. 
$V T\left(L P_{i}\right)$ is an $\mathrm{N}$-tuple vector time where $V T\left(L P_{i}\right)[\mathrm{i}]$ denotes the number of external messages that $L P_{i}$ has sent and $V T\left(L P_{i}\right)[j], i \neq j$, denotes $L P_{i}$ 's knowledge of another $L P_{j}$ 's $V T\left(L P_{j}\right)[j] . \operatorname{DELIV}\left(L P_{i}\right)$ is an N-tuple where $\operatorname{DELIV}\left(L P_{i}\right)[j], i \neq j$, represents the number of external messages sent from $L P_{i}$ and processed by $L P_{i} \cdot \operatorname{SENT}\left(L P_{i}\right)$ is an $N \times N$ matrix and $\operatorname{SENT}\left(L P_{i}\right)[j][k]$ shows $L P_{i}$ 's knowledge of the number of external messages sent from $L P_{j}$ to $L P_{k}$ (not necessarily processed). Similarly, the clock carried by an external message $m$ also has three components, namely, $S T(m), V T(m)$ and $S E N T(m) . S T(m)$ is the time-stamp at which the carried event is scheduled to be processed. $V T(m)$ and $S E N T(m)$ are the snapshots of the sending LP's $V T(L P)$ and $S E N T(L P)$ at the time the message is sent out. Among these components, DELIV and $S E N T$ are necessary for the implementation of the causal order delivery. VT is essential to determine the causal order between any two external messages [126].

We implemented a Time Warp simulation executive incorporated with the causal order delivery of external messages, namely, Causal Time Warp (CTW), based on techniques presented in Chapter 4. CTW extends ATW by implementing necessary interfaces. For the seek of clarity, we present at a higher level by selectively elaborating important functional routines instead of presenting individual interfaces. Note that these routines are not assumed one-to-one mapped to the interfaces: implementation of them may involve more than one interfaces.

The updating of $L P_{i}$ 's clock is performed when $L P_{i}$ schedules and processes events via external messages, which are shown in Figure 6.3 and Figure 6.4 respectively, where supreme denotes the componentwise maximum operation. The wait step in Figure 6.4 states that the delivery of message $m$ is made upon all its causally preceding messages, identified by its carried $S E N T$ matrix, having been delivered at the LP by checking with the LP's locally stored DELIV vector. Details about each step can be found in $[118,126]$. 


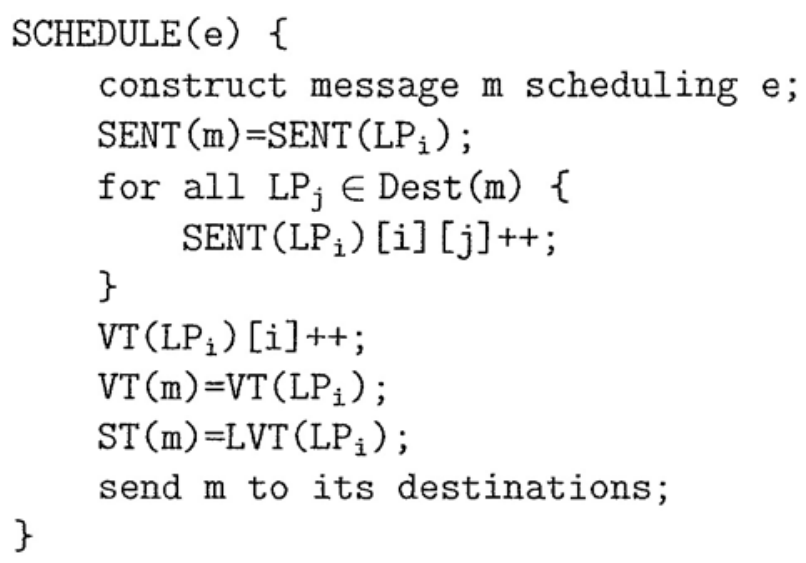

Figure 6.3: Scheduling an external event by $L P_{i}$

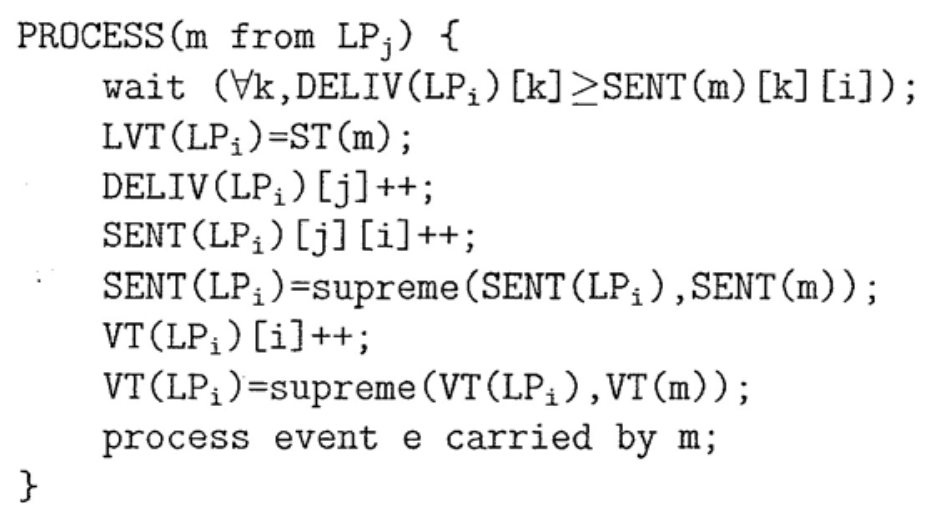

Figure 6.4: Processing an external message by $L P_{i}$

\subsection{Problem of Discrepancies}

\subsubsection{Discrepancies between CO and TSO}

It is apparent that the elements of $V T\left(L P_{i}\right)$ increase monotonically and the sending order of messages determines their causal relation, which subsequently defines their receiving order. In other words, if external messages $m_{1}$ and $m_{2}$ are from the same LP and $m_{1}$ was sent before $m_{2}$, then $m_{1} \rightarrow m_{2}$, which means $m_{1}$ must be processed before $m_{2}$ to be coherent with the causal order. This principle is almost consistent with paradigms in Time Warp simulations, which are shown as Observation 6.5.1.

Observation 6.5.1. Assume that there are no optimizations considered. 
- An anti-message is always sent after and required to be processed after its corresponding positive message.

- A positive message sent again after cancelling its previous sending is always required to be processed after the latter.

However, there are still discrepancies due to the fact that the time-stamp order requires time-stamped messages to be processed in the order of their time-stamps, not necessarily in their sending sequence.

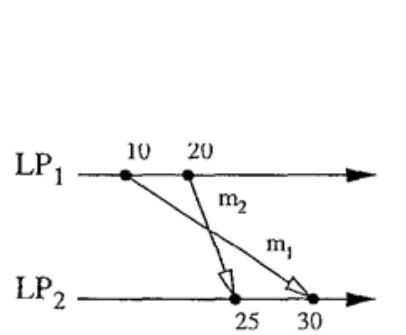

(a)

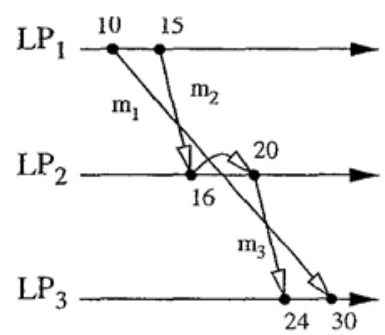

(b)

Figure 6.5: The causal order conflicts with the time-stamp order

Figure 6.5(a) shows a scenario sometimes found in simulations. $L P_{1}$ processes events $e_{1,10}$ and $e_{1,20}$ and schedules events $e_{2,30}$ and $e_{2,25}$ by messages $m_{1}$ and $m_{2}$ respectively. The difference, compared with Figure 6.l(a), is that $L P_{1}$ schedules events in a nonmonotonically ascending way, i.e., $m_{2}$ is sent after $m_{1}$ but schedules an event with smaller time-stamp. Under this circumstance, the causal order inevitably fails because $m_{2}$ is supposed to be processed before $m_{1}$ according to the time-stamp order but forcibly delayed after $m_{1}$ due to the causal order. This kind of failure can exist even if messages are sent to different LPs. In Figure 6.5(b), $L P_{1}$ processes event $e_{1,10}$ and sends $L P_{3}$ message $m_{1}$ with event $e_{3,30} . L P_{2}$ processes $e_{2,16}$ scheduled by $m_{2}$ and schedules an internal event $e_{2,20}$ which subsequently sends $L P_{3}$ message $m_{3}$ with event $e_{3,24}$. It is obvious that $m_{1} \rightarrow m_{3}$. But unfortunately, they cannot be processed in the causal order at $L P_{3}$ for the same reason. 


\subsubsection{Removing Discrepancies at Sending Side: Reordering}

Recalling Observation 6.3.1, solutions to remove the discrepancies are governed by Observation 6.5.2 given below.

Observation 6.5.2. The causal order is said to be consistent with the time-stamp order iff a simulation terminates, for all $i, 1 \leq i \leq N$, the causal order of the external messages processed on $E P A_{i}$ is coherent with the time-stamp order of the events scheduled by those messages. That is, for any two events $e_{i, t_{1}}$ and $e_{i, t_{2}}$ on $E P A_{i}$, scheduled by external messages $m_{1}$ and $m_{2}$ respectively, if $m_{1} \rightarrow m_{2}$, it must hold that $t_{1} \leq t_{2}$.

Observation 6.5.2 conceptually defines an approach to remove the discrepancies between the causal order and the time-stamp order by guaranteeing the coherence among the external messages and the scheduled events which perform effective computations. However, it is empirically hard as we mentioned earlier for these messages and events are generally unknown before the simulation terminates. An alternative approach, namely, reordering, is by guaranteeing the coherence among all the external messages and the scheduled events at any time in a simulation. This can be done locally at each sending LP by ensuring no discrepancies among the outgoing messages and the events it schedules. Before an LP sends an external message $m_{2}$ carrying an event with time-stamp $t$, it always checks its output queue. If there exists an outgoing message $m_{1}$ that carries a larger time-stamped event, a process of reordering takes place. $m_{1}$ must be cancelled first by sending its anti-message and re-sent after $m_{2}$.

The mechanism of reordering can be illustrated using the scenarios in Figure 6.5. As shown in (a), when $L P_{1}$ processes $e_{1,20}, L P_{1}$ cancels $m_{1}$, sends $m_{2}$ and re-sends $\mathrm{ml}$ again to guarantee the consistency. Similarly in (b), when $L P_{i}$ processes $e_{1,15}, L P_{1}$ cancels $m_{1}$, sends $m_{2}$ and re-sends $m_{1}$ again. Because now $m_{1} \| m_{3}$, the discrepancy is removed after the reordering process. 
The correctness of the reordering approach can be proved by the transitiveness of the causal order and one of the basic characteristics of Time Warp simulations, i.e., an LP always schedules events in its future. Suppose there are two events $e_{i, t_{1}}$ and $e_{i, t_{2}}$ scheduled at $L P_{i}$ by messages $m_{1}$ and $m_{2}$, where $m_{l} \rightarrow m_{2}$. It holds that $t_{1} \leq t_{2}$ according to the above mentioned sending scheme, because along a chain of intermediate causally related messages between $\mathrm{m}_{1}$ and $m_{2}$, the time-stamps of scheduled events are always guaranteed to be increased monotonically.

The drawback of the reordering approach, however, is the introduction of additional rollbacks brought about by the removal of discrepancies between the causal order and the time-stamp order. Compared with the same paradigms in Time Warp simulations, the approach incurs one additional sending of $m_{1}$ and its anti-message in both Figure 6.5(a) and (b). It seems paradoxical to the ultimate goal of the introduction of the causal order, removing unwanted rollbacks.

\subsubsection{Removing Discrepancies at Receiving Side: Eliminate Re- ordering}

The reordering approach at sending side presented above strictly preserves the consistence of the causal order and the time-stamp order at the cost of extra reordering. However, if the solution is moved onto the receiving side, our further study reveals that the discrepancies can be eliminated more naturally without introducing extra sending and cancellation of messages.

For any two messages that are parallel to each other, the causal order delivery does not impose any constraint upon their processing order, neither does traditional Time Warp. So, the problem is essentially restricted to the messages having the causal order relation. Table 6.1 summaries different situations in which an LP in traditional Time Warp or COBTW shall behave when receiving causally related messages. Note that the 
paramount rule for COBTW is that the causal order delivery is never breached.

\begin{tabular}{|c|l|l|l|}
\hline Messages' Relation & Arrival Order & \multicolumn{1}{|c|}{ Time Warp } & \multicolumn{1}{c|}{ COBTW } \\
\hline$m_{1} \rightarrow m_{2}$, & $m_{1}, m_{2}$ & deliver $m_{1}$ first, then $m_{2}$. & deliver $m_{1}$ first, then $m_{2}$. \\
\cline { 2 - 4 }$S T\left(m_{1}\right)<S T\left(m_{2}\right)$ & $m_{2}, m_{1}$ & $\begin{array}{l}\text { deliver } m_{2} \text { first, followed } \\
\text { by a rollback, then } m_{1}, m_{2} .\end{array}$ & $\begin{array}{l}\text { delivery of } m_{2} \text { is withheld } \\
\text { until } m_{1} \text { is delivered. }\end{array}$ \\
\hline \multirow{2}{*}{$\begin{array}{c}m_{1} \rightarrow m_{2}, \\
S T\left(m_{1}\right)>S T\left(m_{2}\right)\end{array}$} & $m_{1}, m_{2}$ & $\begin{array}{l}\text { deliver } m_{1} \text { first, followed } \\
\text { by a rollback, then } m_{2}, m_{1} .\end{array}$ & $\begin{array}{l}\text { deliver } m_{1} \text { first, followed } \\
\text { by a rollback, then } m_{2} m_{1 .}\end{array}$ \\
\cline { 2 - 5 } & $m_{2}, m_{1}$ & deliver $m_{2}$ first, then $m_{1}$. & $\begin{array}{l}\text { delivery of } m_{2} \text { is witheld } \\
\text { until } m_{1} \text { arrives. }\end{array}$ \\
\hline
\end{tabular}

Table 6.1: Different behaviors between traditional Time Warp and COBTW when handling causally related messages

It can be seen from the table that there are four situations, which can be interpreted from two aspects. From the aspect of LPs' behavior, two Time Warp paradigms behave differently in two out of four situations, which are further discussed as below.

- $m_{1} \rightarrow m_{2}, S T\left(m_{1}\right)<S T\left(m_{2}\right), m_{2}$ arrives before $\mathrm{m}_{1}$ : This situation probably happens when the communication channel is non-FIFO or multicast transmission is involved (as discussed on Figure 6.1 in Section 6.2), which is expected to be the major advantage of using COBTW.

- $m_{1} \rightarrow m_{2}, S T\left(m_{1}\right)>S T\left(m_{2}\right), m_{2}$ arrives before $m_{1}$ : In this situation, COBTW behaves more conservatively. $m_{2}$ is allowed to be delivered only after $m_{1}$ has been received simply because of the constraint imposed by the causal order. Nevertheless, except for the delay of $m_{2}$, no rollback is required in both paradigms.

From the aspect of discrepancies, understanding of COBTW's behavior in the last two situations is essential for understanding the receiving side approach. In case the discrepancy happens in COBTW, i.e., $m_{1} \rightarrow m_{2}, S T\left(m_{1}\right)>S T\left(m_{2}\right)$, if $m_{1}$ arrives earlier than $m_{2}$, the causal order between $m l$ and $m_{2}$ need not be readjusted at all because the receiving LP will eventually roll back as that in traditional Time Warp. Otherwise, if $m_{2}$ 
arrives earlier, the delay of $m_{2}$ until $\mathrm{m}_{1}$ arrives enables the receiving LP to identify the discrepancy, thus, the LP is able to repair it locally by delivering $m_{2}$ before $m_{1}$.

In summary, LPs in COBTW can behave similarly as those in traditional Time Warp. The above discussion suggests a more elegant way to remove discrepancies, i.e., removing them at receiving side. Compared with the reordering approach at sending side, discrepancies are allowed to occur in COBTW but are repaired as soon as they are detected. Once such a discrepancy is found, it is prevented from being further propagated by a rollback (the third situation) or block and repair (the fourth situation) at the receiving LP. Thus, the rest of LPs can still rely on the causal order delivery. In the implementation of CTW, the approach of removing discrepancies at receiving side is used.

\subsection{The Causal Order based GVT Approximation}

Global Virtual Time (GVT) is computed as the minimal time-stamp of all unprocessed and partially processed messages and anti-messages in a simulation at certain wall-clock time. GVT is essential for the periodical memory reclamation, known as fossil collection, from LPs' working queues. Two major problems, namely, the transient message problem and the simultaneous reporting problem, make the GVT computation naturally challenging [59].

Mattern's GVT approximation algorithm is based on the concept of consistent cut, which divides each LP in the simulation into past and future parts and guarantees that there are no messages sent from the future of the sending LP to the past of the receiving LP. In practice, the construction of consistent cut is non-trivial because of the difficulty of determining the set of transient messages. In order to do this without using message acknowledgements, Mattern's algorithm is based on two different cuts as shown in Figure 6.6. 


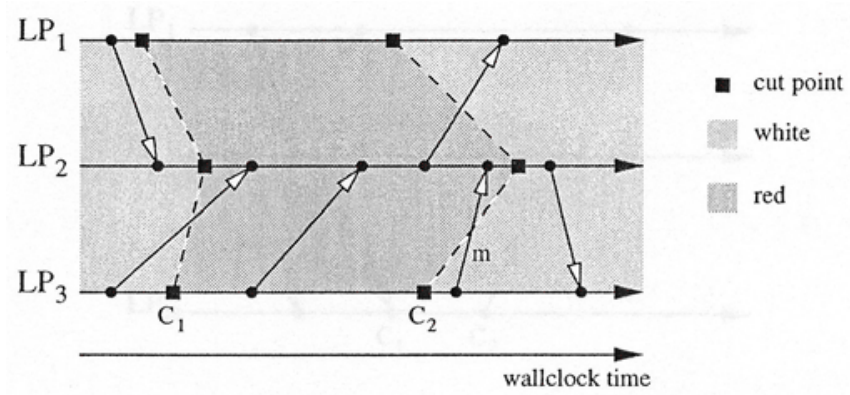

Figure 6.6: Mattern's GVT approximation

The first cut, denoted as $C_{1}$, is a consistentcut. It is used to notify each LP, upon reaching its cut point, to start to record the smallest tirne-stamp of any rnessages it sends. The second cut, denoted as $C_{2}$, is used to guarantee that any messages sent before $C_{1}$ have been received before the receiving LP reaching its cut point. Put in another way, the essentiality of $C_{2}$ is to ensure that all the transient messages sent before $C_{1}$ are captured completely. Note that $C_{2}$ is not a consistent cut. As shown in the figure, message $m$ sent from $C_{2}$ 's future to its past could exist. However, it does not affect the correctness of the algorithlm since $S T(m)$ is at least as large as the computed GVT [47, 59].

The classic implementation of Mattern's GVT approximation algorithm is by coloring LPs in the simulation. All the LPs are initially in white color. Upon reaching its cut point of $C_{1}$, the LP changes its color to red and remains until it reach its cut point of $C_{2}$. A message sent between two LPs is colored as that of its sending LP's. So, GVT is computed as the minimum time-stamp of all red messages and all unprocessed messages defined by $C_{2}$.

In the presence of the causal order, a simplified GVT approximation algorithm that works similarly to Mattern's is shown in Figure 6.7. A protocol message, namely, GVT message (GM) which is sent and received in the same way as basic messages in the simulation, is introduced for the approximation. The approximation involves two phases. In the forwarding phase, $L P_{1}$ initially sends a GM to $L P_{2}$ and then $L P_{2}$ immediately 


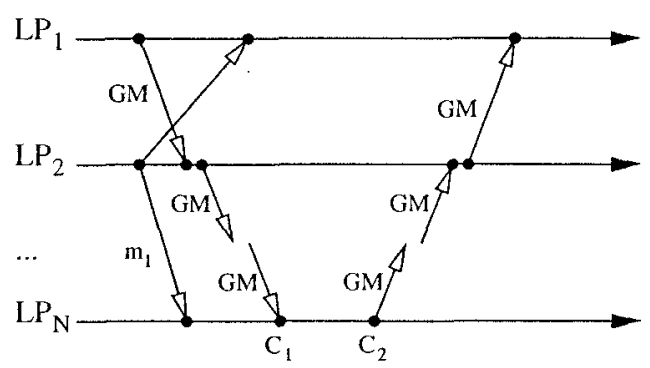

Figure 6.7: The causal order based GVT approximation

forwards it to $L P_{3}$. This procedure continues and this phase ends when the GM arrives at $L P_{N}$. In the backward phase, the GM is sent in the opposite way to that in the forwarding phase and finally reaches $L P_{1}$. The forwarding path and the backward path effectively define two consistent cuts $C_{1}$ and $C_{2}$ respectively. Thus, the computation of GVT along $C_{2}$ can be done in the same way as that in Mattern's GVT approxirnation algorithm. Compared with Mattern's GVT approximation algorithm, the major simplifications in the above approach are in the constructioii of $C_{1}$ and $C_{2}$. Neither color messages nor a distributed termination algorithm is required. Due to the causal order delivery, any messages, e.g., $m_{1}$, sent prior to $C_{1}$ are guaranteed to be received before the receiving LP reaches $C_{2}$.

\subsection{Performance Evaluation}

\subsubsection{Benchmark Model}

To be general, the parallel hold model (PHOLD [58]) (see Section 2.3.3) was adopted in our experiments. PHOLD is a synthetic workload model which does not target any specific real-world simulation but is highly configurable to study various characteristics of a wide range of simulations that could be found in practice. The configurability of PHOLD is fulfilled by six model parameters, which are presented below. 
- The number of LPs: This parameter determines the maximum availability of parallelism in the simulation.

- Message population: It is the number of "seed" events initially placed in the simulation. Once a simulation starts, it is assumed that there is no events "spontaneously" created, i.e., the schedule of any new event hereafter is due to processing of a previously received event. Thus message population essentially defines the number of causality threads migrating through the simulation.

- Time-stamp increment: This is identical to the one found in any Time Warp simulation. Time-stamp increment is allowed to be zero.

- Movement function: It provides customization for the destination of any newly scheduled event.

- Computation granularity: This parameter defines the amount of computations to process a single event. Other overheads incurred in scheduling, fossil collections are excluded.

- Initial configuration: This parameter defines the owner LPs of those "seed" events before the simulation begins.

In our experiments, the last five model parameters were configured. The number of LPs was fixed to 16 and each LP was mapped to a single CPU. To test LPs' different behaviors under different workloads, message population, i.e., the number of event scheduling threads, was varied. Each experiment was repeated with the message population of 2, 4, 8 and 16, which emulates light, moderate, heavy and saturated workloads respectively. The time-stamp increment was dynamically set to a random value uniformly distributed in the range of $[0,10]$. Zero increment is allowed. The event processing time (computation granularity) was set to a random value uniformly distributed in the range of Elms, 10ms]. 
Because all the LPs are assumed to be equally dispersed and fully interconnected, an LP is able to schedule an event to itself or any of other LPs by using the movement function. For the purpose of studying performance under symmetric workload and asymmetric workload, different movement functions are used in the experiments. In the case of multicast of events is used, it is assumed that only one of the scheduled multicast events can subsequently schedule other events to avoid potential exponential increase of messages population which could overload the simulation quickly.

\subsubsection{Experiments}

The experiments were carried out on the same cluster as that used in Chapter 5. The comparison was conducted by running the above presented PHOLD model on the TTW executive and the CTW executive respectively. Among the performance metrics listed in Section 5.6.2, the total number of rollbacks, the efficiency, and normalized speedup of CTW over TTW are of great importance and evaluated from the experiments.

The first set of experiments were carried out using symmetric workload, in which the movement function is simply defined as a uniformly distributed random function. Except for initial configuration, each LP has exactly the same logic and behaves identically throughout the simulation. For this reason, the average number of rollbacks experienced by an LP, denoted by Rollbacks $(L P)$, and the average efficiency of an LP, denoted by Efficiency $(L P)$,are used to measure the rollbacks experienced by any LP and efficiency of any LP in the simulation. Intuitively, Rollbacks $(L P)$ reflects the average number of times an LP rewinds to its previous states and is proportional to the amount of computations being cancelled with the assumption of the aggressive cancellation strategy. Thus, it eventually has effect on the speedup of the simulation.

Figure 6.8 shows the results of the experiments running on the cluster. LPs mapped on the cluster communicate through high speed Myrinet. Moreover, because both TTW 


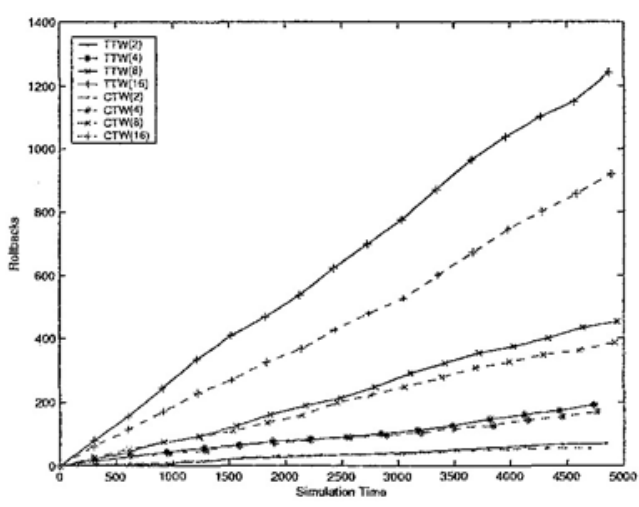

(a) unicast

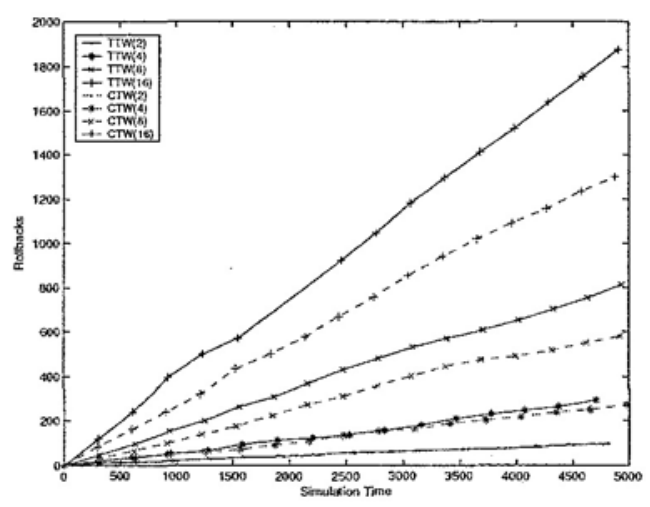

(b) multicast

Figure 6.8: Comparison of RolZback(LP) on cluster (symmetric)

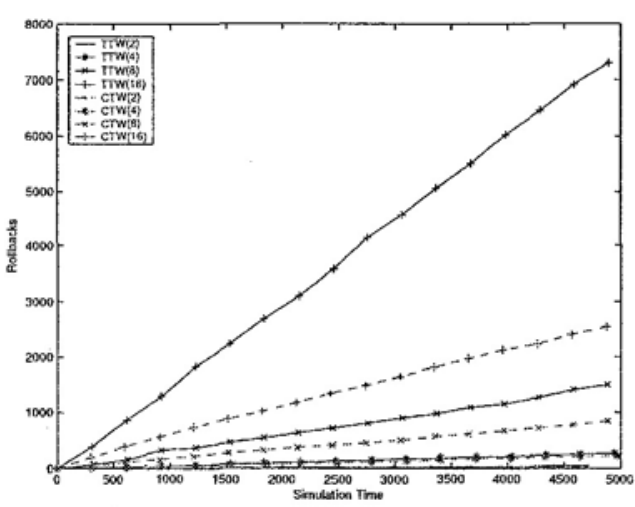

(a) unicast

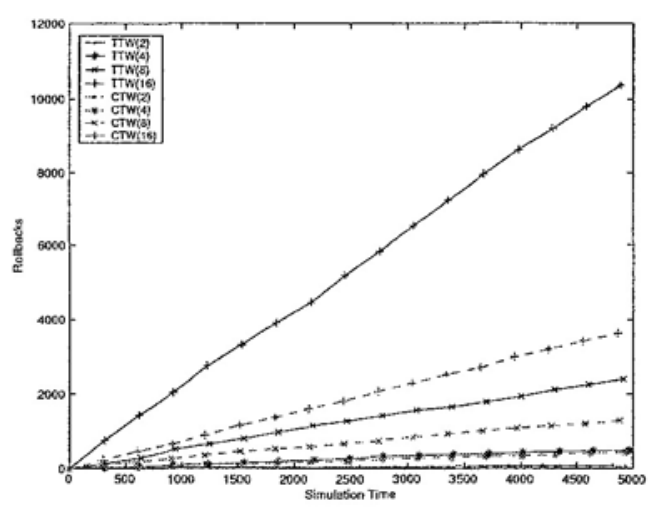

(b) multicast

Figure 6.9: Comparison of Rollback( $L P)$ in emulated WAN (symmetric) 
and CTW executives are built on the top of MPI-GM [53], the transmission between any two LPs is actually in FIFO order. Figure 6.8(a) shows the LPs' average rollback behavior with only unicast enabled. Figure 6.8(b) depicts the rollback behavior with the configuration that the processing of any event has 0.3 probability to schedule multicast events (the number of destinations of each multicast event is fixed at two). It can be seen that TTW and CTW exhibit similar rollbacks under light workload and moderate workload. This is because causal order violation seldom happens when the message population is small. As workload increases, the chances of causal order violation increase as well, in which the advantage of preventing them from happening becomes more obvious for the LPs in CTW. Compared with TTW, Rollbacks $(L P)$ in CTW drops by $15 \%$ and $29 \%$ in unicast environment and multicast environment respectively under heavy workload and $26 \%$ and $31 \%$ respectively under saturated workload. Note that there is a significant increase of Rollbacks( $L P)$ drop rate (from 15\% to 26\%) between unicast and multicast under heavy workload. This shows that causal order violations more likely happen in multicast environment with higher message population and CTW proves its ability to reduce excessive rollbacks and maintain the simulation in a stable state.

Figure 6.9 shows the results of the experiments running in an emulated WAN. To emulate the latency of WAN (normally in the order of milliseconds [7]) and the non-FIFO channels, the network emulation module presented in Section 4.2 is used. Any outgoing message is locally buffered for a random time uniformly distributed in the range of [50ms, 150ms] before being sent through the underlying communication channels. Because of the non-FIFO property, it can be seen that a significant increase of the number of rollbacks were experienced by both executives. Nevertheless, the advantage of using CTW is more obvious. Because of the causal order delivery, most rollbacks caused by the non-FIFO channels and multicast transmissions were avoided in CTW and its Rollbacks $(L P)$ is proved to be less sensitive to increasing workload. 
Figure 6.10 summarizes average efficiency in all experiments running with symmetric workload. LPs in CTW have higher average efficiency than those in TTW in all cases. This can be explained by the conservatism introduced by the causal order delivery. There are two sources of the conservatism. The first is increased computing overheads in processing events, which consequently has throttling effect on the simulation. The second is that, according to the causal order delivery, CTW can actively delay the processing of certain events to avoid rollbacks (recall the second situation in Table 6.1).

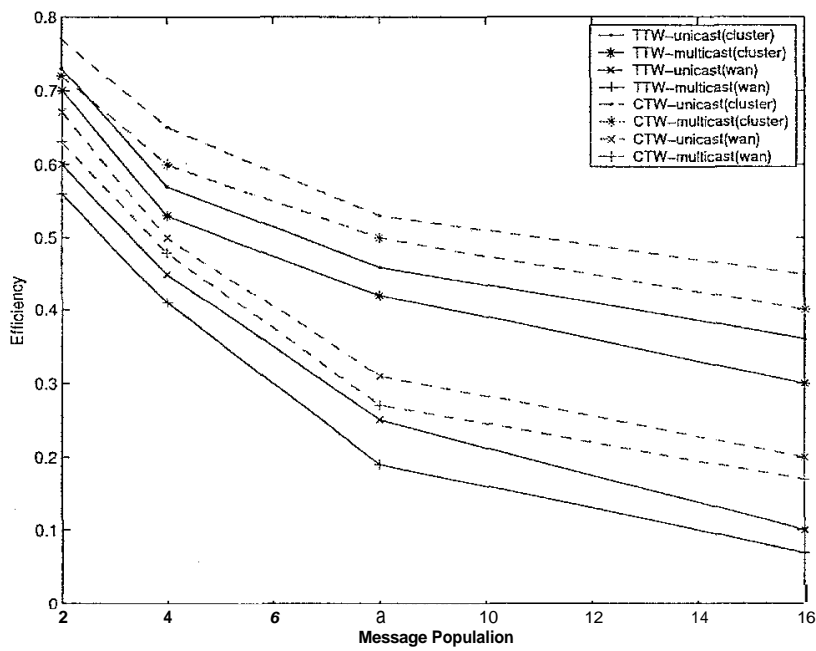

Figure 6.10: Comparison of Efficiency $(L P)$ (symmetric)

The computing overheads required by the causal order delivery, however, has negative impact on the performance of the simulation as well. Figure 6.11 summarizes calculated NorrnalizedSpeedup $(C T W, T T W)$ under symmetric workload. When the workload is low, the performance of CTW is slightly lower than that of TTW in cluster environment because of the existence of computing overheads. As the workload increases and the possibility of causality violations increases, the contribution of the causal order delivery offsets its overhead, which is manifested by that CTW outperforms TTW correspondingly.

The above experiments using symmetric workload are too idealized for they are too homogeneous and can hardly be found in real applications. To further study the merits 


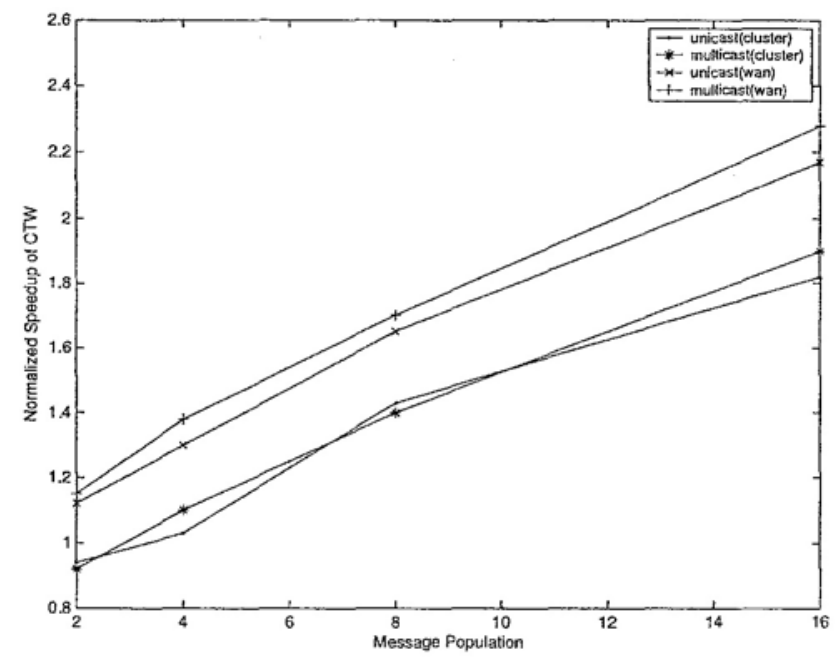

Figure 6.11: Normalized speedup of CTW (symmetric)

of CTW, the second set of experiments were carried out using asymmetric workload. With other model parameters remaining unchanged, the asymmetry is introduced by controlling the movement function, which has different probabilities when forwarding events to different LPs. In the following experiments, when an LP is going to schedule an event, the probability of an event being scheduled at $L P_{i}$ is given by $2.5 \%+(\mathrm{i}-1) / 2$ (note that $\left.\sum_{i=1}^{16}(2.5 \%+(\mathrm{i}-1) / 2)=\mathrm{I}\right)$, i.e., the probability of forwarding an event to $L P_{1}$ through $L P_{16}$ forms an arithmetic sequence with common difference equals to $0.5 \%$. The bigger identification number the LP has, the higher chance the LP receives events.

Figure 6.12 and Figure 6.13 show the results of the experiments running with asymmetric workload on the cluster and in an emulated WAN respectively. Because LPs no longer behave equally under asymmetric workload, the number of rollbacks were measured for all the LPs and the figures plot the data collected at the time GVT ran over 5000. Experiments show that LPs in TTW experienced much higher number of rollbacks under saturated workload in both unicast and multicast environments. For the purpose of a better illustration, the comparison of the rollback curves between TTW and CTW under saturated workload are separated from other workloads in Figure 6.13. The big- 


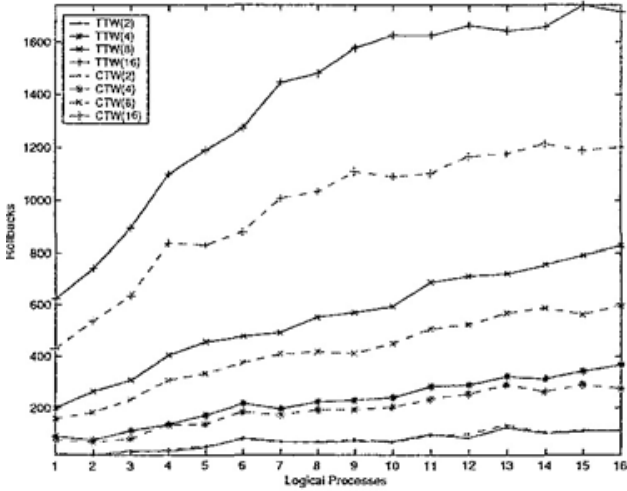

(a) unicast

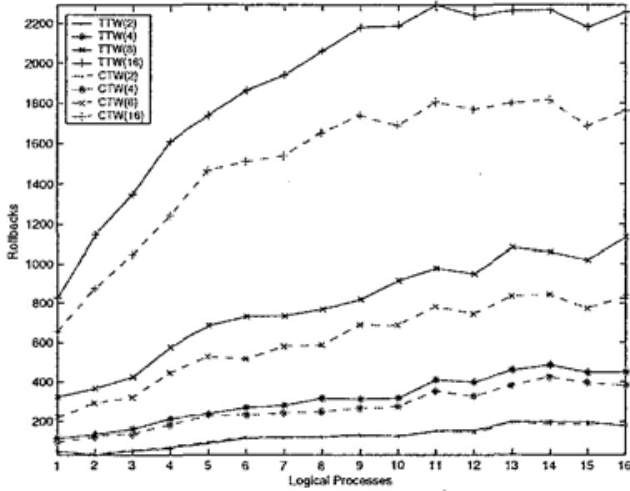

(b) multicast

Figure 6.12: Comparison of Rollback $\left(L P_{i}\right)$ on cluster when GVT=5000 (asymmetric)
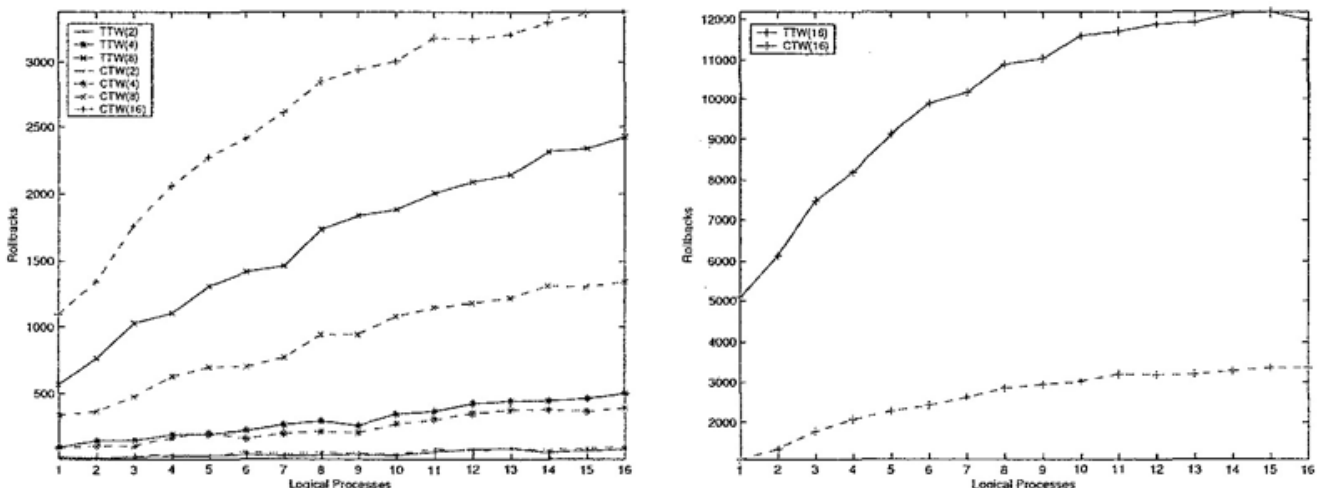

(a) unicast
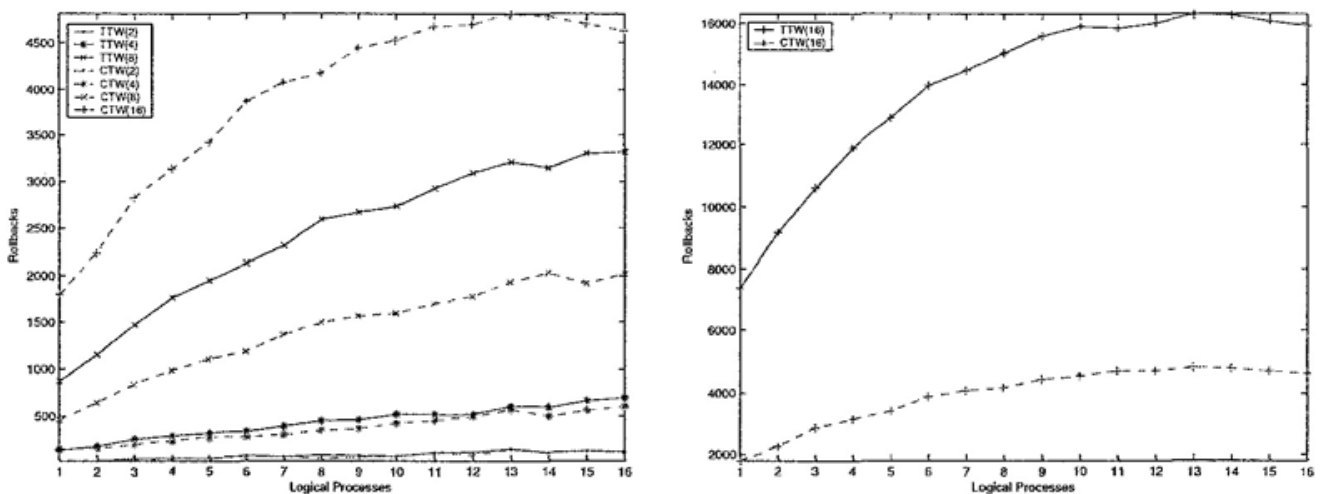

(b) multicast

Figure 6.13: Comparison of $\operatorname{Rollback}\left(L P_{i}\right)$ in emulated WAN when GVT=5000 (asymmetric) 
ger identification number the LP has, the higher workload it may have. So, the results confirm what we have observed previously, i.e., CTW has much better performance than TTW under heavier workload.

Similar observations as those observed in Figure 6.8 and Figure 6.9 are drawn from comparisons among the curves in Figure 6.12 and Figure 6.13: (1) Experiments running on cluster always exhibit much less rollbacks than those running in emulated WAN; (2) Experiments using unicast scheduling incur less rollbacks than using multicast scheduling. Nevertheless, similarity of curves shows that for both executives running with a particular workload, LPs always have similar rollback behaviors using both event scheduling; and (3) CTW shows increasing significance in reduction of rollbacks as workload increases, particularly in the experiments running with saturated workload in the emulated WAN. It is also observed that $L P_{16}$ (the LP processes the most events) does not necessarily experience the most rollbacks. The protruding curves of saturated workload show that LPs with bigger identifiers are more likely throttled.

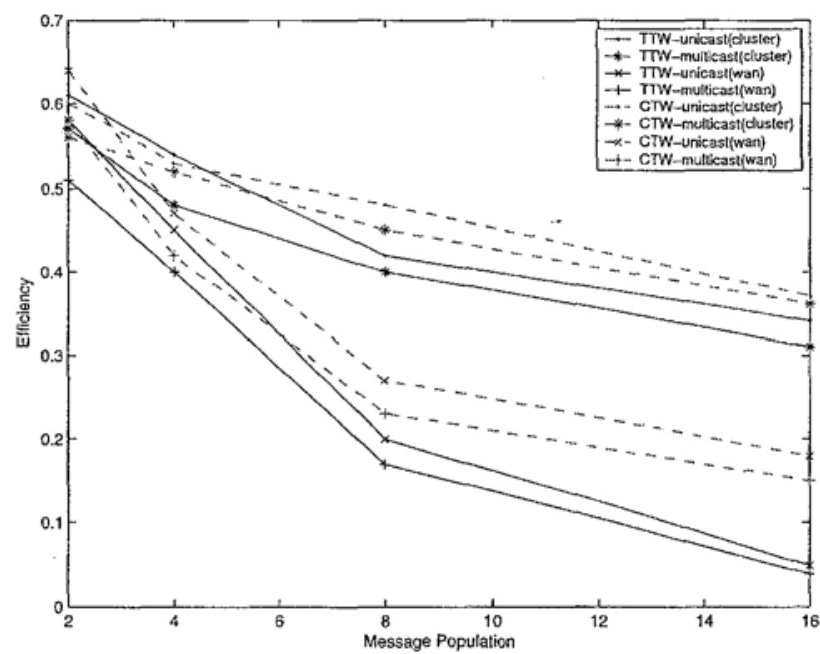

Figure 6.14: Comparison of Efficiency worst $_{(L P)}$ (asymmetric)

Figure 6.14 depicts the lower bound of LPs' efficiency, denoted as $E f$ ficiency $y_{\text {worst }}(L P)$, collected from each experiment. Efficiency $y_{\text {worst }}(L P)$ is essential for the overall perfor- 


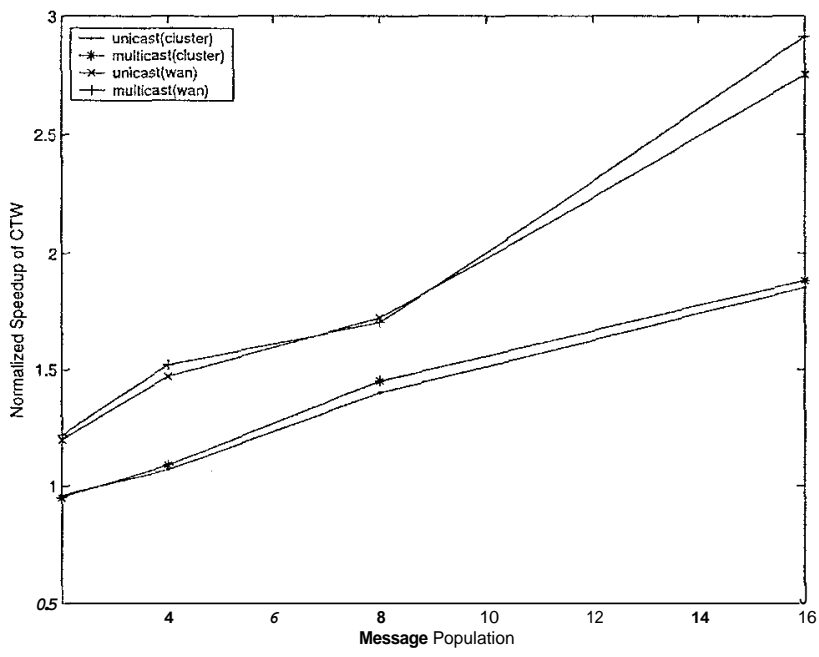

Figure 6.15: Normalized speedup of CTW (asymmetric)

mance of the PHOLD model because LPs are fully interconnected and the efficiency of any LP may subsequently have impact on any of other LPs. It can be seen from Figure 6.14 that CTW surpasses TTW in most cases with higher workload, and moreover, Efficiency $y_{\text {worst }}(L P)$ in CTW is more resilient to the increasing workload (particularly in the emulated WAN environment). This is confirmed by calculated normalized speedup shown in Figure 6.15, in which Norrnalized $(C T W, T T W)$ can be close to 3 in extreme cases.

\subsection{Summary}

In this chapter, we investigated the approach of incorporating the causal order delivery into Time Warp simulations. As discussed in Chapter 2, the causal order is a partial order relation, which means some, but not necessarily all, pairs of messages (events) in the simulation are ordered by the causal order. If a pair of messages (events) cannot be ordered, they are said to be concurrent and can be processed in any sequence. Different from the causal order, Time Warp simulations rely on the concept of simulation time 
and requires that events must be processed in the time-stamp order. The timestamp order can be viewed as a total ordering scheme in which any two events can be ordered upon the two-tuple $\langle i, S T(e)>$, where $i$ is the identification number of the LP which processes the event and $S T(e)$ is the event's time-stamp.

We first show that the causal order may sometimes conflict with the time-stamp order because an LP may not necessarily always schedule external events in non-decreasing time-stamp order. We then investigate the ways to remove the discrepancies. The most intuitive way is to do sending side reordering, where each LP strictly guarantees the consistency between the causal order and the time-stamp order. Once a discrepancy happens, affected outgoing messages are cancelled and resent with adjusted vector time. This approach, however, incurs additional rollbacks and considered less efficient. By studying the way that LPs in traditional Time Warp simulations handle received events, a more efficient way to remove discrepancies at sending side is proposed. According to this way, a discrepancy is allowed to happen but is removed immediately at the receiving LP without being further propagated.

The experiments were carried out using the second approach with symmetric and asymmetric workloads. The performance study shows that CTW effectively reduces the number of rollbacks in all cases, especially where causality violations are prevailing. The consequence of less rollbacks in CTW is that LPs have higher event processing efficiency and speedup, especially in non-FIFO or heavier workload environment.

So far in our discussion and implementation, a simple causal order delivery algorithm [118]is used in COBTW. Compared with Time Warp, external messages in COBTW carry much more information $\left(O\left(N^{2}\right)\right)$ to capture the causal order and guarantee the causal order delivery. This introduces considerable communication overhead in Time Warp simulations. By using the causal order barrier, which captures the immediate causal predecessors of the sent message, instead of the $N \times N \operatorname{SENT}(m)$ matrix, the 
optimal approaches proposed in $[19,112]$ significantly reduce the amount of piggybacked information and, therefore, can be employed to minimize the communication traffic. 


\section{Chapter 7}

\section{Conclusions and Future Work}

The study on the causal order has been carried out for about two decades, during which a significant amount of research work has been done. It proves to be a fundamental relation universally existing among the events in distributed systems and a basis for tackling various issues in distributed systems, e.g., parallelism, determinism and synchronization. It is not exaggerated to say that the causal order has been recognized as the key to study the behaviors of most distributed systems.

After decades of research in parallel and distributed simulation domain, two fundamental issues, namely, consistency issue in distributed virtual environments and performance issue in Time Warp simulations, still prove to be challenging and are open for further study.

In summary, the significance of our research is that we take advantage of valuable research work on the causal order and systematically use the causal order as a powerful tool to explore alternative solutions to the above mentioned two issues. The overall contributions of our research include: (1)The correctness of all the proposed approaches are theoretically proved. (2) Experimental studies show that the proposed approaches to Time Warp simulations achieve performance improvement in popular Time Warp benchmarking models. 
The next two sections further highlight our research achievement and suggest possible future research directions.

\subsection{The Causal Order based Event Ordering in DVEs}

Consistency issues in DVEs are easier to be identified but much more difficult to be tackled. The reason is that solving consistency issues normally interrelates with solving many other issues, e.g., data distribution model, communication model, fidelity requirement and real-time requirement, and to some extent, conflicts with some of them.

Pioneering work in DVEs yields a good taxonomy on consistency study: according to the relation to the time as the state evolves, research on consistency issues can be distinguished from two different domains, namely, the discrete domain and the continuous domain. In recent years, research in the discrete domain has made great progress $[99,100$, 135]. However, research in the continuous domain has just begun. The most challenging issue is the time factor. On one hand, stricter consistency maintenance normally requires more synchronization overhead. On the other hand, real-time requirements are generally application dependent and ideally met by reducing communication and synchronization overheads. This kind of conflict implies that consistency maintenance in any particular continuous domain application has to be tackled as a compromise to its real-time requirement, which results in different consistency levels that might be of interest.

To one extreme, events in DVEs are processed in receiving order, which provides the least consistency maintenance while consumes the least network bandwidth and incurs no synchronization cost. This ordering is mostly used in large scale DVEs in which thousands of entities communicate with each other. To the other extreme, events in DVEs might be totally ordered and processed in the same way as that in analytical simulations. However, neither conservative synchronization nor optimistic synchronization see their fitness in 
most of the applications. As an intermediate ordering scheme, the causal order can achieve a certain degree of consistency whereby causal related messages can be delivered in a consistent way.

The causal order, however, cannot prevent inconsistency caused by false delivery of concurrent messages that have particular semantic relationship, which is desirable to be avoided in some DVEs. Our research in this issue results in the direct-follow relation and the direct-follow order (DFO) delivery proposed in Chapter 3. The DFO is based on the causal order thus has stronger ordering capability. With the DFO, not only messages are delivered in the causal order, but also directly coupled causal messages can be ideally delivered in uninterrupted manner, e.g., the "green” and '(move”messages in the traffic example (see Figure 3.2).

Our study also indicates that the DFO cannot be always fulfilled due to possible conflict with the causal order in some situations. This is because the DFO imposes dependencies among concurrent messages. Together with dependencies introduced by the causal order, a circular dependency may eventually exist among a certain number of messages. This knowledge is heuristic and general to any ordering scheme which is based on the causal order and attempts to order concurrent messages.

We have shown that the conflict can be detected by constructing dependency set for each message, and the DFO is easier to be implemented if direct-follow messages are pre-defined using application specific information. However, implementation of the DFO in the general case is more difficult and additional protocol messages are needed.

Future work in the DFO is more implementation-oriented. Chapter 3 gives a definition of the dependency set of a message. But how this set is practically constructed and how this construction can be done efficiently are yet to be studied. Besides DFO algorithms using application specific information, a more general DFO using protocol messages should be investigated as well. Not limited to the DFO, other consistency lev- 
els are also worthy of study. Other than research on ordering schemes stronger than the causal order, weaker consistency requirements need to be identified and weaker ordering schemes are to be explored in the future.

\subsection{Exploring Causality in Time Warp Simulations}

The purpose of using analytical simulations is to emulate and study the behavior of physical systems. Theoretically, the correctness of the simulation is guaranteed by fully preserving the chronological order in which the physical system evolves. Thus the timestamp order of processing events is regarded as the ultimate rule of any simulation model.

In Time Warp simulations, because LPs advance optimistically, the time-stamp order is ensured by adherence to the local causality constraint. Once a violation is encountered, affected LPs have to roll back to perviously checkpointed states in order to properly recover the computation.

The process of recovery, particularly secondary rollbacks during the process, have been recognized as being complicated and time-consuming due to communication involved among LPs. Moreover, Inefficient rollback mechanism has been realized as the reasons for several performance pitfalls of Time Warp simulations. In our research, we aim to develop more effective rollback mechanism by exploring causalities between events and messages in Time Warp simulations and our efforts have been formulated as two approaches.

In the approach of batch based cancellation, we address the inefficiency of per-event based cancellation scheme that is used in traditional Time Warp simulations. The reason for inefficiency is because simulation time is only a weak clock for causality. It is impossible for simulation time to capture relations between messages in cancellation, which is required for more efficient cancellation schemes. By introducing the state vector into Time Warp simulations, it is shown that the dependance of events and messages is able 
to be captured and identified and therefore the cancellation can be done in optimal way with the cost of at most one primary or secondary rollback at each LP

In the approach of the causal order based Time Warp, we address the inefficiency of excessive number of rollbacks from which LPs could suffer where communication channels are non-FIFO or multicast communication prevail. The idea is to process messages in the causal order rather than in receive order in traditional Time Warp simulations so that messages disordered by channels can be ordered by the causal order delivery protocol. There, however, exist challenges to remove the discrepancies between the causal order and time-stamp order. Our study shows that these discrepancies can be removed at LPs at the time of receiving messages.

Our experiments confirm the merits of proposed approaches in manufacturing and PHOLD models. However, there are still several issues need to be addressed in future study to fully explore their advantages.

In our current research, batch based cancellation scheme is only discussed with the assumption of aggressive cancellation strategy and frequent state saving. Theoretically, it is also applicable in simulations using lazy cancellation or infrequent state saving. Techniques of reducing communication overhead and computing overhead are critical for more efficiency. This can be done by reducing the size of rollback histories carried by messages and rollback history tables locally stored by LPs using specific application information. For example, an LP in a particular simulation only communicates with small amount of LPs, only entries for these LPs are required to be processed and stored.

For a simulation using FIFO communication channels in which each LP always schedules events with non-decreasing time-stamps before it rolls back, it suffices to say that LPs with only one incoming channel will never receive any straggler message and originate any cancellation. Therefore, their rollback behaviors are completely determined by their preceding LPs. The state counters of these LPs can be eliminated from state vector. 
In addition to reducing the number of entries, data structures of rollback histories are also needed to be studied to provide more efficient lookup.

For the causal order based Time Warp (COBTW), current implementation is the simplest but costly. There exist general but more efficient algorithms to guarantee the causal order delivery with respect to reducing communication overheads. It is expected that they have performance gain over current implementation when the number of LPs involved in the causal order delivery increases. The biggest limitation to COBTW, however, is the scalability problem to the number of LPs, which is intrinsic to the causal order delivery algorithm itself. Nevertheless for a large scale simulation, it has been realized that partitioning the simulation into clusters is a key technique to achieve higher performance. The causal order based approach can still find its use in inter-cluster or intra-cluster synchronization. Current research on COBTW is independent to the research on batch based cancellation scheme. COBTW still uses anti-messages to do per-event based cancellation. It is worthy of investigating in the future to combine these two approaches together and see how COBTW benefits from the batch based cancellation. 


\section{Author's Publications}

1. Y. Zeng, W. Cai, and S.J. Turner. Causal order based Time Warp: A tradeoff of optimism. In Proceedings of the 2003 Winter Simulation Conference, pages:855$863,2003$.

2. Y. Zeng, W. Cai, S.J. Turner, S.Zhou, and B. S. Lee. Characterization and delivery of directly coupled causal messages in distributed systems. Future Generation Computer Systems, 20(1):171-178, 2004.

3. Y. Zeng, W. Cai, and S.J. Turner. Batch based cancellation: a rollback optimal cancellation scheme in Time Warp simulations. In Proceedings of the $18^{\text {th }}$ workshop on Parallel and distributed simulation, pages:78-86, 2004.

4. Y. Zeng, W. Cai, and S.J. Turner. Causal order delivery of events in Time Warp simulations. Under preparation for submission.

5. Y. Zeng, W. Cai, and S.J. Turner. Batch based cancellation in Time Warp simulations. Under preparation for submisszon. 


\section{Appendix A}

\section{Sample Code Running on TTW and CTW}

The following two programs (Listl and List2) demonstrate the steps to build simulation program on top of TTW executive and CTW executive respectively. To better present the ideas, the benchmark code of PHOLD used in Chapter 6 are used and only code for two event threads using unicast are shown for simplicity.

Recall the discussion on Figure 4.3, only four classes (PhysicalProcess,LogicalProcess, Message, State) are required to be derived in applications. Moreover, application developers should derive them directly from the ones that provided by the executive in order to run on top of the latter. As shown in List1 for example, PhysicalProcess is derived as TTWPhysicalProcess in TTW, the application using TTW should implement its own PHOLDPhysicalProcess by deriving it from TTWPhysicalProcess.

Listl and List2 are self-explained. Comparison between them shows that the choice of different executive by an application is just a matter of choosing different group of base classes provided by the executives. Thanks to the Time Warp mechanism generalized by ATW, derived executives TTW, BTW and CTW run with common structure, provide uniform interfaces to application but without loss of their specialization. This makes "fair" performance comparison of these executives possible in our experiments. 
// List1: PHOLD running on TTW with two event threads and unicast of messages \#include "twsk.h"

\#include "DataBuffer.h"

\#include "TTWMessage.h"

\#include "TTWPhysicalProcess.h"

\#include "TTWLogicalProcess.h"

\#include "Stopwatch.h"

\#include "Exception.h"

\#define PHOLDMSG TWSK_USERMS

// the type identifier of PHOLDMessage

// PHOLDMessage is the only user-defined message used in PHOLD model

class PHOLDMessage: public TTWMessage \{

public:

PHOLDMessage( ) :TTWMessage( $)$ ( type $=$ PHOLDMSG

$/ /$ default constructor

PHOLDMessage (const PHOLDMessage\& $\mathrm{msg}$ ) \{

\} TTWMessage: : operator $=(\mathrm{msg})$;

vojd serialize(DataBuffer\& buf) const \{ TTWMessage: : serialize(buf)

void deserialize(DataBuffer\& buf) \{ TTWMessage: :deserialize(buf)

\}

$/ /$ set the message type

$/ /$ copy constructor

// no serialization for user-defined data

// serialize base class TTWMessage only

// no deserialization for user-defined data

// deserialize base calss TTWMessage only

\};

// allocator for PHOLDMessage

Message* allocPHOLDMessage() \{

\}

return new PHOLDMessage( $)$

// State of LP

class PHOLDState: public TTWState \{

public:

int count;

PHOLDState(): TTWState(), count(0) \}

PHOLDState (const PHOLDState\& s) \{

TTWState: : operator $=(\mathrm{s})$;

count $=s$. count

State* alloc_state() \{

\}

return new PHOLDState(*this);

子;

// Physical Process in PHOLD model

class PHOLDPhysicalProcess: pubłic TTWPhysicalProcess \{ public:

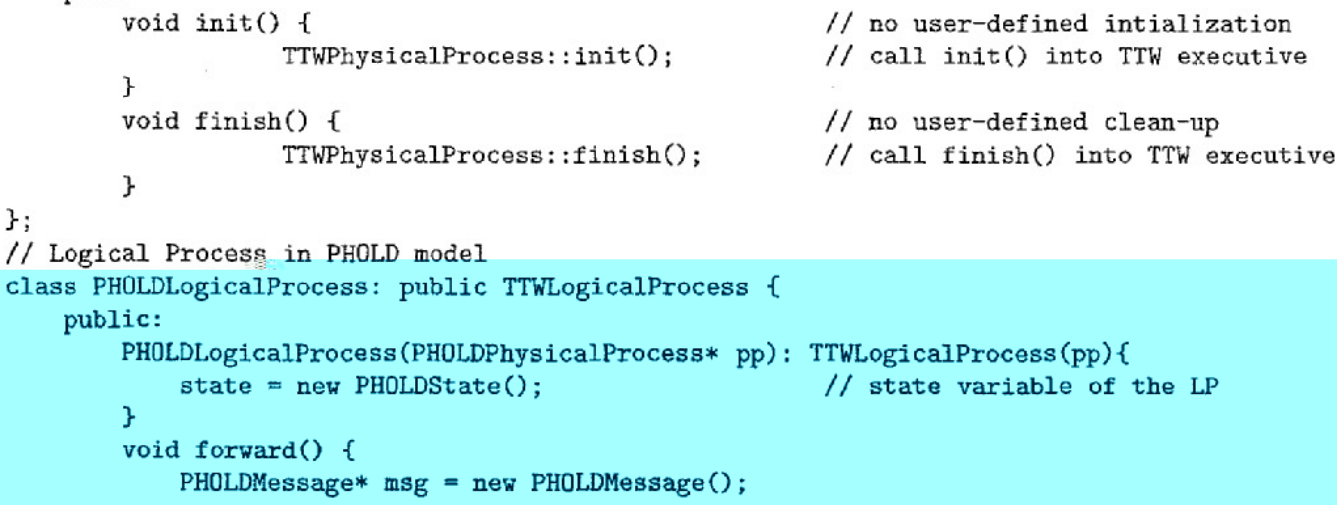

TTWPhysicalProcess: finish(); // call finish() into TTW executive

$/ /$ no user-defined intialization

$/ /$ call init() into TTW executive

$/ /$ no user-defined clean-up

void forward() \{

PHOLDMessage* msg $=$ new PHOLDMessage ();

$/ /$ copy constructor

// allocate state for state saving 


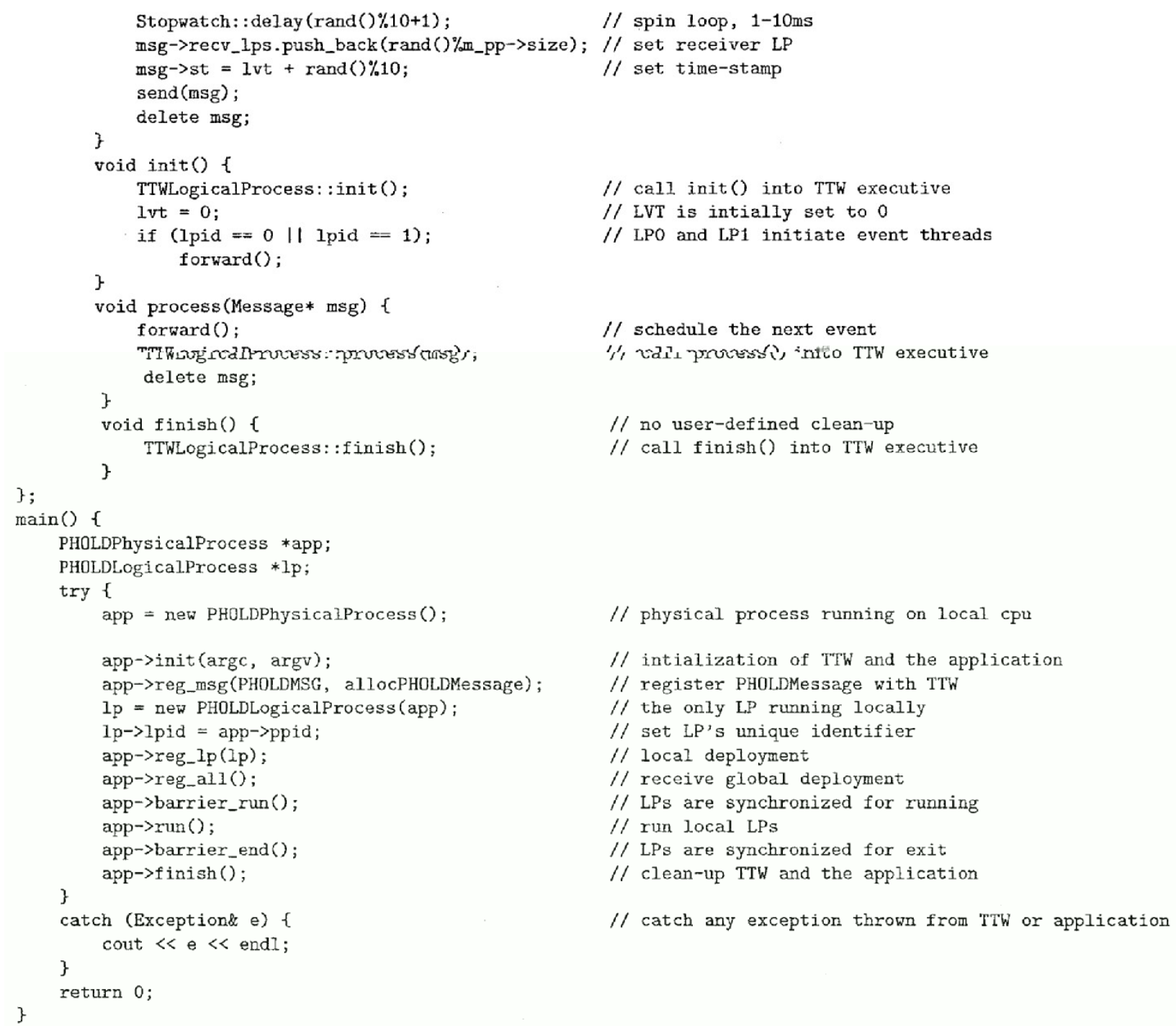




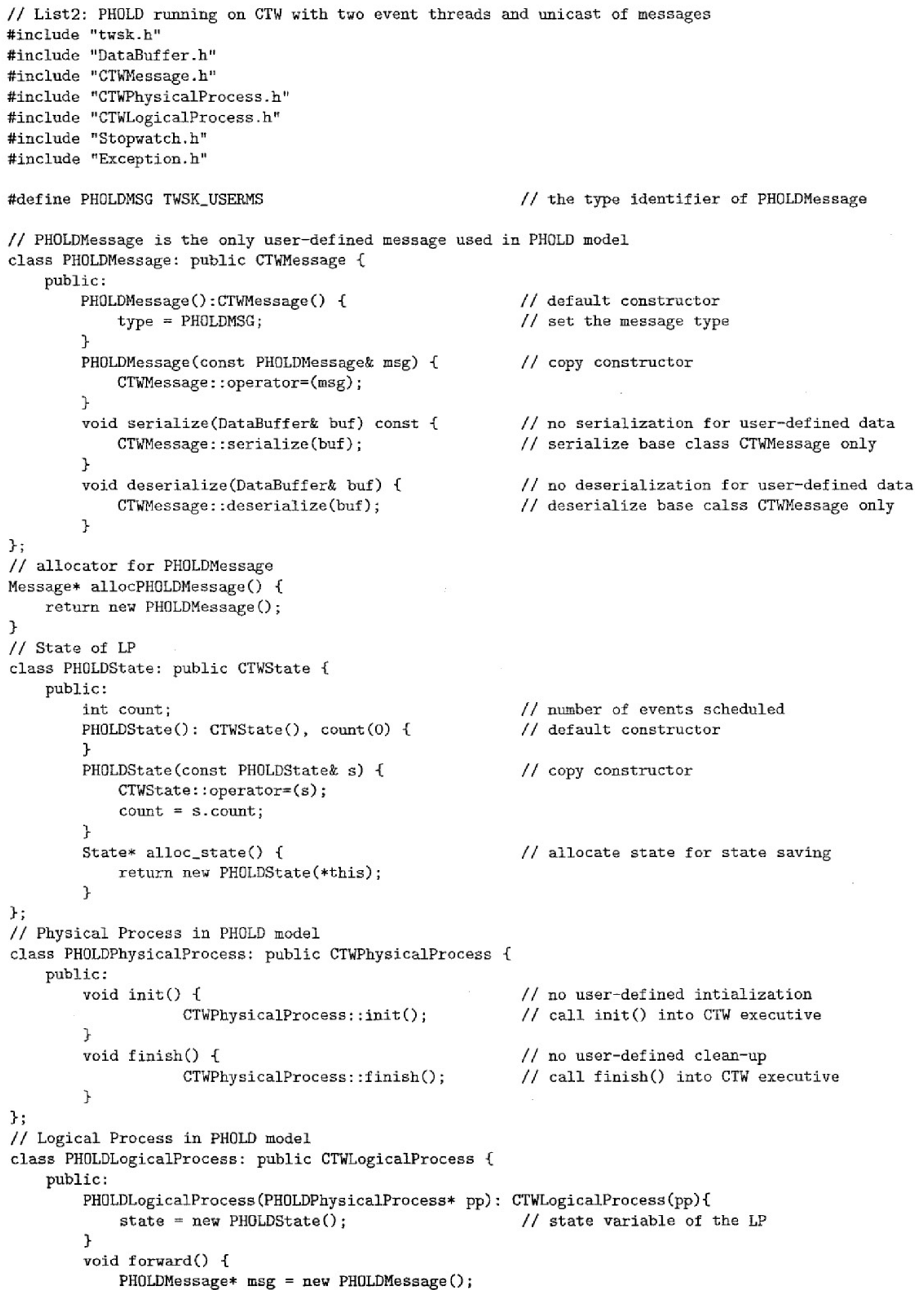




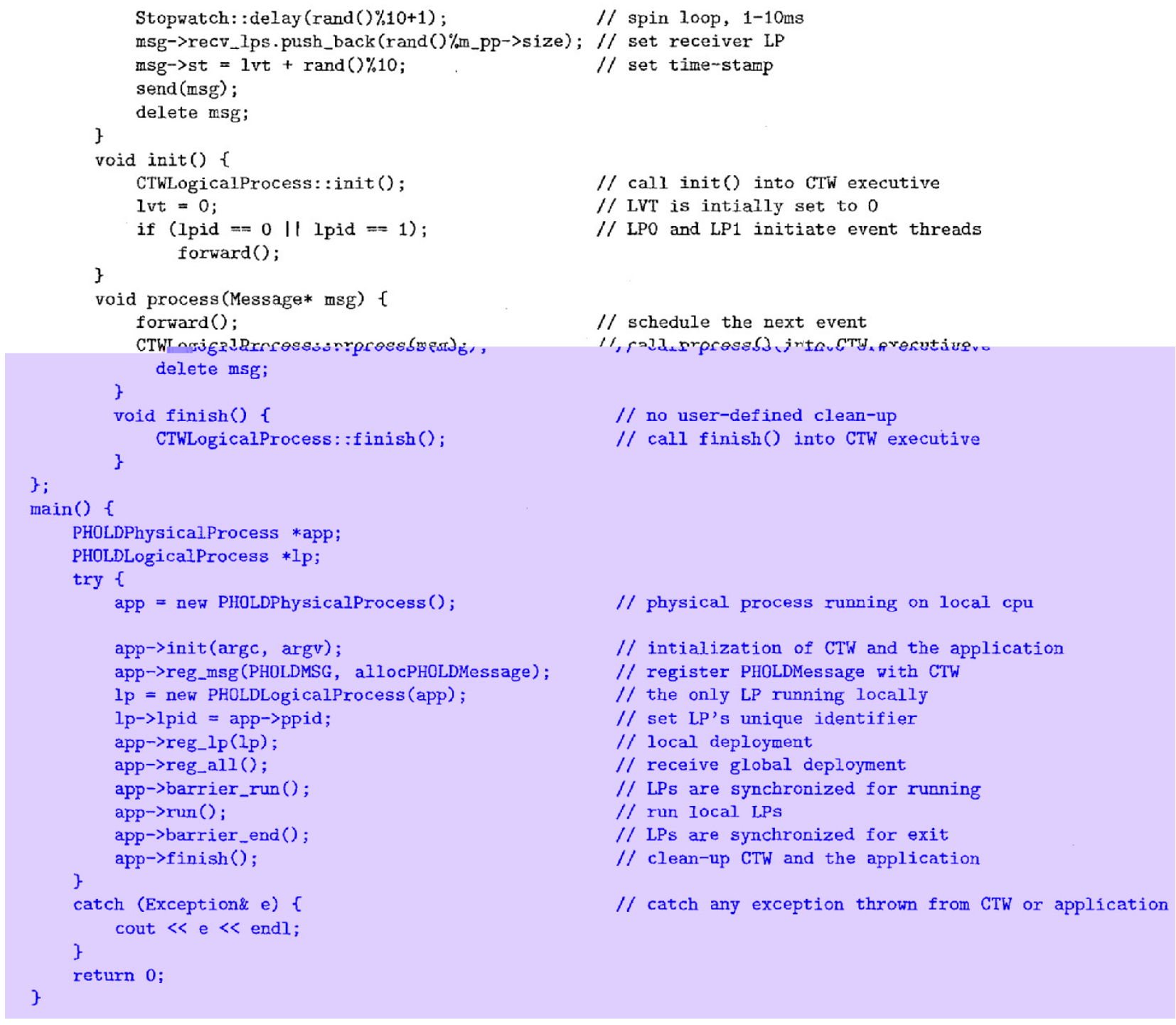




\section{Bibliography}

[1] A. Acharya and B. Badrinath. Recording distributed snapshots based on causal order of message delivery. Information Processing Letters, 44:317-321, 1992.

[2] M. Ahamad, G. Neiger, J. E.Burns, P. W. Hutto, and P. Kohli. Causal memory: Definitions, implementation and programming. Distributed Computing, 9:37-49, 1995.

[3] S. Alagar and S.Venkatesan. An optimal algorithm for distributed snapshots with causal message ordering. Information Processing Letters, 50:311-316, 1994.

[4] D. B. Anderson, J. W. Barrus, J. H. Howard, C. Rich, C. Shen, and R. C. Waters. Building multi-user interactive multimedia environments at MERL. IEEE Multimedia, 2(4):77-82, Winter 1995. Also published as MERL technical report TR 95-17.

[5] R. Ayani. A parallel simulation scheme based on distances between objects. In Proceedings of the SCS Multiconference on Distributed Simulation, pages 113-118, 1989.

[6] R. Ayani and H. Rajaei. Parallel simulation using conservative time window. In Proceedings of the 24th conference on Winter simulation, pages 709-717, 1992. 
[7] H. Bal, A. Plaat, M. Bakker, P. Dozy, and R. Hofman. Optimizing parallel applications for wide-area clusters. In Proceedings of the 12th International Parallel Processing Symposium, pages 784-790, April 1998.

[8] P. Baldy, H. Dicky, R. Medina, M. Morvan, and J. F. Vilarem. Efficient reconstruction of the causal relationship in distributed computations. In Canada-France Conference on Parallel and Distributed Computing, pages 101-113, 1994.

[9] L. Barriga, R. Ronngren, and R. Ayani. Benchmarking parallel simulation algorithms. In Proceedings of the IEEE International Conference on Algorithms and Architectures for Parallel Processing, pages 611-620, 1995.

[lo] B. N. Bershad and M. J. Zekauskas. Midway - shared memory parallel programming with entry consistency for distributed memory multiprocessors. Technical Report CMU-CS-91-170, Carnegie Mellon University, 1991.

[Ill K. Birman. A response to cheriton and skeen's criticism of causal and totally ordered communication. ACM SIGOPS Operating Systems Review, 28(1):1l-21, 1994.

[12] K. Birman and T. A. Joseph. Reliable communication in the presence of failures. A CM Transactions on Computer Systems, 5( 1):47-76, 1987.

[13] K. Birrnan and R. Renesse. Reliable distributed computing with the ISIS toolkit. IEEE Computer Society Press, 1994.

[14] K. Birman, A. Schiper, and P. Stephenson. Lightweight causal and atomic group multicast. ACM Transactions on Computer Systems, 9(3):272-314, August 1991.

[15] J. Briner Jr. Fast parallel simulation of digital systems. In Proceedings of the SCS Multiconference on Advances in Parallel and Distributed Simulation, pages 71-77, 1991. 
[16] W. Broll. Interacting in distributed collaborative VE. Proceedings of VRAIS'95, page 148, 1995.

[17] R. E. Bryant. A switch level model simulator for MOS digital systems. IEEE Transactions on Computers, C-33(2):160-177, 1984.

[18] W. Cai, B. S. Lee, and L. Chen. An auto-adaptive dead reckoning algorithm for distributed interactive simulation. In Proceedings of the 13th workshop on Parallel and distributed simulation, pages 82-89, 1999.

[19] W. Cai, B.S. Lee, and J. Zhou. Causal order delivery in multicast environment: An improved algorithm. Journal of Parallel and Distributed Computing, 62(1), 2002.

[20] W. Cai and S. J. Turner. An algorithm for distributed discrete-event simulation - the 'carrier-null message' approach. In Proceedings of SCS Multiconference on Distributed Simulation, pages 3-8, January 1990.

[21] C. D. Carothers, R. M. Fujimoto, Y. B.Lin, and P. England. Distributed simulation of large scale pcs networks. In Modeling, Analysis, and Simulation of Computer and Telecommunication Systems (MASCOTS' 94) pages 2-11, January.

[22] C. D. Carothers, Y. B. Lin, and R. Fujimoto. Simulating population dependent PCS network models using time warp. In Proceedings of the 27th conference on Winter simulation, pages 555-562, 1995.

[23] K. M. Chandy and L. Lamport. Distributed snapshots: Determining global states of distributed systems. ACM Transactions on Computer Systems, 3(1):63-75, 1985.

[24] K. M. Chandy and 3. Misra. Distributed simulation: A case study in design and verification of distributed programs. IEEE Transactions on Software Engineering, SE5(5):440-452, 1979. 
[25] E(. M. Chandy and 3. Misra. Asynchronous distributed simulation via a sequence of parallel computations. Communications of the ACM, 24(4):198-206, 1981.

[26] E(. M. Chandy, J. Misra, and L. M. Haas. Distributed deadlock detection. ACM Transactions On Computer Systems, 1(2):144-156, May 1983.

[27] B. Charron-Bost. Concerning the size of logical clocks in distributed systems. Information Processing Letters, 39:11-16, July 1991.

[28] D. R. Cheriton and D. Skeen. Understanding the limitations of causally and totally ordered communication. In Proceedings of the 14th ACM symposium on Operating systems principles, pages 44-57, 1993.

[29] M. Chetlur and P. A. Wilsey. Causality representation and cancellation mechanism in time warp simulations. In Proceedings of the 15th workshop on Parallel and distributed simulation, pages 165-172, May 2001.

[30]T. Chiueh. Distributed systems support for networked games. In Proceedings of the 6th Workshop on Hot Topics in Operating Systems, pages 99-104, May 1997.

[31] T. Chiueh, A. Ballman, and P. Pradhan. Distributed system support for networkbased multi-user interactive applications. In Proceedings of 1st Distributed Simulation Symposium, Sept. 1997.

[32] J. G. Cleary and J. J. Tsai. Performance of a conservative simulator of ATM networks. ACM SIGSIM Simulation Digest, 27(1)142-145, 1997.

[33] R. Cooper and K. Marzullo. Consistent detection of global predicates. In ACM/ONR Workshop on Parallel and distributed Debugging, pages 163-173, 1991.

[34] F. Cristian, H. Aghali, R. Strong, and D. Dolev. Atomic broadcast: From simple message diffusion to byzantine agreement. In International Conference on Fault 
Tolerant Computing, pages 200-206, Ann Arbor, MI, USA, 1985. IEEE Computer Society Press.

[35] O. P. Damani, Y. M. Wang, and V. K. Garg. Optimistic distributed simulation based on transitive dependency tracking. In Proceedings of the 11th workshop on Parallel and distributed simulation, pages 90-97, 1997.

[36] S. R. Das and R. M. Fujimoto. An empirical evaluation of performance-memory trade-offs in Time Warp. IEEE Transactions on Parallel and Distributed Systems, 8(2):210-224, 1997.

[37] Defense Advanced Research Projects Agency. Synthetic Theater of War. http://stow98. spawar.navy.mil, 2002.

[38] J. M. S.Dias, R. Galli, A. C. Almeida, C. A. C. Bello, and J. M. Rebordao. mWorld: A multiuser 3D virtual environment. IEEE Computer Gruphics and Applications, 17(2):55-65, March 1997.

[39] P. M. Dickens, P. Heidelberger, and D. NI. Nicol. Parallelized direct execution simulation of message-passing parallel programs. IEEE Transactions on Purullel and Distributed Systems, 7(10):1090-1105,1996.

[40]P. M. Dickens and P. Reynolds. SRADS with local rollback. In Proceedings of the SCS Multiconference on Distributed Simulation, pages 161-164, 1990.

[41] C. Diot and L. Gautier. A distributed architecture for multiplayer interactive applications on the Internet. IEBE Network, 13(4):6-15, July/August 1999.

[42] L. D'Souza, X. Fan, and P. Wilsey. pGVT: An algorithm for accurate GVT estimation. In Proceedings of the 8th Workshop on Parallel and Distributed Simulation, pages 102-109, July 1994. 
[43] M. Dubois, C. Scheurich, and F. Briggs. Memory access buffering in multiprocessors. In 13th Annual International Symposium on Computer Architecture, pages 434-442, 1986.

[44] T. P. Duncan and D. Grazanin. Pre-reckoning algorithm for distributed virtual environments. In Proceedings of the 2003 Winter Simulation Conference, pages 1086-1093.

[45] R. Felderman and L. Kleinrock. Two processor time warp analysis: A unifying approach. Technical Report RS-92-300, USC Information Sciences Institute, 1992.

[46] R. Felderman and L. Kleinrock. Two processor time warp analysis: Capturing the effects of message queueing and rollback / state saving costs. Technical Report RS-93-367, USC Information Sciences Institute, 1992.

[47] A. Ferscha. Handbook of Parallel and Distributed Computing, chapter Parallel and Distributed Simulation of Discrete Event Systems, pages 1003-1041. McGraw-Hill, 1996.

[48] A. Ferscha and G. Chiola. Self-adaptive logical processes: the probabilistic distributed simulation protocol. In Proceedings of the 27th Annual Simulation Symposium, LaJolla, 1994. IEEE Computer Society Press.

1491 C. J. Fidge. Timestamps in message-passing systems that preserve the partial ordering. In Proceedings of the 1Ith Australian Computer Science Conference,pages 56-66, 1988.

[50] C. J. Fidge. Logical time in distributed computing systems. IEEE Computer, 24(8):28-33, August 1991.

[51] M. J. Fischer and A. Michael. Sacrificing serializability to attain high availability of data. In Symposium on Principles of Database Systems, pages 70-75, 1982. 
[52] P. A. Fishwick. Simulation Model Design and Execution: Building Digital Worlds. McGraw-Hill, New York, USA, 1994.

[53] MPI Forum. The Message Passing Interface (MPI) standard. http://wwwunix.mcs.anl.gov/mpi/ .

[54] J. Fowler and W. Zwaenepoel. Causal distributed breakpoints. In Proceedings of the 10th International Conference on Distributed Computing Systems (ICDCS), pages 134-141, Washington, DC, 1990. IEEE Computer Society.

[55] S. Franks, F. Gomes, B. Unger, and J. Cleary. State saving for interactive optimistic simulation. In Proceedings of the 11th Workshop on Parallel and Distributed Simulations, pages 72-79, 1997.

[56]R. M. Fujimoto. Performance measurements of distributed simulaton strategies. Transactions of the Society for Computer Simulation, 6(2):89-132, 1989.

[57] R. M. Fujimoto. Time warp on a shared memory multiprocessor. Transactions of the Society for Computer Simulation International, 6(3):211-239, 1989.

[58] R. M. Fujimoto. Performance of time warp under synthetic workloads. In Proceedings of the SCS Multiconference on Distributed Simulation, volume 22, pages 23-28, 1990.

[59] R. M. Fujimoto. Parallel and Distributed Simulation Systems. Wiley Book Series on Parallel and Distributed Computing. Wiley, New York, NU 10158, USA, 1999.

[60] A. Gafni. Rollback mechanisms for optimistic distributed simulation systems. In Proceedings of the SCS Multiconference on Distributed Simulation, pages 61-67, 1988. 
[61] C. Greenhalgh and S. Benford. A distributed virtual reality system incorporating spatial trading. In Proceedings of the 15th International Conference on Distributed Computing Systems (DCS'95), pages 27-34, May 1995. IEEE Computer Society Press.

[62] C. Greenhalgh and S. Benford. A multicast network architecture for large scale collaborative virtual environments. In Multimedia Applications, Services and Techniques - ECMAST'S'7, Proceedings of the Second European Conference, volume 1242 of Lecture Notes in Computer Science, pages 113-128, May 1997.

[63] R. Guerraoui and A. Schiper. Total order multicast to multiple groups. In Proceedings of the 17th International Conference on Distributed Computing Systems (ICDCS-17), pages 578-585, 1997.

[64] A. Gupta, I. F. Akyildiz, and R. M. Fujimoto. Performance analysis of time warp with multiple homogeneous processors. IEEE Transactions on Software Engineering, 17(10):1013-1027,1991.

[65] O. Hagsand. Interactive multiusers VEs in the DIVE system. IEEE Multimedia, 3(1):30-39, 1996.

[66] NI. P. Herlihy and J. M. Wing. Linearizability: a correctness condition for concurrent objects. ACM Transactions on Programming Languages and Systems, 12(3):463-492,1990.

[67] H. W. Holbrook, S.K. Singhal, and D. R. Cheriton. Log-based receiver-reliable multicast for distributed interactive simulation. In Proceedings of SIGCOMM'95, volume 25(4), pages 328-341, October 1995.

[68] IEEE Standard 1278.1-1995 for Distributed Interactive Simulation-Application Protocols., 1995. 
[69] IEEE Standard 1278.2-1995 for Distributed Interactive SimulationCommunication Services and Profiles., 1995.

[70] IEEE Standard 1516 for High Level Architecture, September 2000.

[71] D. R. Jefferson. Virtual time. ACM Transactions on Programming Languages and Systems, 7(3):404-425, July 1985.

[72] K. L. Kapp, T. C. Hartrum, and T. S.Wailes. An improved cost function for static partitioning of parallel circuit simulations using a conservative synchronization protocol. In Proceedings of the 9th workshop on Parallel and distributed simulation, pages $78-85,1995$.

[73] P. Kearns and B. Koodalattupuram. Immediate ordered service in distributed systems. In Proceedings of the 9th International Conference on Distributed Computing Systems (ICDCS), page 120, Washington, DC, 1989. IEEE Computer Society.

[74]H. K. Kim and J. Jean. Concurrency preserving partitioning (CCP) for parallel logic simulation. In Proceedings of the 10th workshop on Parallel and distributed simulation, pages 98-105, 1996.

[75] P. Konas and P.-C. Yew. Synchronous parallel discrete event simulation on sharedmemory multiprocessors. In Proceedings of the 6th Workshop on Parallel and Distributed Simulation, pages 12-21, 1992.

[76] P. Konas and P.-CYew. Improved parallel architectural simulations on sharedmemory multiprocessors. In Proceedings of the 8th workshop on Parallel and distributed simulation, pages 156-159, 1994.

[77]D. Krishnaswamy, P. Banerjee, E. M. Rudnick, and J. H. Patel. Asynchronous parallel algorithms for test set partitioned fault simulation. In Proceedings of the 11th workshop on Parallel and distributed simulation, pages 30-37, 1997. 
[78] A. D. Kshemkalyani and M. Singhal. Necessary and sufficient conditions on information for causal message ordering and their optimal implementation. Distributed Computing, 11(2):91-111, 1998.

[79] K. Kumaran, B. Lubachevsky, and A. Elwalid. Massively parallel simulations of ATM systems. AGM SIGSIM Simulation Digest, 26(1):39-46, 1996.

[80] L. Lamport. Time,clocks, and the ordering of events in a distributed system. Communications of the AGM, 21(7):558-565, July 1978.

[81] L. Lamport. How to make a multiprocessor computer that correctly executes multiprocess programs. IEEE Transactions on Computers, c-28(9):690-691, 1979.

[82] A. M. Law and D. Kelton. Simulation Modelling and Analysis. McGraw-Hill, New York, USA, 1991.

[83] J. Leigh, A. Johnson, C. Vasilakis, and T. DeFanti. Multi-perspective collaborative design in persistent networked virtual environments. In Proceedings of IEEE Virtual Reality Annual International Symposium '96, page 253, April 1996.

and [84]Chu-Cheow Lim, Yoke-Hean Low, Wentong Cai, Wen- Wentong C. M 
[87]Y. B. Lin, B. R. Preiss, W. M. Loucks, and E. D. Lazowska. Selecting the checkpoint interval in time warp simulations. In Proceedings of the 7th Workshop on Parallel and Distributed Simulations, pages 3-10, 1993.

[88] R. J. Lipton and D. W. Mizell. Time warp vs. chandy-misra: A worst-case comparison. In Proceedings of the SCS Multiconference on Distributed Simulation, pages 137-143, 1990.

[89] B. D. Lubachevsky. Efficient distributed event-driven simulations of multiple-loop networks. Communications of the ACM, 32(1):111-123, 1989.

[90] M. R. Macedonia, D. P. Brutzman, M. J. Zyda, D. R. Pratt, P. T. Barham, 3. Falby, and 3. Locke. NPSNET: A multi-player 3D virtual environment over the Internet. In Proceedings of the 1995 Symposium on Interactive 30 Graphics, pages 9-12, April 1995.

[91] M. R. Macedonia and M. Zyda. A taxonomy for networked virtual environments. IEEE Multimedia, 4(1):48-56, 1997.

[92] M. R. Macedonia, M. J. Zyda, D. R. Pratt, P. T. Barham, and S.Zeswitz. NPSNET: A network software architecture for large scale virtual environments. Presence, 3(4):265-287, Fall 1994.

(931 V. Madisetti, J. Walrand, and D. Messerschmitt. WOLF: A rollback algorithm for optimistic distributed simulation systems. In Proceedings of the 20th conference on Winter simulation, pages 296-305, San Diego, California, 1988.

[94] K. Marzullo and G. Neiger. Detection of global state predicates. In Proceedings of the 5th Workshop on Distributed Algorithms (WDA G-91), pages 254-272, Delphi, Greece, 1991. 
[95] M. MatijaSeviC. A review of networked multi-user virtual environments. Technical Report TR-97-8-1, The University of Southwestern Louisiana, 1997.

[96] F. Mattern. Algorithms for distributed termination detection. Distributed Computing, 2(3):161-175, 1987.

[97] F. Mattern. Virtual time and global states of distributed systems. In M.Cosnard et. al., editor, Parallel and Distributed Algorithms: proceedings of the International Workshop on Parallel and Distributed Algorithms, pages 215-226. Elsevier Science Publishers B. V., 1989.

[98] F. Mattern. Efficient algorithms for distributed snapshots and global virtual time approximation. Journal of Parallel and Distributed Computing, 18(4):423-434, 1993.

[99] M. Mauve. Consistency in replicated continuous interactive media. In Proceeding of the ACM 2000 Conference on Computer supported cooperative work,pages 181-190, 2000.

[loo] M. Mauve, J. Vogel, V. Hilt, and W. Effelsberg. Local-lag and timewarp: Providing consistency for replicated continuous applications. IEEE Transactions on Multimedia, 6(1):47-57, 2004.

[101] S. Meldal, S. Sankar, and J. Vera. Exploiting locality in maintaining potential causality. In Symposium on Principles of Distributed Computing, pages 231-239, 1991.

[loa] D. Miller and J. Thorpe. SIMNET: The advent of simulator networking. In Proceedings of the IEEE, volume 83(8), pages 1114-1123, August 1995.

[lo31 J. Misra. Distributed discrete-event simulation. ACM Computing Sermeys, 18(1):39_ 65, 1986. 
[104] A. Mostefaoui and M. Raynal. Causal multicasts in overlapping groups : towards a low cost approach. In Proceedings of the 4th Workshop on Future Trends of Distributed Computing Systems, pages 136-142, September 1993.

[lo51 D. M. Nicol. Noncommittal barrier synchronization. Parallel Computing, (21):529549, 1995.

[106] D. M. Nicol and X. Liu. The Dark Side of Risk (What your mother never told you about Time Warp). Technical Report TR96-298, Dartmouth College, 1996.

11071 M. Nielsen, G. Plotkin, and G. Winskel. Petri nets, event structures and domains, part I. Theoretical Computer Science, 13(1):85-108, January 1980.

El081 Parallel and Distributed Simulation Systems. http://www.cc.gatech.edu/classes/ AY2002/cs700 1fall/Notes/3.

[lo91 J. K. Peacock, J. W. Wong, and E. G. Manning. Distributed simulation using a network of processors. Computer Networks, 3(1):44-56, 1979.

[110] S.Powers, M. Hinds, and J. Morphett. DEE: An architecture for distributed virtual environment gaming. Distributed Systems Engineering, 5(3): 107-117, 1998.

[111] A. Prakash and R. Subramanian. Filter: An algorithm for reducing cascaded rollbacks in optimistic distributed simulations. In Proceedings of the 24th annual symposium on Simulation, pages 123-132, 1991.

[112] R. Prakash, M. Raynal, and M. Singhal. An adaptive causal ordering algorithm suited to mobile computing environments. Journal of Parallel and Distributed Computing, 41:190-204, March 1997. 
[113] V. R. Pratt. Arithmetic + logic + geometry = concurrency. In Proceedings of the First Latin American Symposium on Theoretical Informatics, volume 583, pages 430-447, Sao Paulo, Brazil, 1992. Springer-Verlag.

[114]B. R. Preiss, W. M. Loucks, I. D. MacIntyre, and J. A. Field. Null message cancellation in conservative distributed simulation. In Proceedings of 1991 Workshop on Parallel and Distributed Simulation, pages 33-38, January 1991.

[115] M. Presley, M. Ebling, F. Wieland, and D. R. Jefferson. Benchmarking the time warp operating system with a computer network simulation. In Proceedings of the SCS Multiconference on Distributed Simulation, volume 21, pages 8-13, March 1989.

[116] J. M. Pullen and D. C. Wood. Networking technology and DIS. In Proceedings of the IEEE, volume 83(8), pages 1156-1167, August 1995.

[117] H. Rajaei, R. Ayani, and L. E. Thorelli. The local time warp approach to parallel simulation. ACM SIGSIM Simulation Digest, 23(1).

[118] M. Raynal, A. Schiper, and S.Toueg. The causal ordering abstraction and a simple way to implement it. Information Processing Letters, 39(6):343-350, September 1991.

[119] R. Reddy and R. Garrett. Future technology challenges in distributed interactive simulation. In Proceedings of the IEEE, volume 83(8), pages 1188-1195, August 1995.

[120] R. L. Reiher, R. M. Fujimoto, S.Bellenot, and D. R. Jefferson. Cancellation strategies in optimistic execution systems. In Proceedings of the SCS Multiconference on Distributed Simulation, pages 112-121, 1990. 
[121] L. Rodrigues and P. Verissimo. Causal separators for large-scale multicast communication. In International Conference on Distributed Computing Systems, pages 83-91, 1995.

[122] R. Ronngren and M. Liljenstam. On event ordering in parallel discrete event simulation. In Proceedings of the 13th workshop on Parallel and distributed simulation, pages 38-45, May 1999.

[123] K. Saar. VIRTUS: a collaborative multi-user platform. In Proceedings of the 4th symposium on Virtual reality modeling language, pages 141-152, 1999.

[1241 B. Samadi. Distributed Simulation algorithms and performance analysis. PhD thesis, University of California, Los Angeles, 1985.

[125] A. Schiper, J. Eggli, and A. Sandoz. A new algorithm to implement causal ordering. Proceedings of the 3rd International Workshop on Distributed Algorithms, LNCS Vol. 392:219-232, 1989.

[126] R. Schwarz and F. Mattern. Detecting causal relationships in distributed computations: In search of the holy grail. Distributed Computing, 7(3):149-174, 1994.

[127] M. Singhal, , and A. Kshemkalyani. An efficient implementation of vector clocks. Information Processing Letters, 43:47-52, August 1992.

[128] S.K. Singhal. Effective Remote Modeling in Large-scale Distributed Simulation and Visualization Environments. PhD thesis, Stanford University, August 1996.

[129] J. Smed, T. Kaukoranta, and H. Hakonen. A review on networking and multiplayer computer games. Technical Report 454, Turku Centre for Computer Science, April 2002. 
[130] L. M. Sokol and B. K. Stucky. MTW: Experimental results for a constrained optimistic scheduling paradigm. In Proceedings of the SCS Multiconference on Distributed Simulation, pages 169-173, 1990.

[131] J. Steinman. Breathing time warp. In Proceedings of the 7th Workshop on Parallel and Distributed Simulation, pages 109-118, 1993.

[132] R. E. Strom and S. Yemini. Optimistic recovery in distributed systems. ACM Transactions on Computer Systems, 3(3):204-226, 1985.

[133] C. Sun. Undo as concurrent inverse in group editors. ACM Transactions on Computer-Human Interaction, 9(4), 2002.

[134] C. Sun and W. Cai. Capturing causality by compressed vector clock in real-time group editors. In Proceedings of 16th Annual International Parallel and Distributed Processing Symposium (IPDPS'ZUUZ), Fort Lauderdale, Florida, USA, April 2002.

[135] C. Sun and D. Chen. Consistency maintenance in real-time collaborative graphics editing systems. A CM Transactions on Computer-Human Interaction, 9(1), 2002.

[136] C. Sun, X. Jia, Y. Zhang, Y. Yang, and D. Chen. Achieving convergence, causality preservation, and intention preservation in real-time cooperative editing systems. ACM Transactions on Cornputer-Human Interaction, 5( 1):63-108, 1998.

[137] C. Sun, Y.Yang, Y. Zhang, and D. Chen. Distributed concurrency control in realtime cooperative editing systems. In Asian Computing Science Conference, pages 84-95, 1996.

[138] S. C. Tay and Y. M. Teo. Performance optimization of throttled time-warp simulation. In Annual Simulation Symposium 2001, pages 211-218, 2001. 
[139] S.C. Tay, Y. M. Teo, and S.T. Kong. Speculative parallel simulation with an adaptive throttle scheme. In Proceedings of 11th Workshop on Parallel and Distributed Simulation, pages 116-123, 1997.

[140] Y. M. Teo and S.C. Tay. Performance and granularity control in the spades parallel simulation system. In 1999 International Symposium on Parallel Architectures, Algorithms and Networks (ISPAN '99), pages 94-99, 1999.

[141] A. I. Tomlinson and V. E(. Garg. An algorithm for minimally latent global virtual time. In Proceedings of the 7th Workshop on Parallel and Distributed Simulation, pages 35-42, 1993.

[142] F. J. Torres-Rojas and M. Ahamad. Plausible clocks: Constant size logical clocks for distributed systems. Distributed Computing, 12:179-195, 1999.

[143] S.J. Turner and M. Q. Xu. Performance evaluation of the bounded time warp algorithm. In Proceedings of the 6th Workshop on Parallel and Distributed Simulation, pages 117-126, 1992.

[144] E(. Venkatesh, T. Radhakrishnan, and H. F. Li. Discrete event simulation in a distributed system. In IEEE COMPSAC, pages 123-129, 1986.

11451 C. Ware and R. Balakrishnan. Reaching for objects in VR displays: lag and frame rate. ACM Transactions on Computer-Human Interaction, 1(4):331-356, 1994.

[146] D. West. Optimizing time warp: Lazy rollback and lazy re-evaluation. Master's thesis, University of Calgary, Calgary, Alberta, 1988.

[147] F. Wieland, E. Blair, and T. Zukas. Parallel discrete-event simulation (PDES): a case study in design, development, and performance using SPEEDES. In Proceedings of the 9th Workshop on Parallel and Distributed Simulation, pages 103-110, 1995. 
[148] A. L. Wilson and R. M. Weatherly. The aggregate level simulation protocol: an evolving system. In Proceedings of the 26th conference on Winter simulation, pages 781-787,1994.

[149] G. Winskel. An introduction to event structures. Lecture Notes in Computer Science: Linear Time, Branching Time and Partial Order in Logics and Models for Concurrency, 354:364-397, 1989.

[150] P. Wonnacott and D. Bruce. The APOSTLE simulation language: granularity control and performance data. In Proceedings of the ZUth Workshop on Parallel and Distributed Simulation, pages 114-123, 1996.

[151] J. Zhang and C. Tropper. The dependence list in time warp. In Proceedings of the 15th Workshop on Parallel and Distributed Simulation, pages 35-45, 2001.

[152] S.Zhou, W. Cai, B.S.Lee, and S.J. Turner. Consistency in distributed interactive applications. In Proceedings of European Simulation Interoperability Workshop 2001 (Euro-SIW 2001), UK London, June 2001.

[153] S. Zhou, W. Cai, B. S. Lee, and S. J. Turner. Time-space consistency in largescale distributed virtual environ. ACM Transactions on Modeling and Computer Simulation (TOMACS), 14(1):31-47, 2004.

[154] S.Zhou, W. Cai, S.J. Turner, and B. S.Lee. Critical causality in distributed virtual environments. In Proceedings of the 16th Workshop on Parallel and Distributed Simulation, pages 53-59, 2002. 